\title{
Children's Behavioral Agency within Families in the Context of Migration: A Systematic Review
}

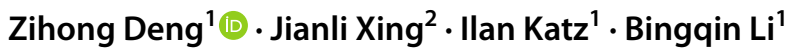

Received: 29 August 2021 / Accepted: 23 September 2021 / Published online: 6 October 2021

(c) The Author(s), under exclusive licence to Springer Nature Switzerland AG 2021

\begin{abstract}
Migration may lead to changing power dynamics between parents and children in families. Children may change their behavior in order to exercise agency to respond to migration of family members or themselves. This systematic review seeks to understand how children exercise agency within families in the context of migration. The authors searched ten databases to collect English-written articles published in academic journals in or after 2010. The studies were coded to generate a quality indicator. 65 Articles with moderate and strong quality were included in this review, including 41 qualitative studies, 16 quantitative studies, and 8 mixed-methods studies. Children and adolescents with demographically and culturally diverse backgrounds were analyzed in these studies. The systematic review shows that children have different levels of behavioral agency in the migration decision-making process; they also exercise agency in different aspects of family life. For example, left-behind children exercise agency in care provision and information nondisclosure, and migrant children in media and language brokering. Children's behavioral agency is place-specific. Adults working with children need to pay more attention to children's behavioral agency in order to support children's healthy development and facilitate their adaptation in the context of migration.
\end{abstract}

Keywords Systematic review $\cdot$ Children's agency $\cdot$ Family $\cdot$ Household $\cdot$ Migration

\section{Introduction}

Children ${ }^{1}$ are aware of their experiences as social actors. Therefore, exercising agency is important to their well-being (Fattore, Mason, \& Watson, 2009). This is consistent with the belief that children should not just be treated as subjects of parents' decisions, and research should give children a voice when analyzing family decisions (Hardman, 2001). Some researchers have focused on the child's perspectives to better understand their experience and feelings around decision-making (Kämpfe \& Westphal, 2016). Migration affects children's life significantly and is an example of an important family decision for large numbers of families. There is a growing body of research examining children's experiences

Zihong Deng

zihong.deng@unsw.edu.au

1 Social Policy Research Centre, University of New South Wales, Sydney, NSW 2052, Australia

2 Department of Social Work and Social Administration, The University of Hong Kong, Pokfulam, Hong Kong SAR, China and whether they can demonstrate agency as families decide to migrate. These studies are based in different locational, social or cultural contexts and use different methodologies. Not having a systematic review to map out the field and summarize the existing research findings makes it difficult for researchers to confidently pinpoint new research directions. This study aims to fill in this gap by conducting a systematic review on children's behavioral agency within their families in the context of migration.

\section{The Concept of Children's Agency and Existing Systematic Reviews}

Agents are defined as "individuals as actors with the ability to make sense of the environment, initiate change, and make choices" (Kuczynski, 2003, p. 9). The concept of agency contains three aspects in social science literature: (1) autonomy, which is the motivational aspect of agency, including self-determination and self-preservation; (2)

\footnotetext{
${ }^{1}$ Children in this review means human offspring not yet reached adulthood which includes young children and adolescents $(<18$ years old).
} 
construction, which is the cognitive dimension of agency, which refers to the capacity to make sense of experiences and create new meanings; and (3) action, which is the behavioral aspect of agency (Kuczynski, 2003). Children's agency can be exercised in different ways, and agentive acts may involve resistance to, complicity with, accommodation to, or reinforcement of status quo (Ahearn, 1999). For example, a single child in a multi-generational family could exercise agency "in sometimes subtle and creative ways, in overt resistance that exploited weaknesses in each of their different relationships, in behavioral compliance accompanied by private rejection of parental messages, in creative attempts at evasion and delay, and in strategically using relationships with some adults to offset the influence of others" (Goh \& Kuczynski, 2009, p. 525). "Sense of agency" refers to "feeling like an agent." "Exercise of agency" refers to "being an agent" or agentic behavior. Sense of agency and exercise of agency may not be consistent with each other (Cummings $\&$ Schermerhorn, 2003). The sense of agency is subjective and may not translate into actions, e.g. children making decisions themselves or making parents take children's needs into account. Although the concept of agency contains different aspects, this systematic review focuses on "the behavioral aspect of agency", i.e. exercising agency or performing agentic behavior. The review examines how children exercise agency in relation to the family's decision-making in the process of family migration. Children's actions may be the outcomes of the power dynamics in their households and can further influence family power relations (Kuczynski, 2003).

The existing literature has reviewed children's agency in different contexts. One progress report discussed children's resistance and resourcefulness in the face of threats to their security, respect, and livelihoods (Jeffrey, 2011). This provides a reference to understand the agency of children in the context of migration. Another review of key quantitative and qualitative research finds that children actively support their mothers and encourage them to make good decisions when facing domestic violence (Katz, 2015). However, that review is not related to migration. One Chinese article has also reviewed children's agency in different studies and discussed the relationship between agency, structure, and culture (Xiao \& Yuan, 2019). These three reviews focused on children's agency but did not report that they used systematic methods to search and screen articles and did not focus on children's agency in the context of migration. Children can exercise their agency in different domains, such as households, peer relationships, schools, communities, service organizations, and political activities and movements. Children's families greatly influence their development and well-being, and power dynamics within households may change during and after migration. Therefore, this study focuses on children's agency within their households.
The content of children's agency can vary as children use different concepts of self-responsibility, e.g. some children associated agency with adaptation, conformity, and selfoptimization, and had strong self-efficacy beliefs, while others associated children's agency with their own preferences, such as fun and enjoyment, or freedom from taking responsibility for their own actions (Kämpfe \& Westphal, 2016). Children's agency also shows cultural differences. In cultures stressing interdependence, individuals may be more collectively agentic, while in cultures stressing independence, individuals may be more personally agentic (Hernandez \& Iyengar, 2001).

The impacts of children exercising agency are not always positive. Sometimes their actions may go against socially approved goals and social norms (Bordonaro, 2012; Sutterlüty \& Tisdall, 2019), such as children's agency in domestic labor in Haiti, and such agency may be considered to be unwelcome due to the victim image of child labor (Hoffman, 2010). Children's agency may be also against their own interests. For example, they might be happy just playing computer games frequently, but this may negatively influence their health and well-being. In summary, children's agency is not homogeneous, and differs according to individual preference, specific circumstances and cultural variations, and agency may affect the children positively or negatively.

\section{Children's Agency in the Context of Migration}

Children can migrate independently or with their parents or stay behind in their hometown when their parents migrate. Data shows $14.6 \%$ of the international migrant stock in the world in 2020 were young migrants aged 19 years and under (UNDESA, 2020), and a large number of children were also involved in domestic migration. Parents and children living in different contexts: parents and children living in new environments in the destination of migration, children living alone in the migration destination, or children living in their hometowns without their parents. These changes in circumstances would affect the way parents and children interact. This review covers children in these different circumstances. The behavioral agency to be studied includes those behaviors related to decisions relevant to their daily lives when living in families living with migration and/or exercising agency when parents live separately (Asis, 2006; Huijsmans, 2011; Orellana et al., 2001). Children, whether involved in migration or not, have a process of agency development; however, migration and the associated issues may complicate this process. Previous studies have identified certain activities as exercising agency in the context of migration, however more studies need to be screened systematically to have a comprehensive understanding of children's various activities to exercise agency. 


\section{Current Study}

This systematic review examines the existing research on children's agency in the context of migration, including both domestic and international migration. The main question is: How do children exercise agency in the family setting in the context of migration? The sub-questions include: (1) How do children exercise agency in the family with regard to decisions in the process of migration? (2) How do children exercise agency in their daily life during/after migration? (3) How do researchers measure children's agency in family in the context of migration? As there is not a mature scale or questionnaire to measure children's strategies of exercising agency in the context of migration, the third sub-question is included to help develop a scale or questionnaire for future studies. Additionally, the influence of migration on child agency is summarized to show the context of migration though it is not a direct sub-question.

\section{Methods}

This systematic review follows the guidelines of Preferred Reporting Items for Systematic Reviews and Meta-Analyses (PRISMA; Liberati et al., 2009) and the guidelines of Enhancing Transparency in Reporting the Synthesis of Qualitative Research Statement (ENTREQ; Tong et al., 2012). The details of the process are provided in the following sections and Supplementary Information 1.

\section{Search Strategy}

English full-text studies published in academic journals in or after 2010 are included in this research. The search was conducted in databases including Web of Science, Scopus, PsycINFO (via Ovid), PubMed, Family and Society Studies Worldwide (FSSW, via EBSCO), and the databases sit within the ProQuest platform [including Education Resources Information Center (ERIC), Education Database, Psychology Database, Social Science Database, and Sociology Database]. Search strategies were refined for each database, and the details of the databases and search fields are provided in Supplementary Information 2. The following restrictions were applied equally in these databases: (1) The publication years were limited to "in or after 2010". (2) The publication language was limited to English. (3) Only articles published in academic journals were included. Books, book chapters, theses or dissertations, reports, working papers, conference papers and proceedings, and other grey literature were excluded.
The search terms are a combination of keywords related to "children", "migration", "agency" and "family". The search terms were:

(1) child* OR kid* OR adolescent* OR youth* OR teen* OR student* OR minor OR junior

(2) migrant* OR migrat* OR immigrant* OR immigrat* OR floating OR left-behind

(3) agency OR decision-making OR self-determination OR autonomy OR voice

(4) family OR household OR domestic OR home OR parent* OR father* OR mother* OR paternal OR maternal OR caregiver* OR carer*

The main search was conducted in April 2020. Articles were first screened by title and abstract, and then closer examinations were conducted after the screening. The original number of search records was 2935 . Of these, 42 were excluded because they were published in books or book sections rather than academic journals. After removing the duplicates, 1580 records were included in the title and abstract screening, and 1485 records were excluded with reasons. In the title and abstract screening, when the first researcher was not sure whether the articles should be included, the research team discussed the articles to achieve consensus. This resulted in 95 articles being included for the full-text screening. Of these, 39 articles were excluded with reasons, and 56 articles were considered to be included in the quality assessment. In the full-text screening, after the first researcher completed the full-text screening, the second researcher checked the included and excluded articles. Consensus on the included and excluded articles was achieved. The 56 identified articles were assessed for eligibility in the full article.

A backward tracking strategy was conducted to identify additional relevant articles. The reference lists of the 56 identified articles were examined for additional articles published in or after 2010. Another nine relevant articles were included by checking the title and abstract of studies in the reference lists. In summary, 65 articles were included to do the quality assessment. A flow chart of the systematic literature search and screening is developed according to PRISMA guidelines (Liberati et al., 2009) (see Fig. 1).

\section{Inclusion and Exclusion Criteria}

The detailed inclusion and exclusion criteria are:

(1) Participants are children who are younger than 18 years old. The children include those influenced by migration, such as migrant children, immigrant children, leftbehind children, children of immigrants, and secondgeneration immigrants. Studies of child marriage, early 
Fig. 1 A flow chart of the systematic literature search and screening. Note the detailed reasons for excluding articles in the title and abstract screening are provided in Supplementary Information 3

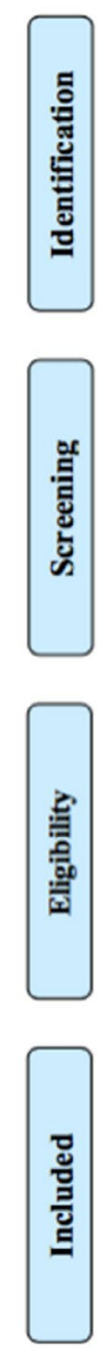

marriage(s), teenage pregnancies, and teenage mothers and related topics, including their pregnancy, giving birth, childbearing, contraception knowledge, and attitudes, were excluded, though they belonged to the children's age group. Studies focusing on the migration of young football players, which is closely related to the context of the football industry, were excluded. Studies only focusing on university students, college students, emerging adults, and young adults were excluded considering that most of them were older than 18 years old. Studies that included young people both younger and/ or older than 18 were included.

(2) The articles should be focused on the behavioral aspect of agency. Studies only examining children's percep- tions of agency or sense of agency which did not involve exercising agency were not included.

(3) Empirical research articles, using either quantitative, qualitative or mixed methods were included. Comments, literature reviews, systematic reviews, metaanalyses, and theory papers were excluded.

(4) The article examined children's agency in the family setting. Children exercising agency outside the family, such as agency in classrooms, schools, hospitals, organizations, and the community, were excluded.

(5) The included studies had to be focused on migrant families, including domestic migration and international migration. Forced migration such as refugees and asylum seekers were not included in this research as the 
migration decision may be out of control of the parents. However, studies that compared migrants/immigrants, refugees, and asylum seekers were included, and the review focuses on the findings of ordinary migrants.

In addition, studies that included insufficient data for analysis were excluded. Articles that did not have a fulltext version were excluded. However, no studies met these exclusion criteria in the current review. Studies published in non-academic journals or unpublished studies (e.g., Ph.D. or master's Dissertations) were excluded.

\section{Data Extraction}

Data extracted with the selected studies included the author(s), year, country, publication journal, type of study, characteristics of participants (including the type of migration), methods, measures of agency (what is considered as exercising of agency in qualitative studies), and findings of agency. The extracted information is shown in Table 4 in the "Appendix". Measures and findings of agency are summarized in the "Results" section. Two researchers were involved in the coding of each article included. The first researcher extracted the data, and the second researcher checked the extracted data.

\section{Quality Assessment}

Two researchers independently assessed the quality of all identified articles based on quality checklists (the "QualSyst" tool), developed by Kmet, Cook and Lee (2004). This tool consists of two checklists: one is designed for quantitative studies, and the other for qualitative studies. The selected quantitative and qualitative studies were assessed based on the two checklists respectively, while the mixedmethods studies were assessed based on a combination of the two checklists. The two checklists have clear scoring systems and were used in the previous systematic review of qualitative, quantitative, and mix-methods studies (Reardon et al., 2017). The modified items of the two checklists are provided in Supplementary Information 4.

Articles were rated on the items in both checklists using a three-point scale $(2=$ Yes, $1=$ Partial, and $0=$ No). The quality score is the percentage of total score among the possible maximum score. Based on the calculated quality scores, the articles were categorized as strong quality ( $\geq .75$ ), moderate quality (between .55 and .75), and weak quality $(\leq .55)$ (Landais et al., 2020). When there was disagreement on the quality category, the consensus was reached through discussion. Cohen's $\kappa$ coefficient evaluated the interrater reliability. The interrater reliability between the two researchers was achieved with a high level of agreement $(\mathrm{k}=.845)$. The inclusion or exclusion of the assessed articles was determined by the mean scores of the two independent researchers. Based on the rating, articles with moderate to strong quality were included. All the articles have strong or moderate quality, and thus no article was excluded.

\section{Data Analysis}

Framework synthesis (Gale et al., 2013) was conducted with the included studies. The stages include: familiarization with the included studies, developing a working analytical framework, applying the analytical framework, charting data into the framework, mapping, and interpreting (Gale et al., 2013; Lee, Lo, \& Ho, 2018). The analytical framework was developed based on the review questions and the stages of migration, including children's agency in the migration decision-making process, children's agency in daily life during/after migration in different types of migration, and how migration shapes household dynamics. Practically, first, the keywords and sentences in each article were identified, then the common patterns and themes across different articles were identified, and finally, these key findings were compared and synthesized. The analyzing procedures are similar to those in qualitative studies ( $\mathrm{Li}, 2010)$.

\section{Results}

\section{Characteristics of the Included Articles}

In total, 65 articles published between 2010 and 2020 were included in the systematic review (see Table 4 in the "Appendix"). Out of the 65 selected articles, 41 are qualitative studies, 16 are quantitative studies, and 8 are mixedmethods studies (see Table 1). In the current review, studies that used only qualitative data in a mixed-methods project were counted as qualitative studies (e.g., Hoang \& Yeoh, 2015). Studies using a quantitative approach to analyze qualitative data were treated as qualitative studies (e.g., Bakken \& Brown, 2010). One quantitative article study only used qualitative quotations in the discussion section and thus was considered a quantitative study rather than a mixed-methods study (e.g., Jensen \& Dost-Gözkan, 2015). In total, the 65 included studies reported five types of children's involvement in migration: (1) being left behind (9 studies), (2) (im-)migrating with parents (43 studies), (3) migrating independently (6 studies), (4) family reunification (parents migrate first, and then children migrate to join the family, that is children have both experiences of being left behind and migrating, 4 studies), ${ }^{2}$ and (5) return migration

\footnotetext{
2 Articles that mentioned family reunification but did not focus on it were not labelled as such.
} 
Table 1 Characteristics of the included articles

\begin{tabular}{lllll}
\hline Children's migration status & \multicolumn{2}{l}{ Type of study } & Total \\
\cline { 2 - 4 } & $\begin{array}{l}\text { Mixed-meth- } \\
\text { ods }\end{array}$ & Qualitative & Quantitative & \\
\hline (Im)migrant children with parents & 1 & 27 & 15 & 43 \\
Left-behind children & 4 & 5 & 0 & 9 \\
Independent child (im-)migrants & 2 & 3 & 1 & 6 \\
Family reunification & 0 & 4 & 0 & 4 \\
Return (im-)migrant children & 1 & 2 & 0 & 3 \\
Total & 8 & 41 & 16 & 65 \\
\hline
\end{tabular}

Table 2 Children's levels of agency in the migration decision-making process

\begin{tabular}{ll}
\hline Levels of agency & Types and locations of children \\
\hline High-level agency (make own decisions) & Independent child migrants in Ghana (Amoah, 2020; Kwankye, 2012; Mizen \& Ofosu-Kusi, 2013) \\
& Unaccompanied children in Sweden (Lalander \& Herz, 2018) \\
& Unaccompanied minors from Zimbabwe to South Africa (Adefehinti \& Arts, 2019) \\
& Some returned migrant children to Mexico (Zúñiga \& Hamann, 2020) \\
& Returned migrant children from U.S. to Mexico (González et al., 2016) \\
& Some immigrant children from English-speaking backgrounds in Australia (Hutchins, 2011) \\
Middle-level of agency & Becoming a young migrant or a young stayer in Lao (Huijsmans, 2014) \\
& Children in intra-EU migrant worker families (Moskal \& Tyrrell, 2016) \\
Low-level of agency or agency within a & Children migrating from China to Singapore with study mothers (Huang \& Yeoh, 2011) \\
restricted framework of choices & Young Turkish migrants in U.K. (Tanyas, 2012) \\
& Left-behind children in Vietnam (Hoang \& Yeoh, 2015) and Romania (Pantea, 2011) \\
& Returned migrant children to Mexico (Zúñiga \& Hamann, 2020) \\
& Some immigrant children from English-speaking backgrounds in Australia (Hutchins, 2011) \\
& Overseas born youth returned to Tonga (Lee, 2016) \\
& Some independent child migrants (Kwankye, 2012)
\end{tabular}

(children who return to their parents' birthplace with parents or without parents, 3 studies). The largest proportion of studies were conducted in the U.S. (24 studies). The samples in the 65 studies include preschool children, primary schoolaged children, adolescents, and young adults, while most of them were aged 5-18. ${ }^{3}$ These articles have mainly examined children's agency in the migration decision-making process and children's agency in daily life during/after migration. In the following sections of the analyses, the descriptive statistics of each article, such as the number of cases studied was not discussed individually, and it is shown in Table 4 in the "Appendix".

\section{Quality Assessment of the Included Articles}

The detailed quality assessment is provided in Tables 5, 6, and 7 in the "Appendix". Specifically, 17 studies have moderate quality, and 48 studies have strong quality. Considering the types of studies, 6 mixed-methods studies (75\%) have moderate quality while only $2(25 \%)$ have strong quality,

$\overline{3}$ Some of the studies include young people older than 18.
11 qualitative studies (27\%) have moderate quality and 30 qualitative studies (73\%) have strong quality, and all of the 16 quantitative studies (100\%) have strong quality.

\section{Children's Agency in Migration Decision-Making}

Decisions related to migration include: "parents migrating and children staying behind", "parents migrating together with children", "children migrating independently", "family reunification", and "parents' or children's returning and re-emigration". The levels of children's agency are reflected by the level of children's participation in making migration decisions. Children who can make decisions independently have a high level of agency, while children who do not get involved in migration decisions have a low level of agency. Some children can have certain influence on the decisions related to migration, between low-level and highlevel agency. In the 65 articles, all three levels of agency could be observed. These three levels of exercising agency in the migration decision-making process are summarized and compared in Table 2. It should be noted that one article may report children's varied levels of agency in migration decision-making. 
First, some articles described circumstances in which children had high levels of agency in their migration decision-making process, especially among independent child migrants. For example, some children made individual choices to leave their households and become independent migrants in Ghana, which was related to their need to express their agency (Amoah, 2020; Mizen \& OfosuKusi, 2013). However, significant gender differences exist among independent child migrants. Among the 451 independent child migrants in Ghana, two-thirds of the 293 female migrants stated that they made their own decisions to migrate, while more than half of the 158 male migrants stated that their migration decisions were made by persons other than themselves (Kwankye, 2012). Some unaccompanied children in Sweden (Lalander \& Herz, 2018) and unaccompanied minors from Zimbabwe to South Africa (Adefehinti \& Arts, 2019) also decided to migrate independently. Although most children were not involved in the decision to return to Mexico, some saw themselves agentively as actors to choose where they wanted to live and with whom they would live (Zúñiga \& Hamann, 2020).

Second, children had a moderate level of agency and could influence parents' migration decision-making in certain ways or at different stages of migration or remigration. For returned migrant children from the U.S. to Mexico, children played critical and varied roles in the family decisions on return migration and re-emigration. For example, parents consulted with the children in their decision to return or re-emigrate, and there were cases of children influencing parents on where to move, school choice, whether to return or re-emigrate (González, Cantú, \& Hernández-León, 2016). The process of family return migration provided an opportunity for children to renegotiate the parent-child relationships and exert their influence on decisions that directly affect their lives (González et al., 2016). For immigrant families from English-speaking backgrounds in Australia, in the majority of families, children actively attempted to influence adults, and some of them successfully influenced their parents on the migration decision, while the opinions of other children were overridden by the adults (Hutchins, 2011). In addition, one study conducted in Lao states that young people's agency was not limited to migration and may also be exercised through becoming or remaining a young stayer, and it was shaped by the intergenerational contract between parents and children (Huijsmans, 2014).

Third, children exercised little agency in the migration decision-making process, especially migrant children, leftbehind children, returned migrant children, and some independent child migrants. For example, in intra-EU migrant worker families, some children were encouraged to engage in family migration decision-making, but it was usually within a restricted framework of choices outlined by parents (Moskal \& Tyrrell, 2016). Children migrating from
China to Singapore with study mothers (Huang \& Yeoh, 2011) and young Turkish migrants in the U.K. reported their lack of active involvement in the family's decision-making processes (Tanyas, 2012). For left-behind children in Vietnam (Hoang \& Yeoh, 2015) and those in Romania (Pantea, 2011), their voices were largely not heard in the migration decision-making process and agreed that parents tended to underestimate their level of competence. The migration decisions of some independent child migrants were made by persons other than themselves (Kwankye, 2012). Overseas born youth returned to Tonga under pressure from their families, and they had been forced or their parents had decided to return (Lee, 2016). Although children in these studies had a low level of agency in making migration decisions, parents from different cultural backgrounds tended to say the decisions to migrate or return to homeland were made in the best interests of the children, to protect them or otherwise attend to their well-being, such as the parents of left-behind children in Romania (Pantea, 2011), parents of returned migrant children in Mexico (Zúñiga \& Hamann, 2020), and parents of some immigrant children in Australia (Hutchins, 2011).

\section{Children's Agency in Daily Life After Migration}

Children exercise their agency in everyday life within the family. Children's agency was expressed and exercised through various aspects in daily life, such as their socializing times, friendship groups, and negotiations and conflicts with parents (Clayton, 2013). Furthermore, children's agency is expressed in different ways and different aspects when facing different types of migration (see Table 3). There are many decisions and activities that children and parents need to consider in daily life during and after migration. Among the included studies, the decisions and activities related to children's agency in daily life after migration include a wide range of different activities.

\section{Migrant/Immigrant Families}

Migrant/immigrant children's agency is expressed in various ways. First, information disclosure and secretive behavior reflect immigrant children's autonomy (Bakken \& Brown, 2010). Adolescents controlled what to disclose to parents, and they had the autonomy to decide when they disclosed information and the type of information they wanted to disclose (Fernandez, Loukas, \& Pasch, 2018), and non-disclosure was used as an adaptive strategy to pursue autonomy in harmony with parental regulation (Yau, 2016). CanadianArabic immigrant adolescents also controlled what was told and presented to their parents when sharing information with parents (Ashbourne \& Baobaid, 2014). Similarly, Russianspeaking youth in Canada chose to hide their new identity 


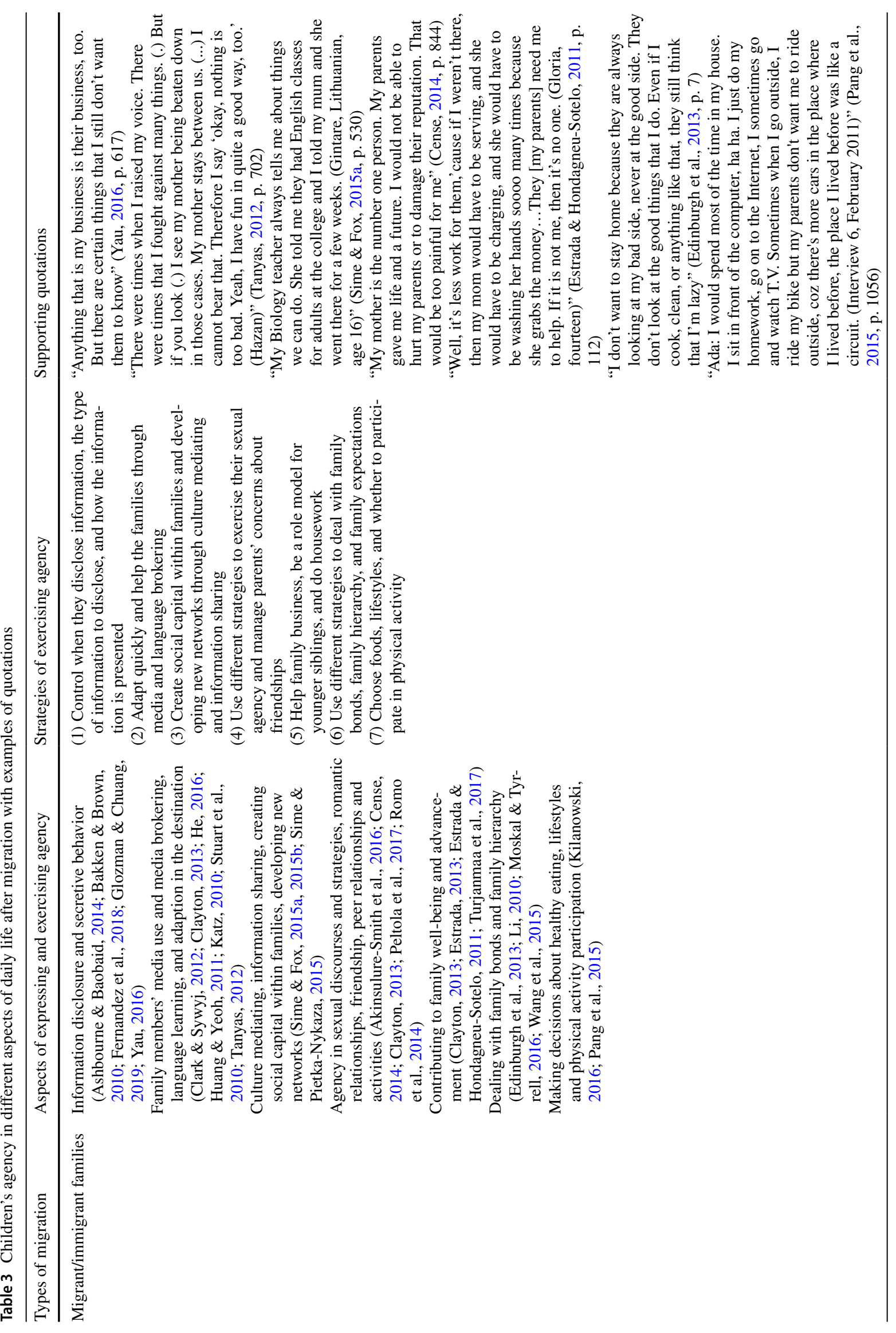




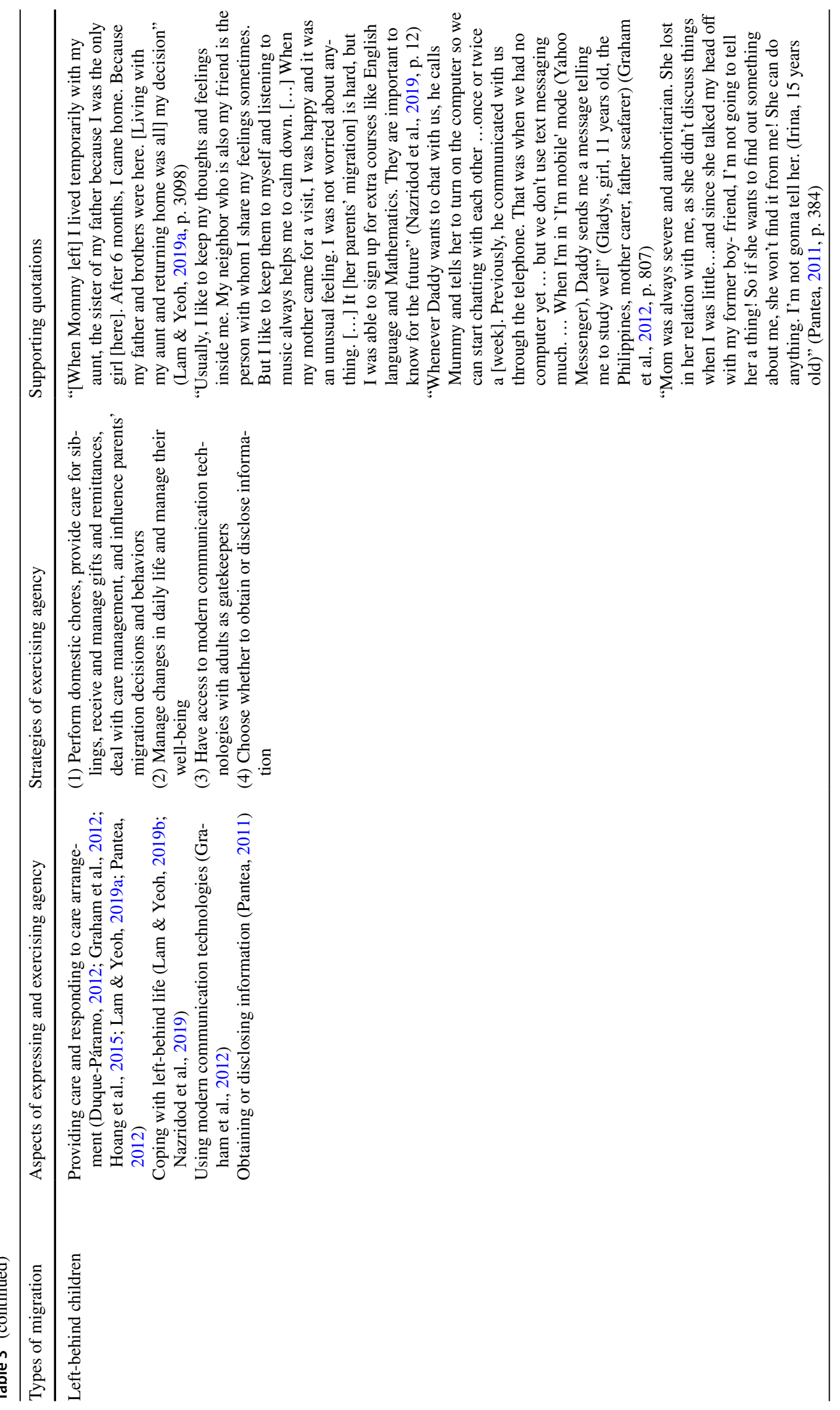




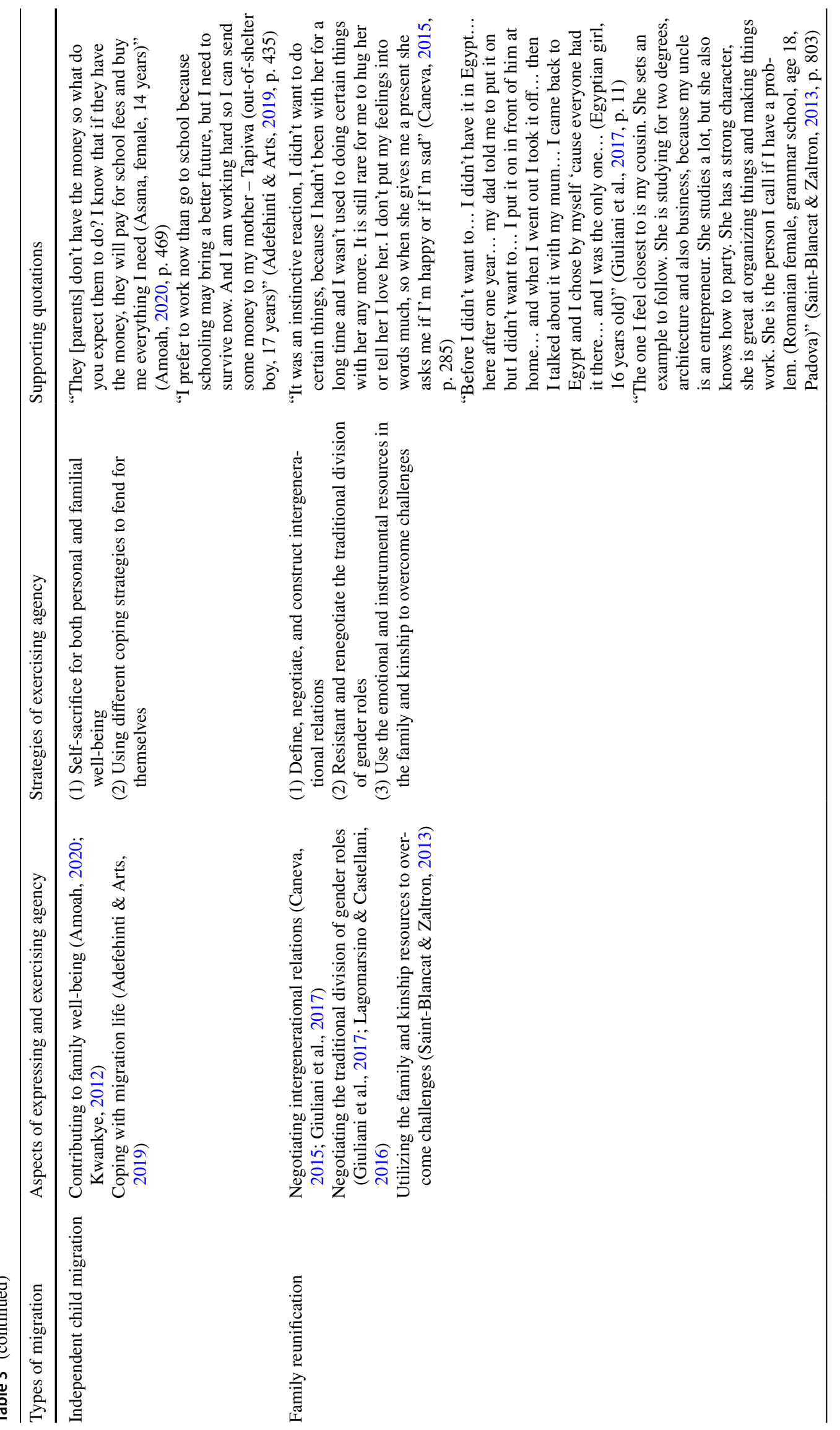




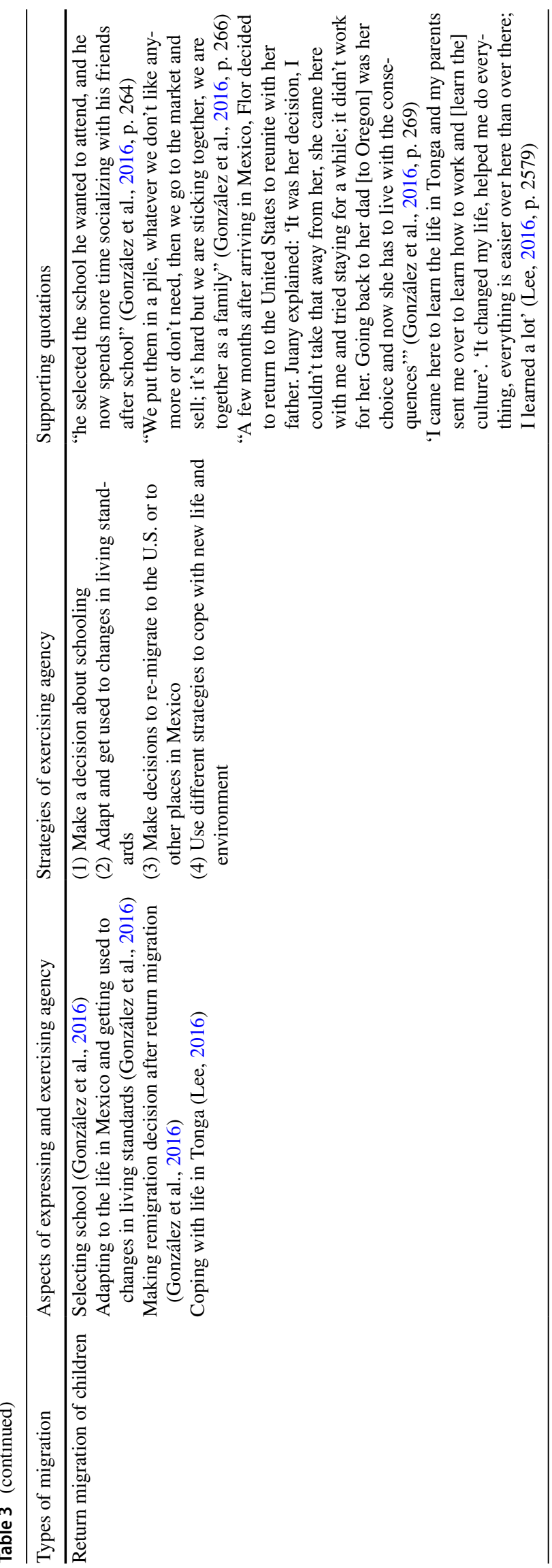

(particularly about religion) to avoid conflicts with their parents (Glozman \& Chuang, 2019).

Second, children's media brokering, language brokering, and adaption process reflect their agency. After family migration, children's agency tended to change as children adapted to new environment much more faster than their parents, and children had the opportunity to make more independent decisions, especially in international migration where parents did not speak the language of the hosting country (Huang \& Yeoh, 2011). Influenced by the school and peer groups, immigrant children adapted to the new culture and were more flexible with the norms and behaviors in New Zealand more quickly than their parents (Stuart et al., 2010). Similar findings are reported in the U.K., and the daughter-mother relationships were disrupted or transformed as mothers became more dependent on their daughters in the new country, while fathers survived and succeeded in challenging new conditions (Tanyas, 2012). As summarized by Tanyas (2012, p. 705), "the degree of adjustment required in the context of family migration is closely linked with shifting dynamics in familial and gender roles." Children have different actions; for example, British Chinese Children exercised agency in helping and teaching their parents about the new language or culture (Clayton, 2013). Immigrant teens in the U.S. helped their families to use the keyboard in their mother language, look up recipes, read news, and find movies from the native country (Clark \& Sywyj, 2012) and connected their parents to community institutions and organizations (Katz, 2010). The Chinese immigrant children played an important role in shaping their parents' discourse in the U.S. (He, 2016).

Third, migrant children exert agency in creating social capital within families and developing new networks through culture mediating and information sharing, such as the Eastern European migrant children (Sime \& Fox, 2015a, 2015b). For newly arrived Polish children in Scotland, facing the manifest tensions between the new culture and the Polish culture in their everyday lives, they had to respond in a continuous process of acceptance or resistance and exercise their agency in the processes of intergenerational learning and relations and family acculturation (Sime \& PietkaNykaza, 2015).

Fourth, children exercise agency in sexual discourses and strategies, romantic relationships, socializing opportunities, friendships, and peer relationships and activities. Children adopted different strategies to exercise their sexual agency, including "conforming to parents' values, breaking up with parents, leading a double life, and integrating competing discourses" (Cense, 2014, p. 835). Similarly, young people who migrated to Finland could choose whether to comply with their parents' views or questioning them and show a high level of agency in romantic relationships (Peltola et al., 2017). Some Latina girls had ever had at least one boyfriend, 
but only half of them reported that their mothers knew about it; these girls showed non-disclosure in boyfriend relationships (Romo, Mireles-Rios, \& Lopez-Tello, 2014). West African immigrant children were aware of their parents' concerns about their friendships and the efforts taken to prevent their friendships, and they adopted different strategies to deal with parents' concerns and efforts, including maintaining their friendships with both African and nonAfrican peers and adhering to their parents' requests and contending that friends served as a distraction from their education (Akinsulure-Smith et al., 2016). British Chinese children actively shaped and negotiated their socializing activities, such as resisting activities arranged by parents (Clayton, 2013).

Fifth, children exercise agency in the aspect of contributing to family well-being. Children's agency in the street vending activities shows they were not the "baggage" that adult immigrants simply brought along, but they were contributors to family processes (Estrada, 2013; Estrada \& Hondagneu-Sotelo, 2011). The Latino street vendor children contributed to their family's economic well-being and advancement. The children recognized the economic constraints in their families, felt an obligation toward their families, and showed gratitude for and reciprocate their parents' support. In addition to helping family business, children also did housework, were a role model for younger siblings, and provided child care for younger siblings to take on familial responsibilities (Clayton, 2013; Turjanmaa, Alitolppa-Niitamo, \& Jasinskaja-Lahti, 2017).

Sixth, children exercise agency in the aspects of dealing with family bonds and family hierarchy. Hmong immigrant adolescent girls in the U.S. decided to run away, return home, leave again, or stay home due to four main reasons, including "fighting restrictions", impulsive decision-making to go out to play, run away with minimizing the dangers, and returning home because of family bonds and wanting to be a good person (Edinburgh, Garcia, \& Saewyc, 2013). Asian American young people reported that they wanted their parents to recognize who they were and rebelled against the hierarchies, including family, when they were young (Wang, Koh, \& Song, 2015). For recent Chinese immigrant adolescents in Canada, influenced by immigrant poverty, unwavering parental support, and traditional Chinese culture, they either kept problems to themselves to avoid more family conflict, felt indebted to parental sacrifice, or shouldered family responsibilities at a tender age (Li, 2010). Migrant children from Central and Eastern Europe to Scotland or Ireland tended to function well despite periods of separation from parents and/or other family members, and they actively link the transnational families in social, emotional, and economic aspects (Moskal \& Tyrrell, 2016).

Seventh, children make decisions about healthy eating, lifestyles, and physical activity participation. For Latino immigrant children in the U.S., healthy decision-making includes fruits, vegetables, and physical activity. Mothers influenced health and healthy eating, while friends encouraged unhealthy food choices (Kilanowski, 2016). These children felt varying degrees of uncertainty about healthy foods in social situations and make poor-quality decisions. One study has examined Chinese migrant young people's agency in negotiating their lifestyles and physical activity participation in Australia. The findings show that young people, especially girls, lacked agency and critical discourse with their parents. The traditional Chinese family power relations limited their agency, and they often did not reach an agency of practice (Pang, Macdonald, \& Hay, 2015).

\section{Left-Behind Children}

Left-behind children's agency is expressed in the following ways. First, left-behind children were both care receivers and care providers in the context of migration. Young people's experiences of care provision in transnational families reflect children's agency (Pantea, 2012). For left-behind children in both Indonesian and Filipino, the absence of mothers may create vulnerabilities related to emotional support and lack of attention, and children were required to support mother's double burden by performing domestic chores (Graham et al., 2012). Colombian children whose parents have migrated exercised agency in receiving and managing gifts and remittances and providing care for siblings; however, scholars have conflicting opinions about sibling caretaking. In the negative aspect, it is a type of child labor and not appropriate to children's age and development; in the positive aspect, it is considered as acquiring autonomy and new responsibilities (Duque-Páramo, 2012). In addition to children's care provision, left-behind children in South-east Asia exercised their agency in responding to care arrangements, which comprised three sets of responses, including resilience, reworking, and resistance (such as refusing to engage with the adults' agendas) (Hoang et al., 2015). While many left-behind children in South-east Asia had no say over their care arrangements, some were able to assert their agency in influencing their parents' decisions and eventually migratory behaviors (Lam \& Yeoh, 2019a).

Second, children cope with their left-behind life. They manage changes in their life and manage their well-being. Left-behind children in South-east Asia had agency in 
determining migration outcomes, playing an active role in managing their own well-being, coping with parental absence, and keeping the family together. Their agency was situated within the confines of a migrant household in various unique ways (Lam \& Yeoh, 2019b). Left-behind children in Tajikistan showed resilience and can use coping strategies in their daily life to deal with the psychosocial costs of separation from migrant parents, and gender differences existed in their coping mechanisms. For example, boys spent their leisure time on social media networks and hung out or played sport with other boys, while girls felt overwhelmed by the caregivers' household chores (Nazridod et al., 2019).

Third, children exercise agency in initiating communication with migrant parents. Left-behind children in the Philippines had access to modern communication technologies for connection and contact and the opportunity to practice individual agency in different ways. However, adults often acted as gatekeepers in the process (Graham et al., 2012).

Fourth, children are active actors in obtaining or disclosing information. Left-behind children in Romania gained power because they can choose to disclose or withhold information, while migrant parents may lose power while losing information. Those in grandparents' care may try to undermine their control by manipulating their reduced mobility or computer illiteracy (Pantea, 2011).

\section{Independent Child Migration}

Independent child migrants were engaged in economic activities in Ghana, and they wanted to express their agency by contributing to family well-being and adopt strategies to reduce the burden faced by their parents or guardians (Amoah, 2020); many of them sent home remittances for household consumption (Kwankye, 2012). Unaccompanied minors from Zimbabwe to South Africa coped successfully despite being caught up in tensions between structure-agency and vulnerability-resilience; they neither expressed agency or resilience alone nor navigated vulnerability in isolation before, during, and after their migration journeys (Adefehinti \& Arts, 2019).

\section{Family Reunification}

After reunifying with families who are migrating, children can exercise their agency in different aspects in the migration destination. First, children exercised their agency in negotiating intergenerational relations after reuniting with their mothers in Italy (Caneva, 2015). Children can define the kin relations and mothers' roles and contribute to constructing the relations (Caneva, 2015). For Muslim immigrant adolescents reunified with their families in Italy, though obedience to parents was internalized in their values, they still mentioned some possible spaces for negotiation with the family (Giuliani, Olivari, \& Alfieri, 2017). Second, children can negotiate gender roles. For reunified teenage daughters of Ecuadorian migrants both in Ecuador as well as in Southern Europe, they participated in the migration project of the transnational family, but simultaneously they implemented practices of resistance and renegotiation addressing the traditional division of gender roles; as caretakers, they provided care in the transnational household, both in the origin and destination, while showing a higher commitment to school; meanwhile, they negotiated their role both inside and outside of the family, challenging parents' moral and sexual control strategies (Lagomarsino \& Castellani, 2016). Muslim immigrant girls also dealt with the choice to wear the veil, such as putting on the veil in front of the father at home and taking it off when going out (Giuliani et al., 2017). Third, they can utilize the family and kinship resources to overcome challenges. Young immigrant Romanians and Moroccans in north-eastern Italy can transform the family's material and emotional support into resources to overcome other challenges after re-joining their family (Saint-Blancat \& Zaltron, 2013).

\section{Return Migration of Children}

Returned migrant children from the U.S. exercised agency in selecting the school to attend, adapting to life in Mexico, and getting used to living standards; some children decided to emigrate to the U.S. after returning to Mexico (González et al., 2016). For children of Tongan migrants who returned to their parents' homeland, their perceptions of agency significantly shaped their responses to the cultural and physical environment, and their life in Tonga may influence their family dynamics (Lee, 2016).

In addition to the aspects reviewed and summarized above, some quantitative studies have used scales covering several smaller decisions (various matters) to measure children's autonomy, independence, decision-making authority, and agency, such as staying out at night, friends, taking classes, dating, watching television, doing homework, doing household chores, going to bed, going back home after school and spending time outside school, spending money, clothes, hair, and makeup (Roche et al., 2015; Tran \& Raffaelli, 2020; Varner \& Mandara, 2014); or parent-child conflict frequency and intensity and children's autonomy expectations for various everyday domains, such as chores, schoolwork, curfew, dating, family obligations, and going out (Bámaca-Colbert, Umaña-Taylor, \& Gayles, 2012, 2014; 
Eichelsheim et al., 2010; Estrada-Martínez et al., 2011; Fung et al., 2017; Juang, Syed, \& Cookston, 2012; Roche et al., 2019; Titzmann, Gniewosz, \& Michel, 2015). One quantitative study compared the independence in decision-making, representing whether adolescents were allowed to make their own decisions and rules about matters in daily life, between different generations of immigrants using a scale (Hamilton, 2010). Another quantitative study used scales of parental authority, expectations for behavioral autonomy, and parent-child conflict and found little support for discrepancies on autonomy predicting conflict and cohesion (Jensen \& Dost-Gözkan, 2015). Youth's romantic autonomy in different activities was measured using a questionnaire (Dhariwal \& Connolly, 2013). Language brokering scale was employed to measure adolescents' situations of school language brokering, community language brokering, and home management language brokering (Roche et al., 2015). These smaller decisions can show children's agency in micro levels and can be compared with children's agency in major decisions to have a comprehensive understanding of children's agency.

\section{Migration Influencing Children's Agency}

Children's agency and degrees of freedom are highly variable and shaped by varied intersecting factors (Graham et al., 2012). Family members tend to have different interests, and family migration decision-making is based upon negotiating individual influence and power within the family, often at different stages in the process (Hutchins, 2011). Migration may lead to the change of household dynamics. In summary, children's agency development mainly has two aspects in the context of migration (Stuart et al., 2010; Turjanmaa et al., 2017): (1) Children's normative development issues. Children, regardless of whether they are left behind, migrating, or living with their parents in their hometown, may have issues about agency development during their normative developmental processes. (2) Migration's influence and the associated issues. Migration may have a positive or negative influence on children's experiences of agency. Migration and the associated issues may complicate the normative developmental processes (Stuart et al., 2010; Titzmann et al., 2015).

For children's normative development, children's agency is influenced by different factors, such as age, cohort, and ethnic group. Children are expected to exert more agency to make decisions independently when getting older. The abilities and levels of agency, granted by parents and acted on by children, increased with children growing older among British Chinese children (Clayton, 2013). Older African American adolescents had less full disclosure and more partial disclosure about activities and relationships with peers than their younger peers (Bakken \& Brown, 2010). Similarly, late adolescents had more nondisclosure issues than midadolescents among Chinese American families (Yau, 2016). Different generations of immigrants also have different levels of independence in decision-making; first-generation immigrants tended to have less independence in decisionmaking than their more recent counterparts (Hamilton, 2010). In addition, there are differences in levels of agency across different ethnic groups. For example, Hmong adolescents reported more frequent partial disclosure and topic avoidance in the younger age group (though not statistically significant) and provided significantly more relational justifications than their African American counterparts (Bakken $\&$ Brown, 2010). Different cultural groups may have different cultural meanings of autonomy and authority (Jensen \& Dost-Gözkan, 2015).

Migration could exert its influence on children's agency in positive or negative ways. On the one hand, migration disables people's capability, as migrants are situated in vulnerabilities compared with the local people; on the other hand, migration enables people's capability and enhances their autonomy (Punch, 2007; Sarausad, 2006). Possible explanations for migration enabling autonomy include: (1) Young people in the context of migration could contribute to their family well-being after migration, either contributing financially (Estrada \& Hondagneu-Sotelo, 2011; Punch, 2007) or providing care (Pantea, 2012; Turjanmaa et al., 2017), which is related to the family's material circumstances and cultural backgrounds. (2) Children's migration and transnational journeys are also journeys of personal development, shifting from dependence and passivity to actively figuring out specific strategies to cope with new environments; children state that they have changed a lot of bad habits and get to be mature faster after moving to Singapore from China (Huang \& Yeoh, 2011). Although children may have low levels of agency in the initial migration decision-making process, they have an increased level of agency during the migration process (Huang \& Yeoh, 2011; Tanyas, 2012). Migrants gradually adapt to new environments and learn how to exercise their agency in their lives (Punch, 2007). (3) Children's negative experiences in the context of migration give them some bargaining power in their families, partly because children become more mature, also because parents tend to be more lenient with their children due to feelings of guilt (Dreby, 2010; González et al., 2016). Nevertheless, parents may also want to provide more protection for their children in a new environment and control and monitor more in children's lives as parents are losing their ability to shape children's well-being and future (Akinsulure-Smith et al., 2016). (4) The cultural and social context in which family lives are lived, experienced, and negotiated also influence children's agency and parenting approaches after the 
migration (Clayton, 2013; Goh \& Kuczynski, 2009), especially fostering independence (Glozman \& Chuang, 2019) and challenging the gender-based norms (different in the sending origins and the destinations) (Giuliani et al., 2017). Household dynamics are changing in the process of migration, and different levels of authority and freedom occur in different stages of the migration cycle (Pantea, 2011). For example, migrant parents may lose control over daily actions, while previously subordinate groups, such as children, remaining parents, and grandmothers, gain authority positions; after reunifying with families, migrants may experience a sense of lost control and exclusion and revitalize the direct control and conflict of influence (Pantea, 2011).

In the context of migration, children's exercising of agency is also influenced by the intergenerational discrepancy and the conflicts of interests between them and their parents (Pantea, 2011) and the intergenerational ambivalence (Luescher \& Pillemer, 1998; Sime \& Pietka-Nykaza, 2015). On the one hand, the discrepancy could be interpreted as a developmental phenomenon, and adolescents need to strive for autonomy, whereas parents are concerned with maintaining order in the family and protecting their children from harm (Jensen \& Dost-Gözkan, 2015). On the other hand, left-behind children and parents have to deal with parent-child separation, and migrant parents and children are confronted with the acculturation gap, making them have different opinions on agency and autonomy and making children's normative development more complicated. The discordance in acculturation between parents and children may exacerbate the original problems and lead to new problems, or in the positive aspect, help to solve these problems. The intergenerational discrepancy, intergenerational conflict, or adolescent non-disclosure is not always considered as a negative component of parent-adolescent relationship, but is generally seen as a normal manifestation of the process of individual identity development and helpful to draw privacy boundaries (Kapadia \& Miller, 2005; Stuart et al., 2010; Yau, 2016).

The feminization of migration greatly influences children's agency. Mothers' migration usually complicates the household dynamics as it challenges traditional genderbased norms, and left-behind husbands and children have to hold new roles and responsibilities (Graham et al., 2012). Moreover, children whose parents get divorced during the migration process have to face different layers of family transformations (Pantea, 2011).

\section{Discussion}

Although an increasing number of studies have examined children's agency in the context of migration, there is not a systematic review to compare and summarize children's actions of exercising agency. This systematic review describes the current knowledge of children's and adolescents' behavioral aspects of agency within families in the context of migration. The review searched and assessed English-written articles published in academic journals in or after 2010, and 65 articles were included in the final review, including 41 qualitative studies, 16 quantitative studies, and 8 mixed-methods studies. These studies show children have different levels of behavioral agency in the migration-decision-making process, and children have different strategies to deal with daily living with independent migration, family migration, family separation, or return migration.

Children are not passive bystanders in the process of migration, and they socially navigate their lives, negotiate resources, express their feelings, and make decisions about their life (Abrego, 2014; Dreby, 2010; González et al., 2016; Huang \& Yeoh, 2011). As summarized by one study, "autonomy in decision-making was a continuous variable that reflected a continuum from parents making decisions independently to adolescents making decisions independently" (Varner \& Mandara, 2014, p. 676). Similarly, children's agency in the migration decision-making process and daily life after the migration is also a continuous variable ranging from a low level of agency (parents make decisions independently) to a high level of agency (children make decisions independently). Children and adolescents are not a homogeneous group; they have different migration and left-behind statuses, play different roles in the migration, and have varying levels of agency within families in the context of migration. In addition to the levels of agency of children across different contexts, children may also have varying levels of agency at different stages of migration in the same context (González et al., 2016; Huang \& Yeoh, 2011). In the initial decisions of migration or return migration, most children tend to have limited agency; however, they used different strategies to influence their parents and manage their well-being in daily life after the initial decision. For example, some children had low levels of agency at the initial migration decision-making stage; however, when migrant children adapted well and quickly to their destinations, they exercised high levels of agency to decide matters in their daily life (Huang \& Yeoh, 2011). Similar findings are also reported among left-behind children and returned migrant children. For example, some left-behind children exerted little agency about their parental migration decisions, but they exercised higher levels of agency as they could choose to disclose or withhold information to their migrant parents (Pantea, 2011). These children can also contribute to family well-being and deal with family bonds, hierarchy, and expectations. These findings show that children are not merely victims, passively influenced by migration. However, they have a certain level of personal growth and have different 
strategies to exercise their agency in different aspects of life living with migration, and the parent-child dynamics changes with their increasing personal growth.

Nevertheless, these findings do not mean that migration only positively influences children's agency and well-being. Children's vulnerability is manifest in previous research. Children's agency in the context of migration is not exclusively influenced by migration. Many factors play roles in children's exercising agency, and migration may complicate the situation (Pantea, 2012). Agency is not the same as wellbeing-children can have agency which undermines their wellbeing, for example they take risks, and for left-behind children they tend to have a lot of agency because they have little supervision, but on the other hand they are unhappy because their parents are away; immigrant children may also take care of various matters independently as their parents are busy with their work in the migration destination. Children may have ambivalent feelings about their agency and well-being in the context of migration.

This review has several methodological implications which can inspire future research design. First, agency is a context-based concept, behaviors in one context are considered exercising agency, while in another context, they are not considered exercising agency. For example, children providing care for other family members can be considered as exercising agency to respond to life challenges or as being victims who have to take on extra responsibilities (DuquePáramo, 2012). Such varied perceptions can be related to cultural difference. There is not a well-designed framework or questionnaire to make meaningful comparison of children's agency in different cultural backgrounds. Some scholars think it is problematic to quantify human agency and state that agency should be described qualitatively and contextually (Abebe, 2019; Durham, 2008).

Nevertheless, one publication argues that confining research to a "local" context may obscure important largerscale structures and phenomena, and thus agency could be situated within broader contexts (Hoang \& Yeoh, 2015). Although we agree that agency should be described contextually, it is still important to quantitatively measure children's agency. As scholars have mentioned, "the degree of agency, the impact of that agency, let alone the nature of that agency" is not clear (Bluebond-Langner \& Korbin, 2007, p. 242). What is the appropriate level of children's agency at different ages? What kind of agency should be considered to be good? These two questions still need further research, and one possible way to measure the appropriate level and type of agency is to measure whether the agency is suitable for children's short-term and long-term well-being. As it is challenging to know the appropriate level of children's agency in the short term, longitudinal study and follow-up study is needed to evaluate the long-term impact of children's agency and to evaluate what is the appropriate level and type of children's agency (Pang et al., 2015). Mixed-methods research is helpful to describe agency quantitatively, qualitatively, and contextually.

Second, both children's agency and vulnerability need to be considered in future research. Young people neither cope well with parental absence because they have agency nor are they unavoidable victims of their situations. The former supports the "policy approach that favors migration and underrates its costs for young people (politics of agency)," while the latter supports that a policy approach "that singles out young people with migrant parents as victims of migration (politics of suffering)" (Pantea, 2012, p. 252). Focusing exclusively on children's agency or children's vulnerability is both biased (Pantea, 2012). Children's agency is embedded within, expressed through, and influenced by societal provisions, processes, and institutions (Adefehinti \& Arts, 2019). Children could exert certain levels of agency to deal with their daily lives while they are influenced by various social, structural, economic, and environmental constraints (Choi, Yeoh, \& Lam, 2019). However, with limited resources in the constrained circumstances, some children are sensitive to these factors and more likely to be influenced and have a low degree of control over their life. It is important to help them remove the barriers and ensure equal access to resources, opportunities, services, and welfare.

Third, focusing upon children's agency does not mean to marginalize the important place of adults in children's lives, and it is important to pay attention to practices and processes that adults and children use to position themselves in relation to each other as children and as adults (Alanen, 2001; Mannion, 2007). Both children's and parents' perspectives (parent-child dyads) need to be included. Migrant children and young people have recognized their dependency on their families and the decisions made by their parents, either economically, role modeling, or in other ways; and children's formal and legal dependency on parents would end with their age approaching 18 (Rübner Jørgensen, 2016). Children's age is still an important factor when examining the parental influence on children's agency.

Fourth, scholars argue that agency is not only about the capability to achieve positive changes but also by the capability to endure, suffer, and persist when facing power and sociality, as indicated by research exploring the concept of "bonded agency" (Cense, 2014; Mahmood, 2001). Challenging the prevalence of freedom-centered approach in research of agency, the "alternative modes of agency" can be interpreted as "enduring and negotiating one's embeddedness 
in relations of power and thick webs of sociality" (Phạm, 2013, p. 29).

Fifth, children's agency does not need to manifest in "big" ways or lead to "major" changes (James \& James, 2004; Lam \& Yeoh, 2019b). The outcomes of exercising agency may not be immediate but may become evident in the long term. Exercising of agency is not a one-off event, instead it is an ongoing process. Children may have varying levels of agency in different aspects of their lives. Agency means simply decision-making and the broader capacity to intentionally act upon the world (Bluebond-Langner \& Korbin, 2007). Sixth, coupled with migration, the context of the COVID-19 pandemic may make children's agency more complicated. The new context should be considered in future research on children's agency.

In addition to the contributions, this review has some limitations. First, only English articles published in academic journals in or after 2010 were included, which may have bias, while other types of articles, articles published in non-English articles, and articles published before 2010 were excluded. Second, this review focused on the behavioral aspect of agency, while the perceptions of agency were not fully described. Third, this article focuses on children's agency within the family. In contrast, children's agency in the school, in the community, and in the social activities were not included, and children's agency outside their household may be related to their agency within the household. These aspects could be considered in future systematic reviews of children's agency. Fourth, some of the studies include young people younger and older than 18 years old, and some of the studies include both migrant children and native children or refugee children, which makes it difficult to count the number of children involved in or influenced by migration and aged $0-18$.

\section{Conclusion}

Decisions made by families have important implications for children, and the power dynamics within families may change when family members migrate. To better understand the state of the research, this systematic review summarizes the literature on children's exercising of agency in the family's migration decision and on a daily basis in the process of migration. The review revealed that children can have limited, moderate, and higher levels of agency in the migration related decisions. In different types of migration, children's agency is exercised in different aspects of daily life living with migration. Different activities can be considered as exercising of agency. Examples include children negotiating intergenerational relations after family reunification, left-behind children's information disclosure and secretive behavior, migrant children's media, and language brokering, and so on. Various factors play a role in children's agency and normative development, and migration can influence children's agency either positively or negatively and may make children's normative issues more complicated. Children's agency is important to their well-being, and it is exercised in specific spaces and places. Adults who work with children should pay attention to children's voices and perspectives and understand children's exercising of agency in specific spaces and places.

\section{Appendix}

See Tables 4, 5, 6 and 7. 


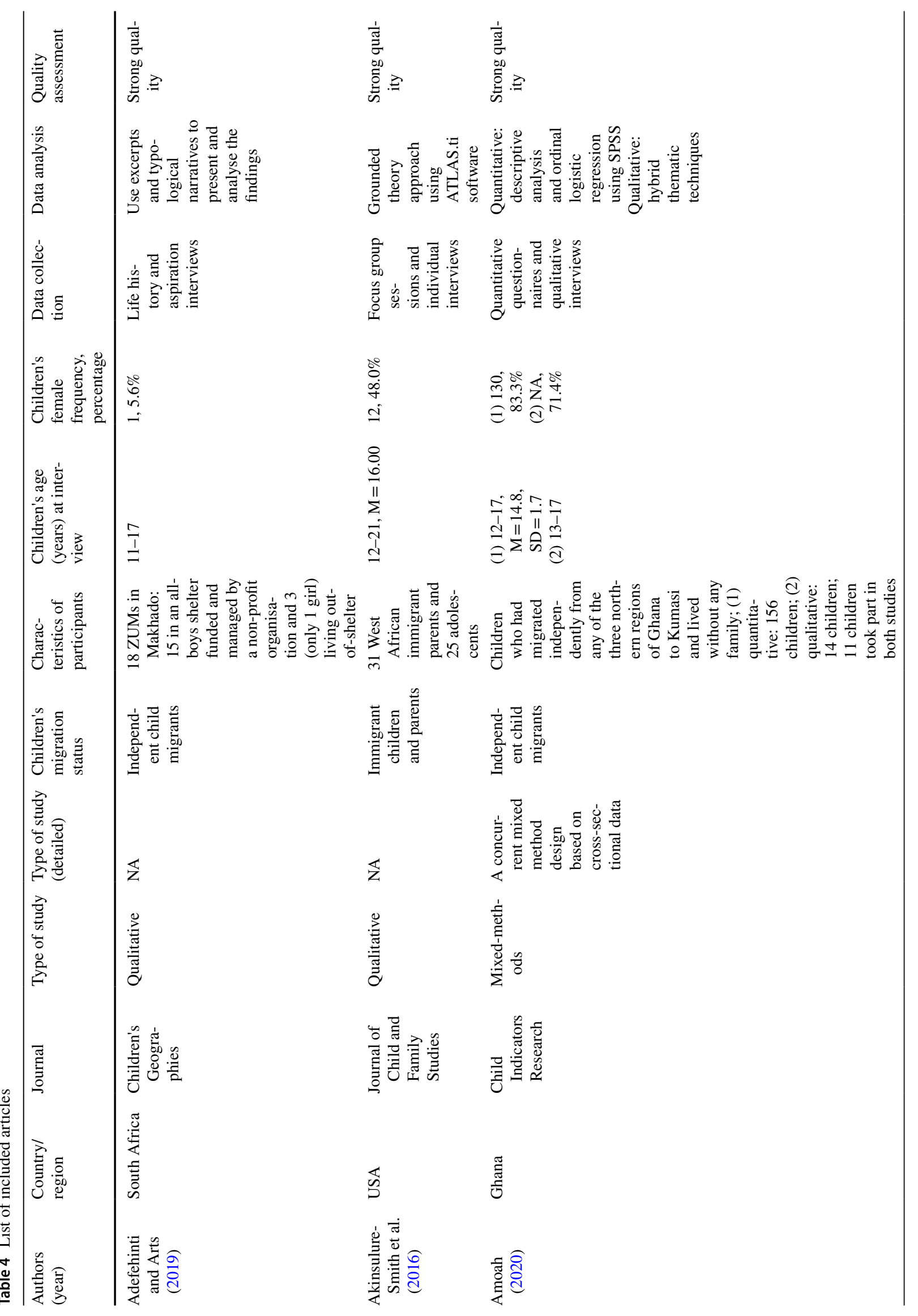




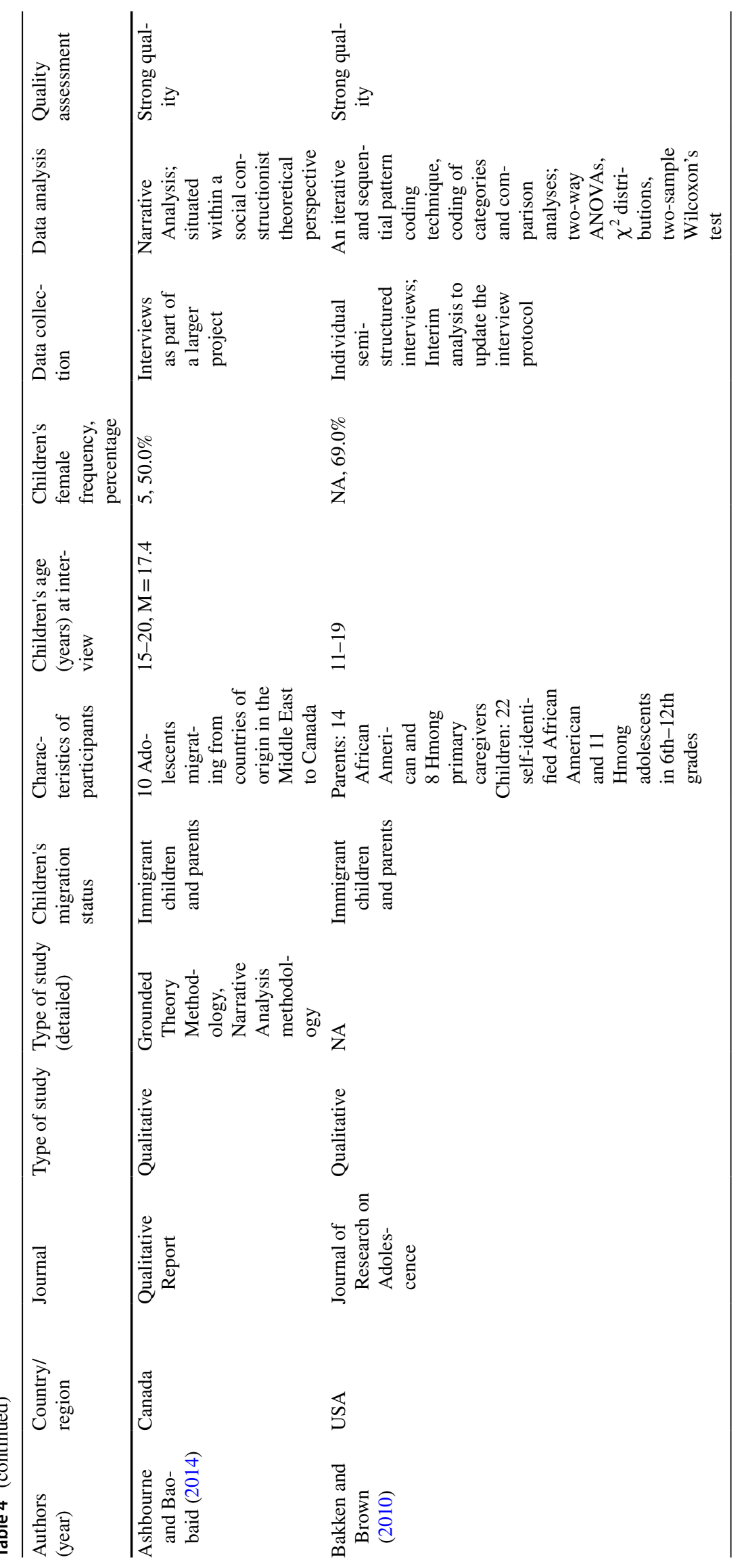




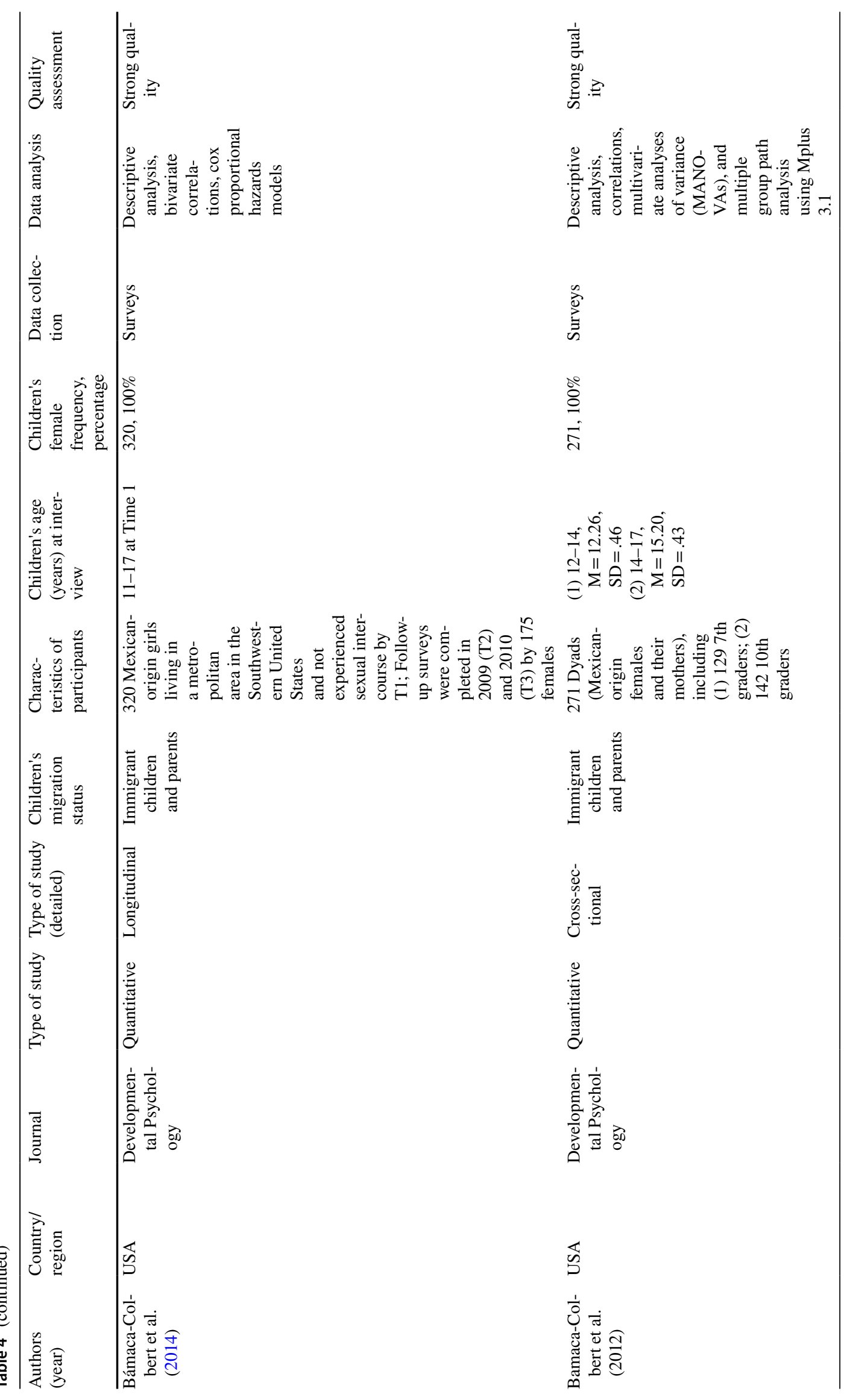




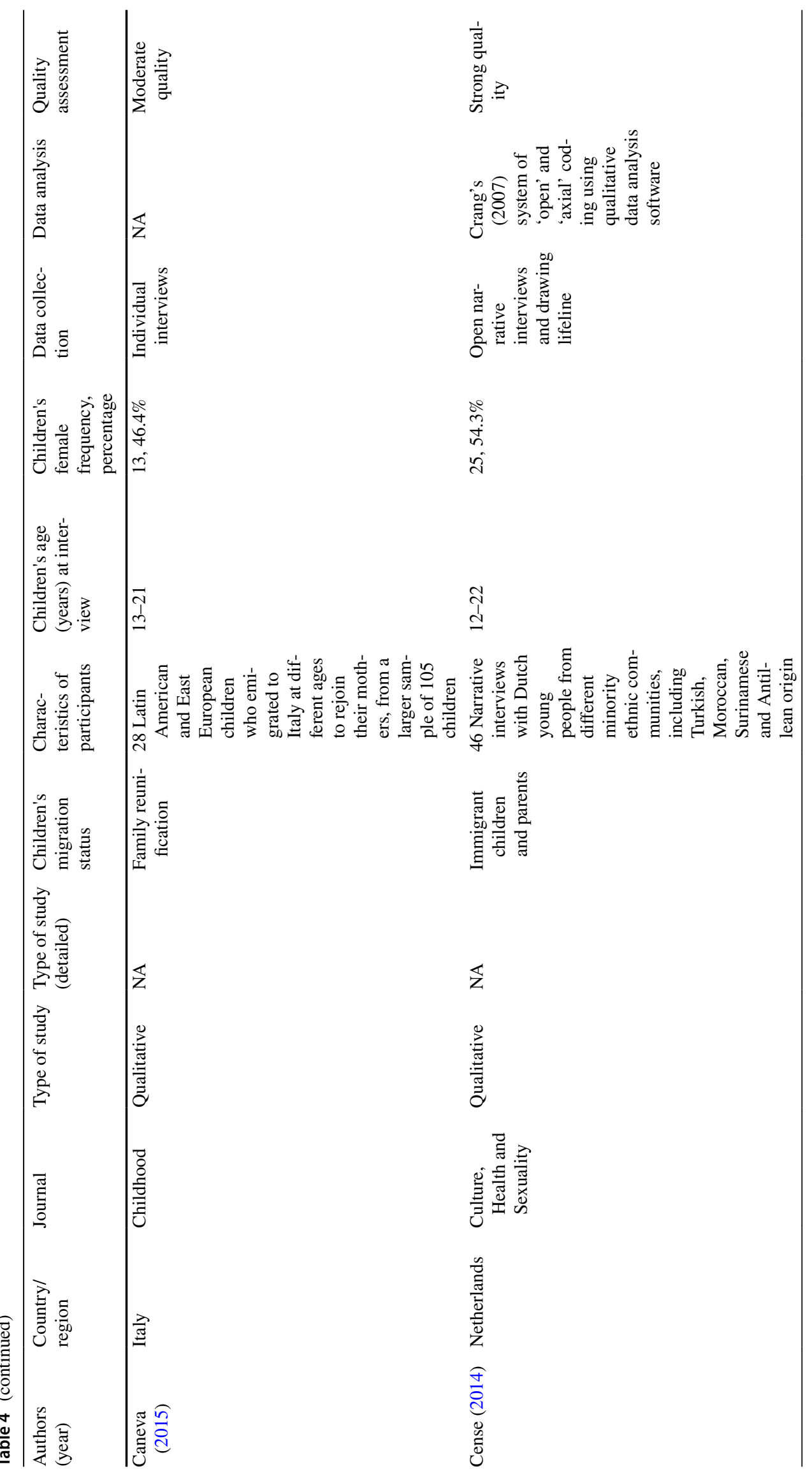




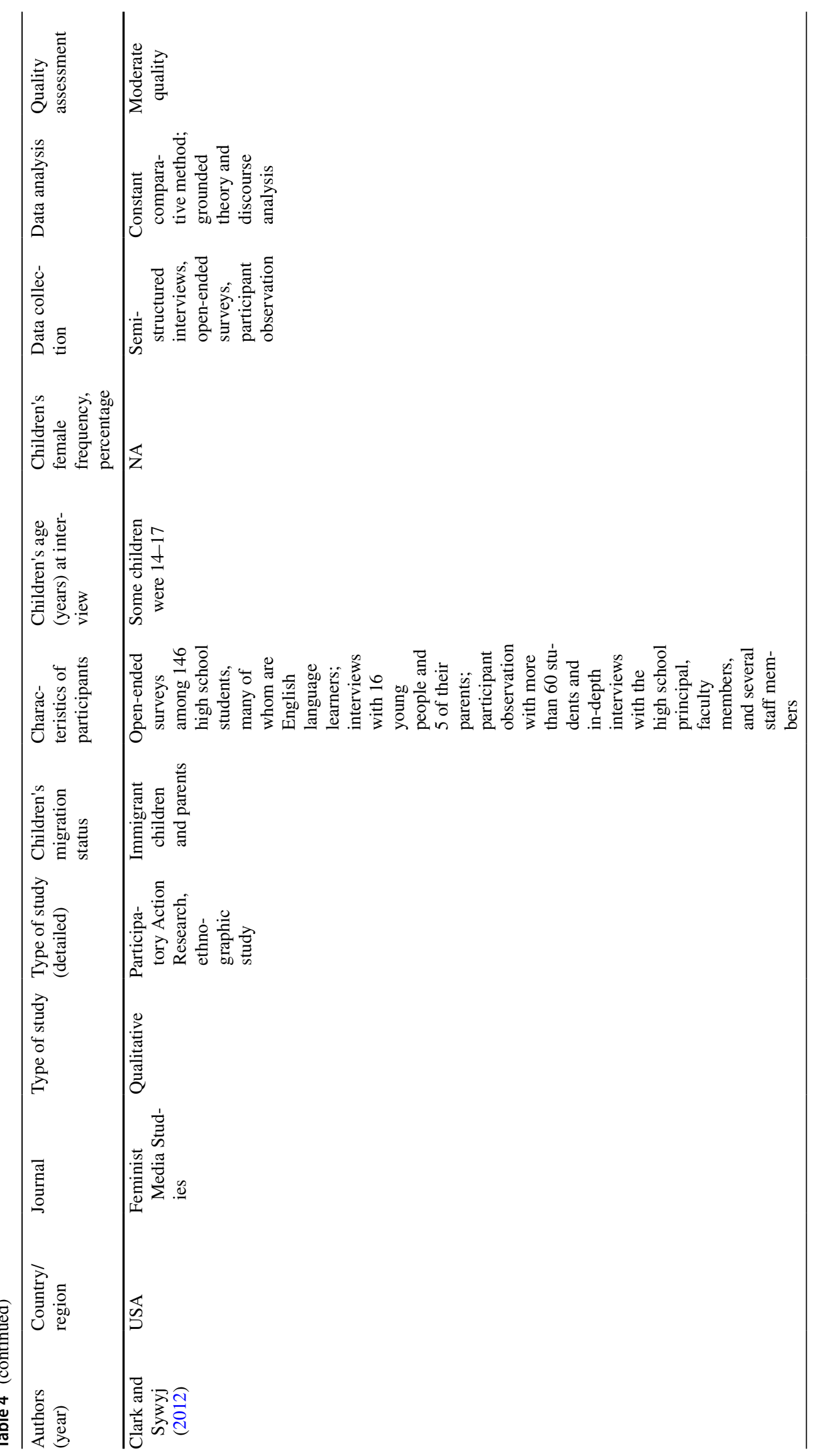




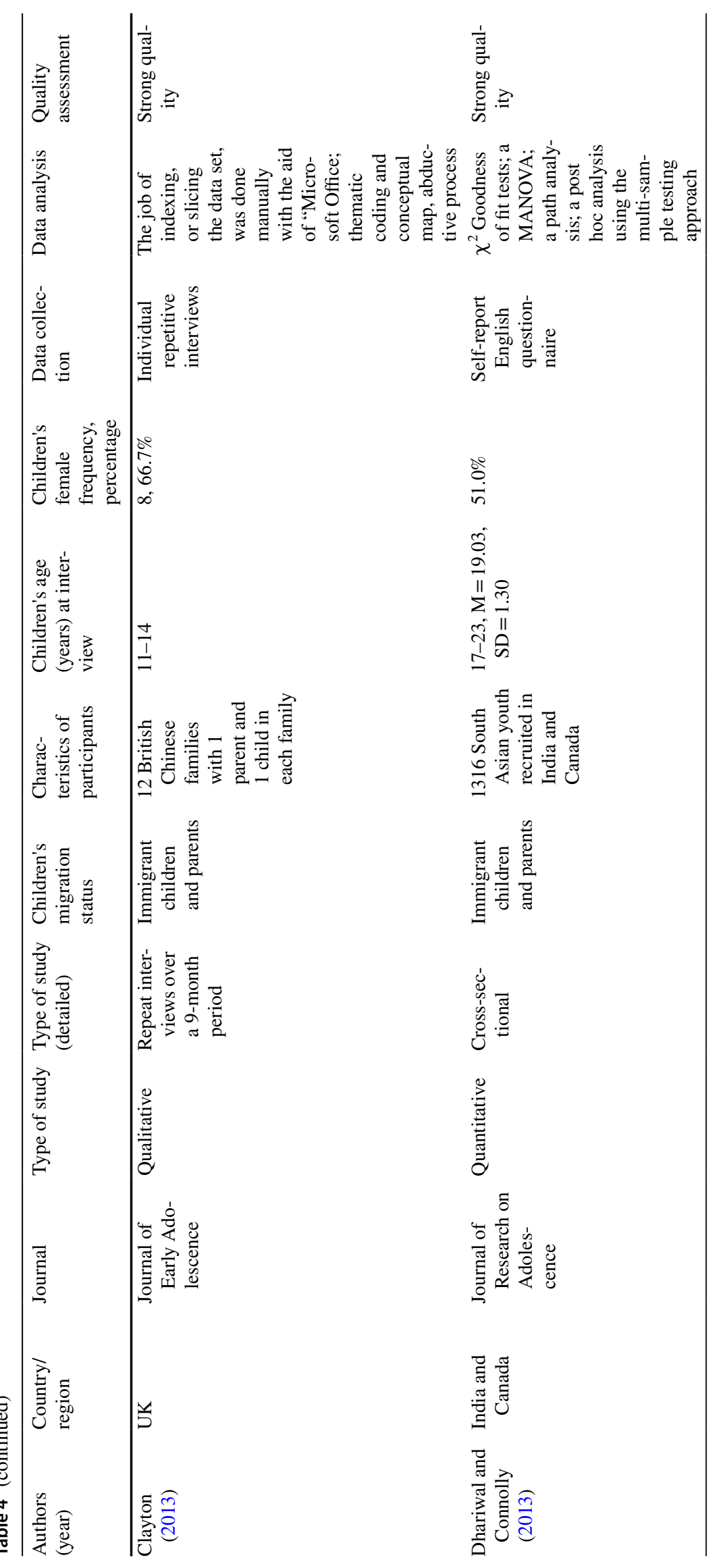




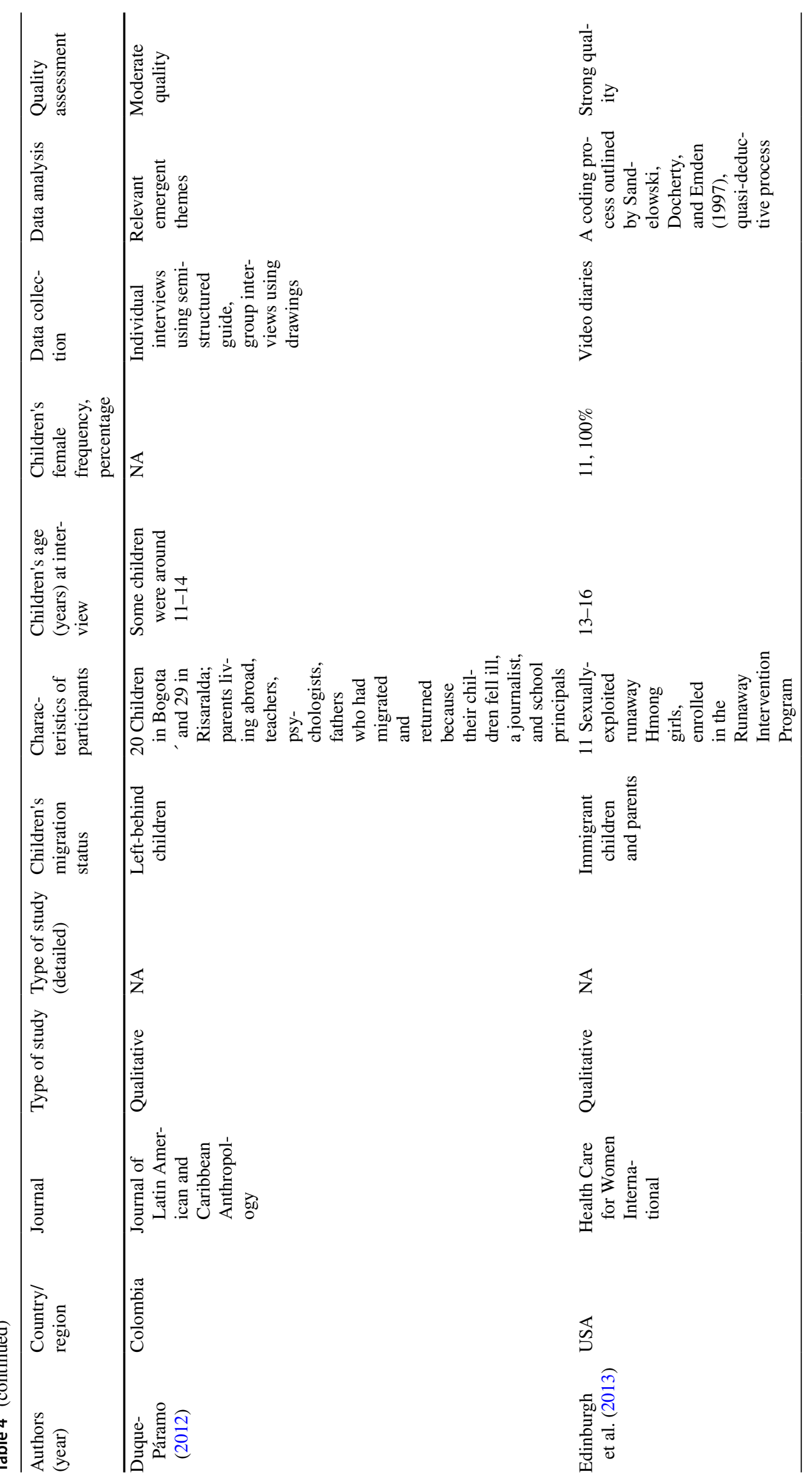




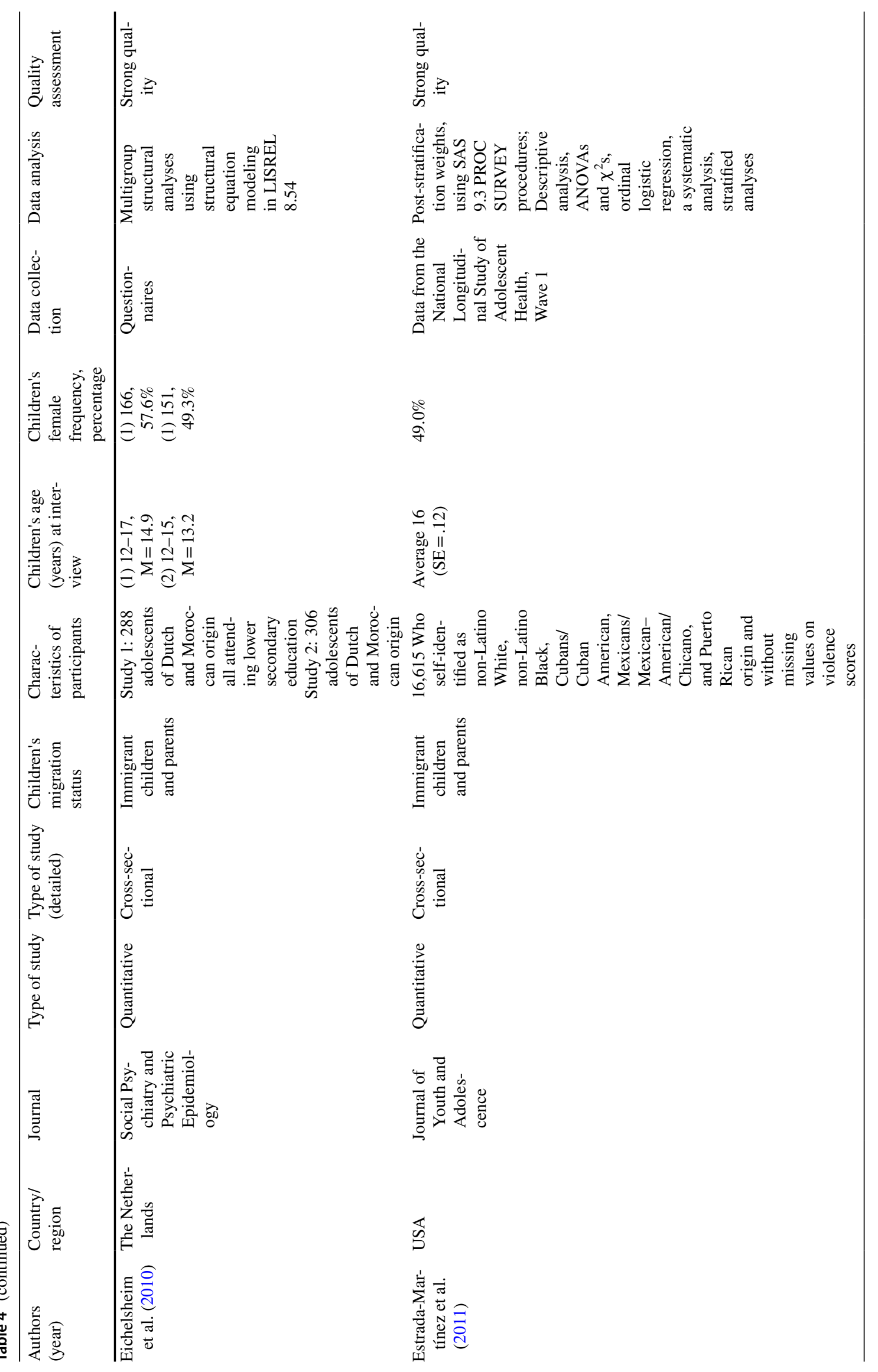




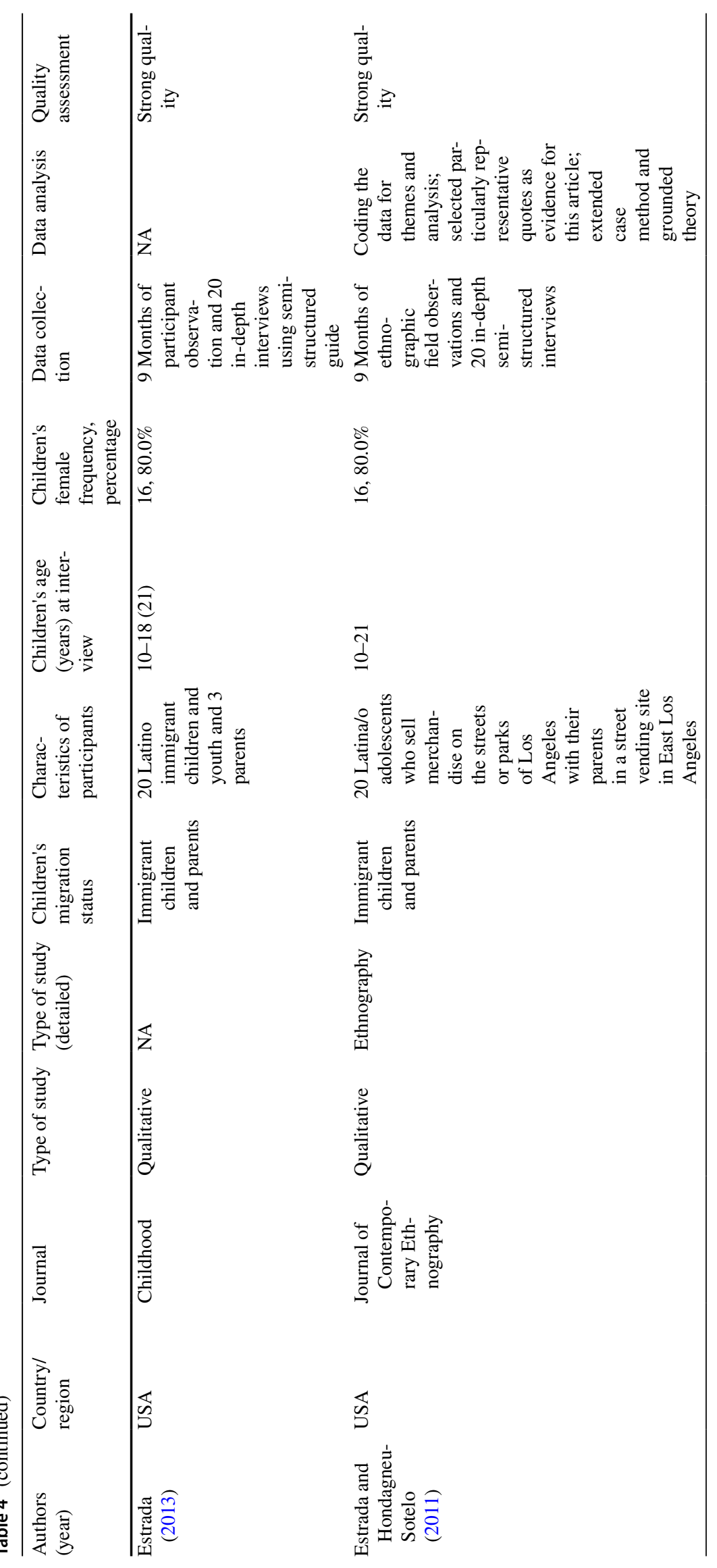




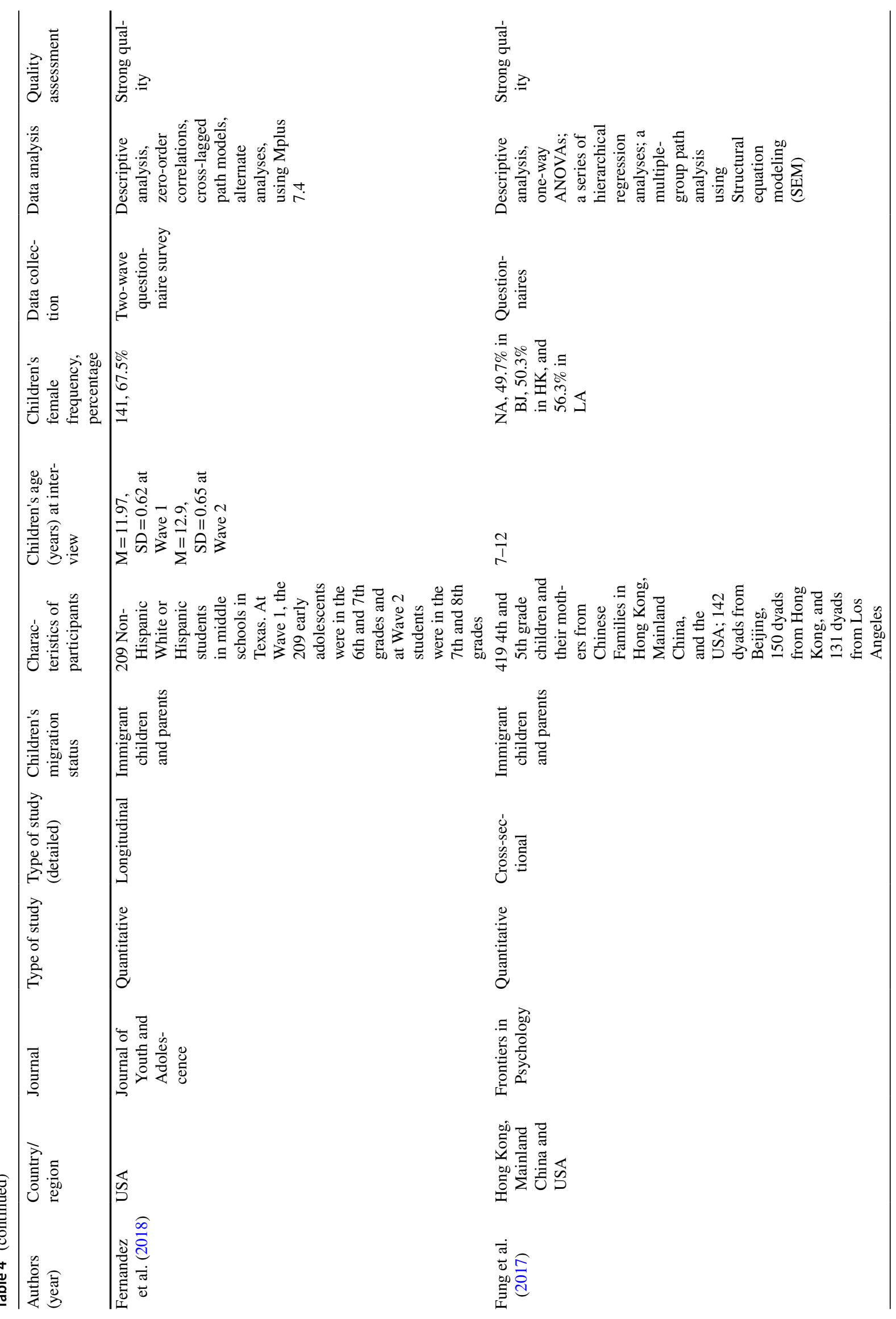




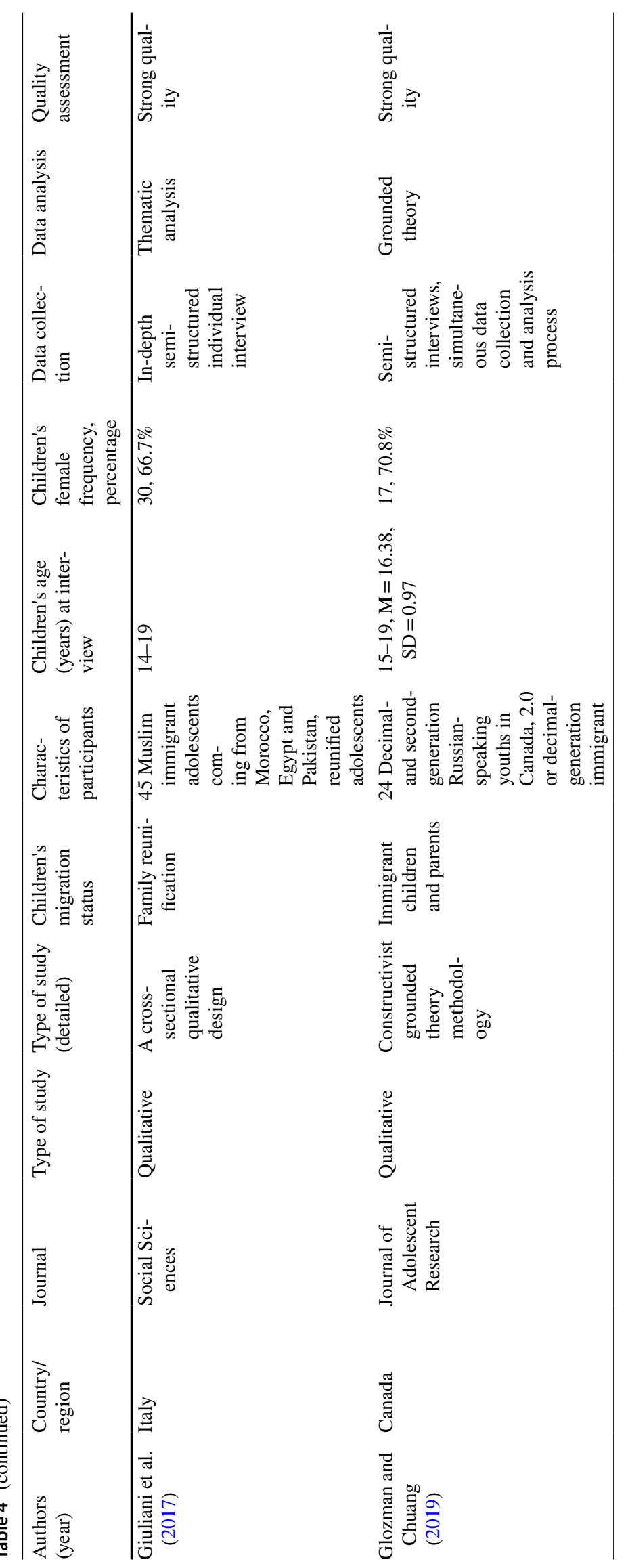




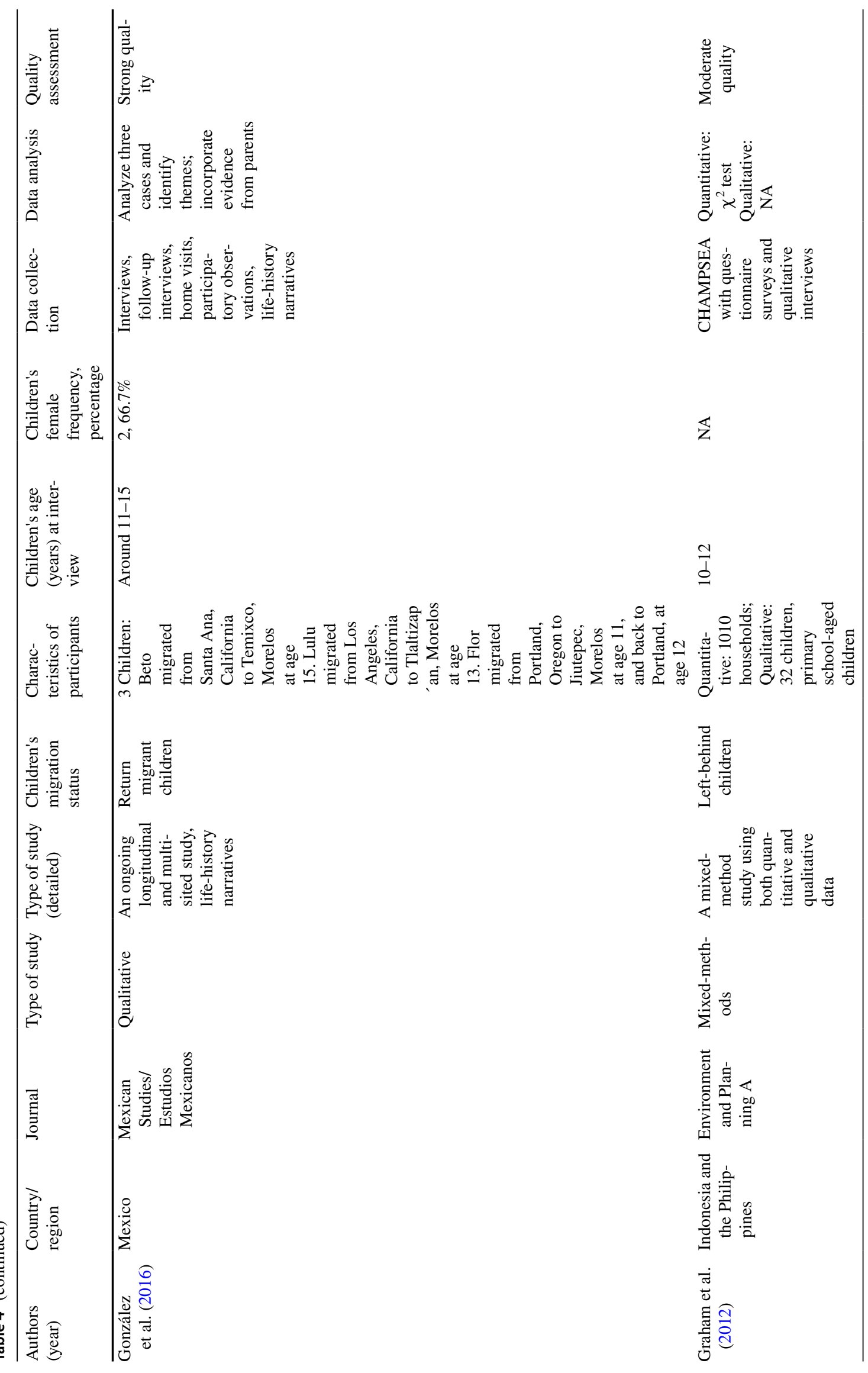




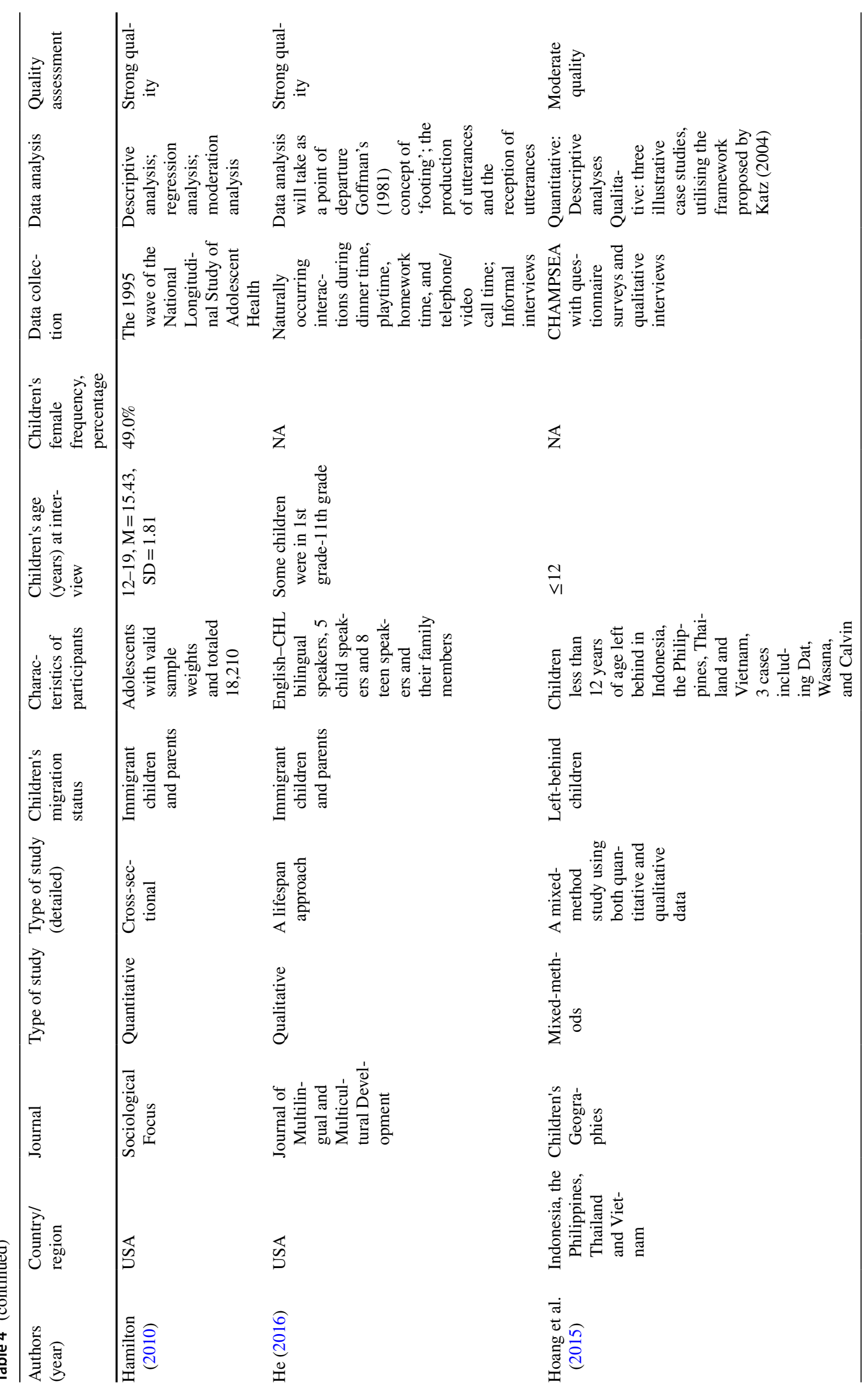




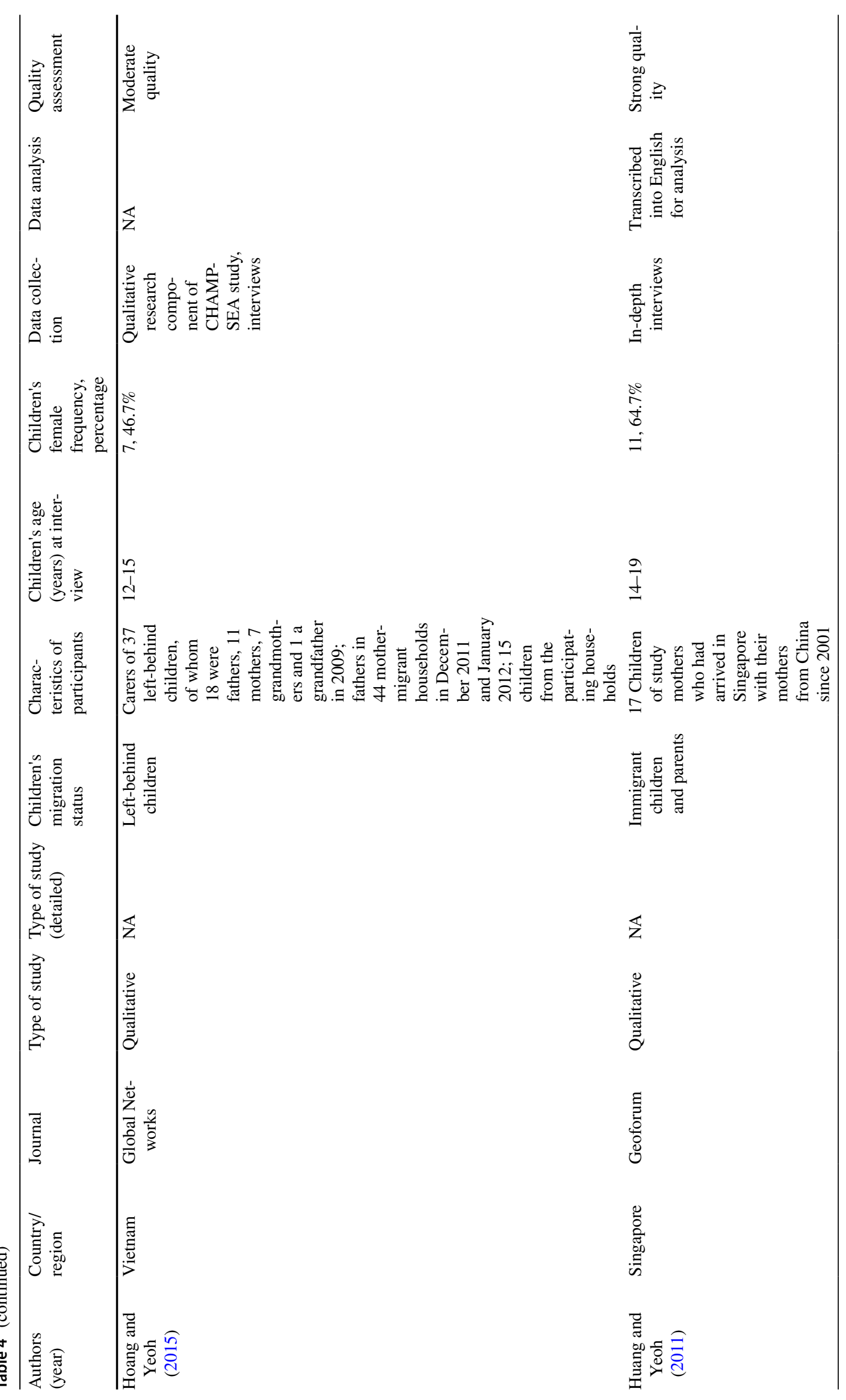




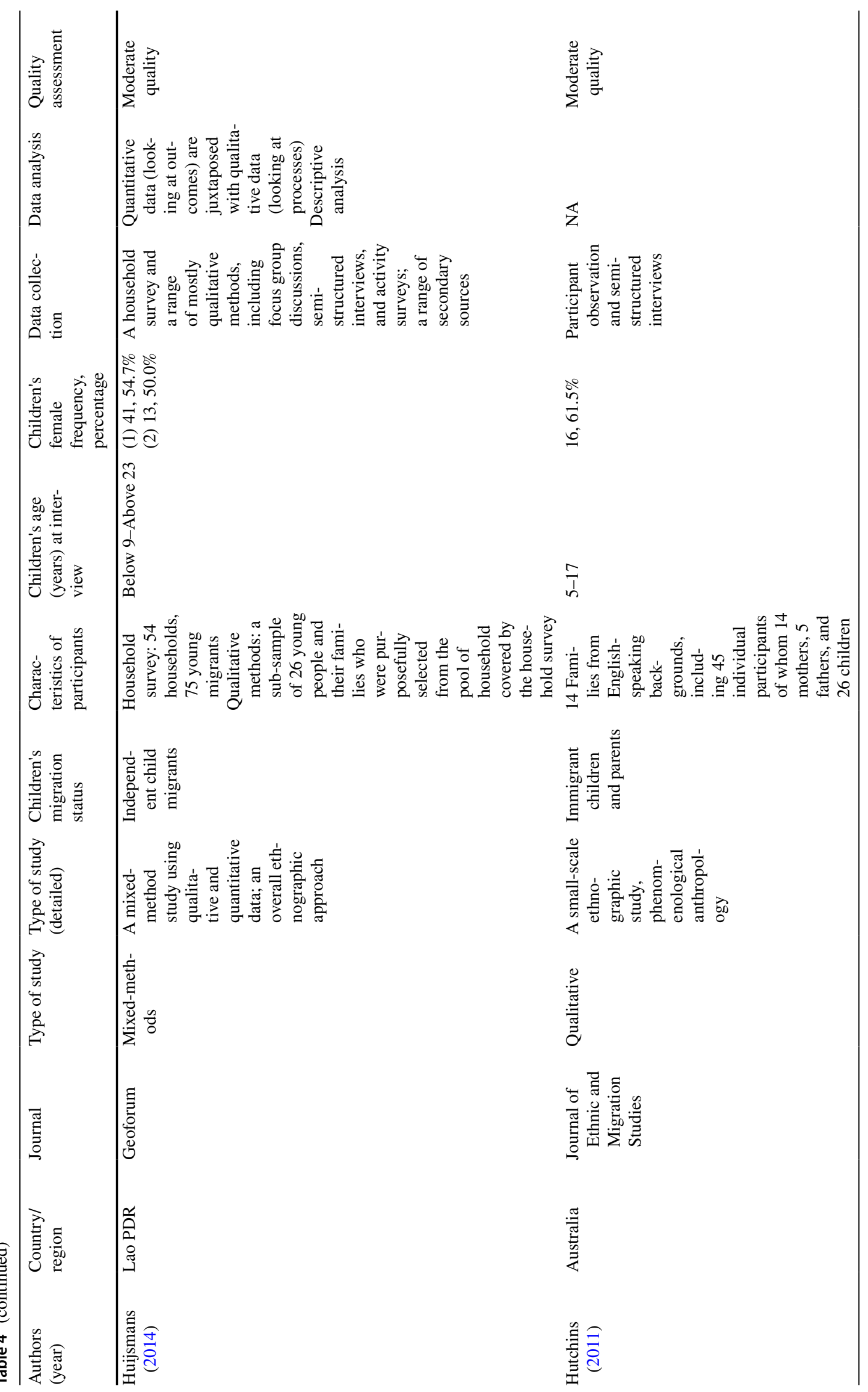




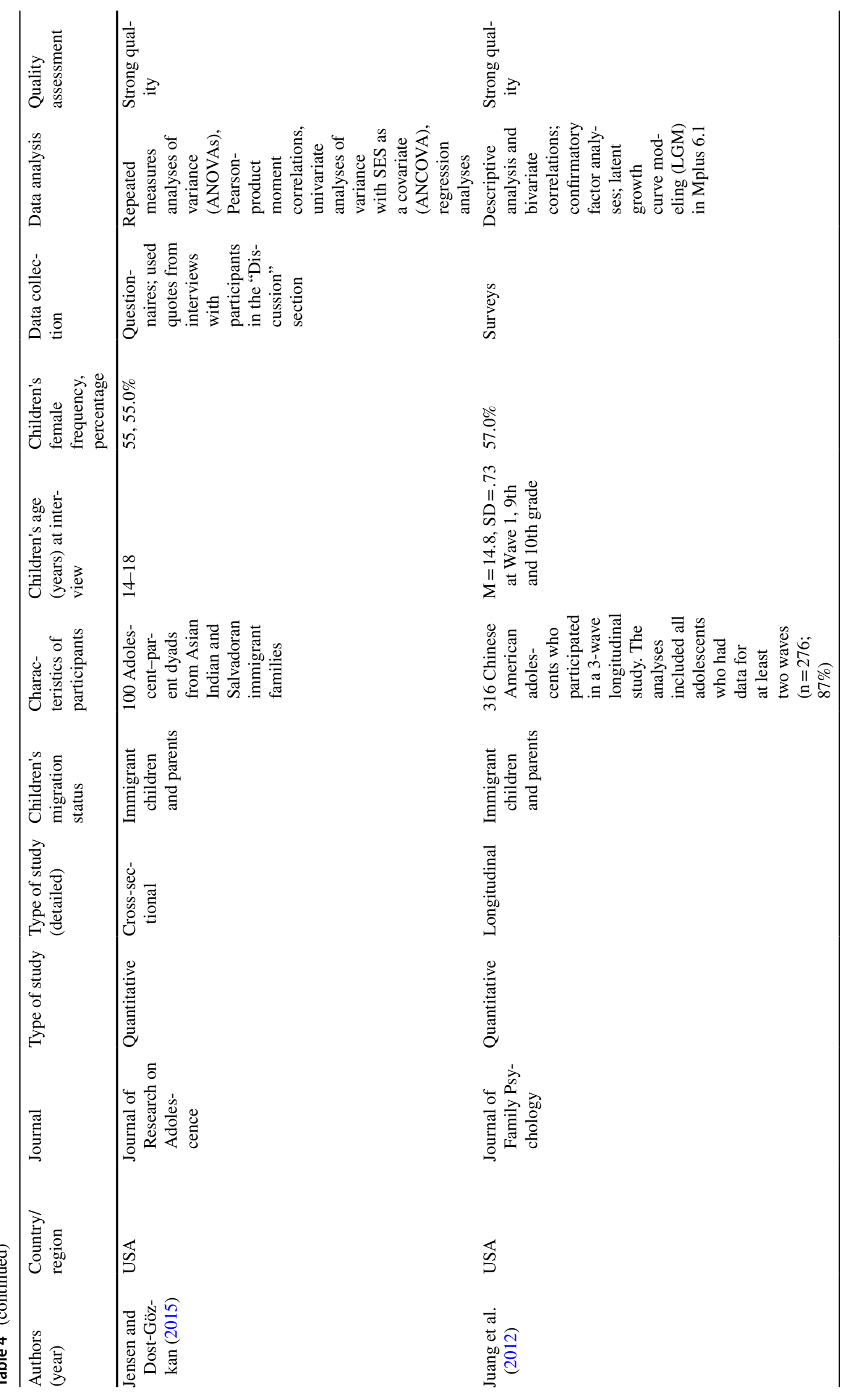




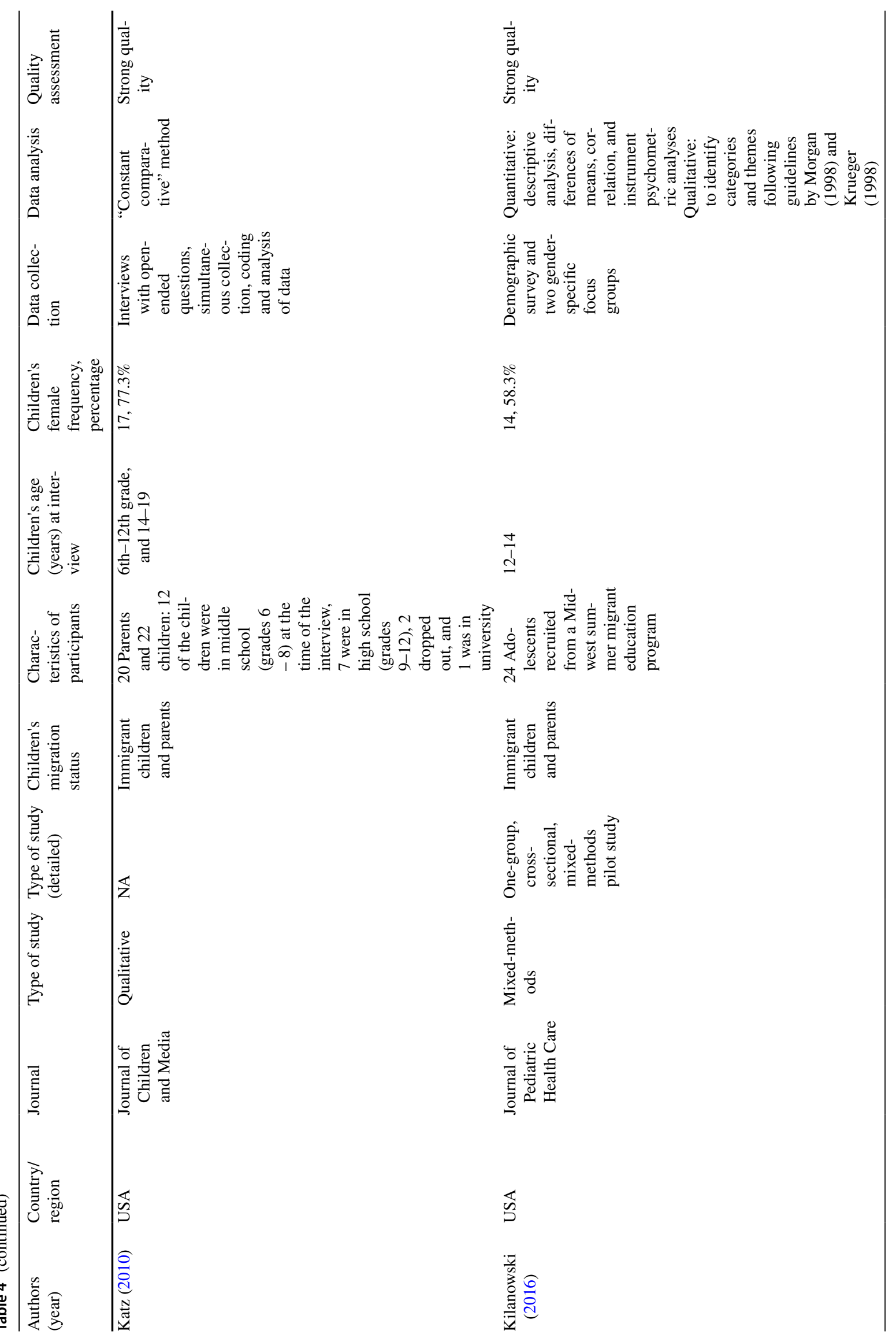




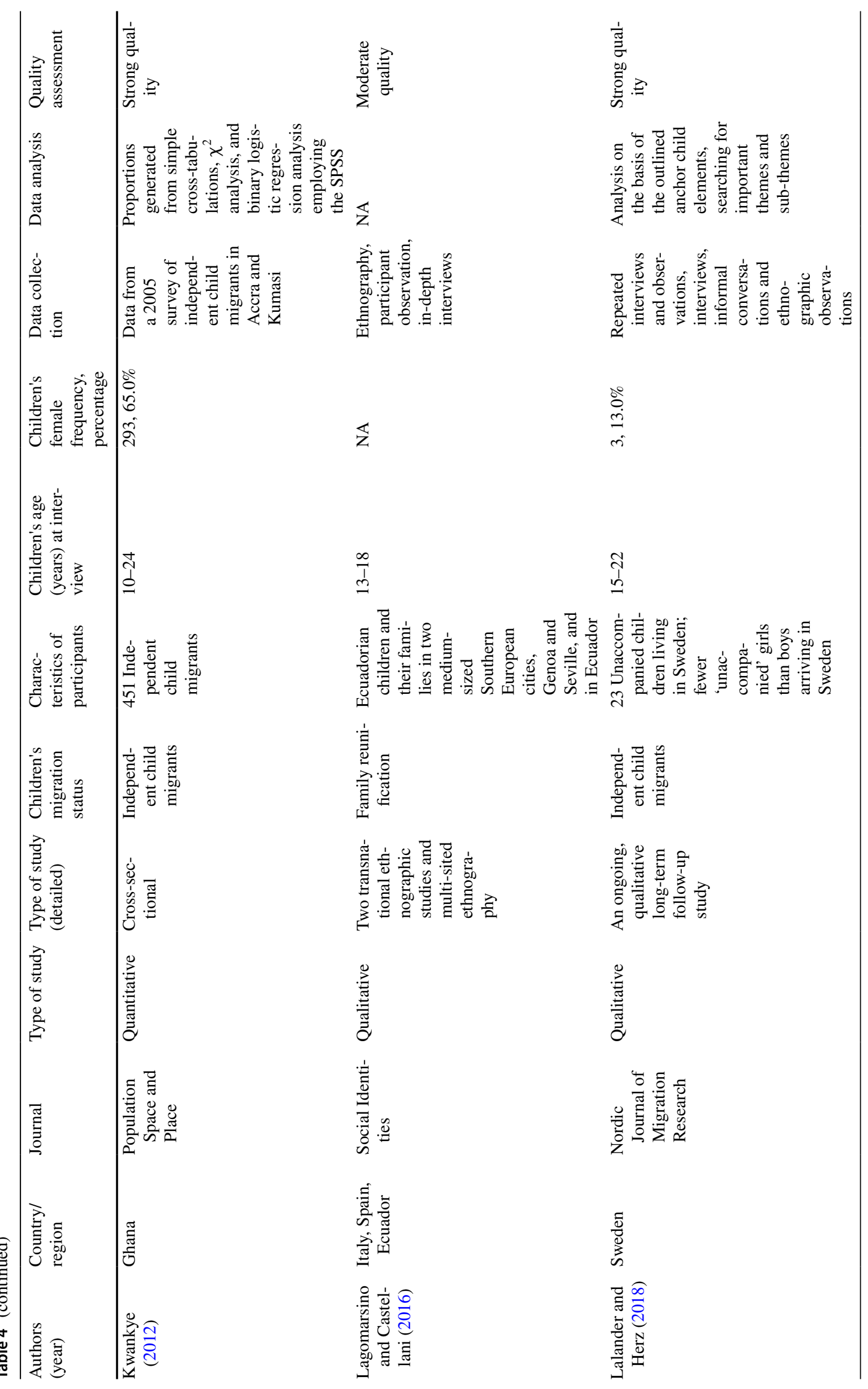




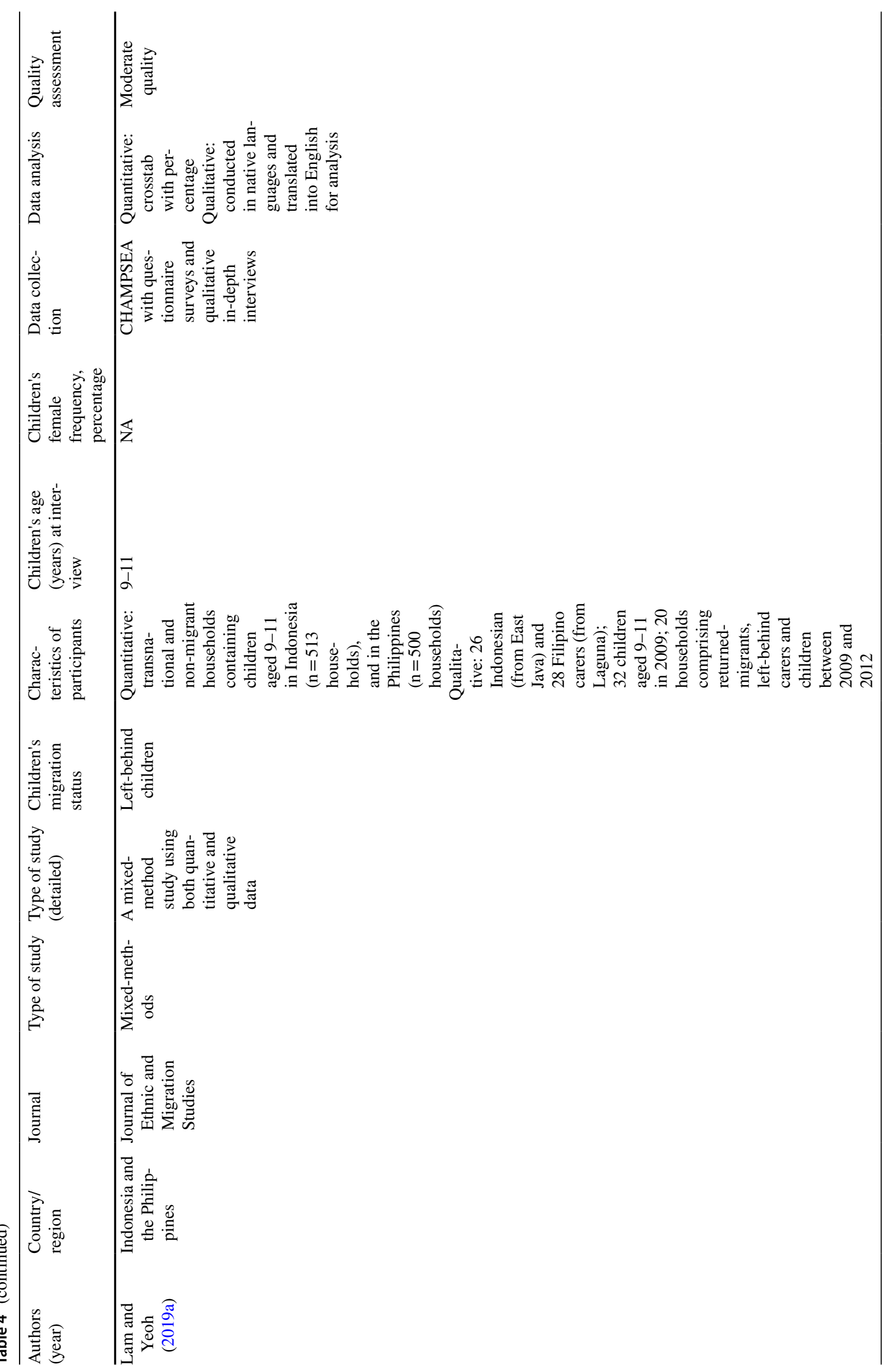




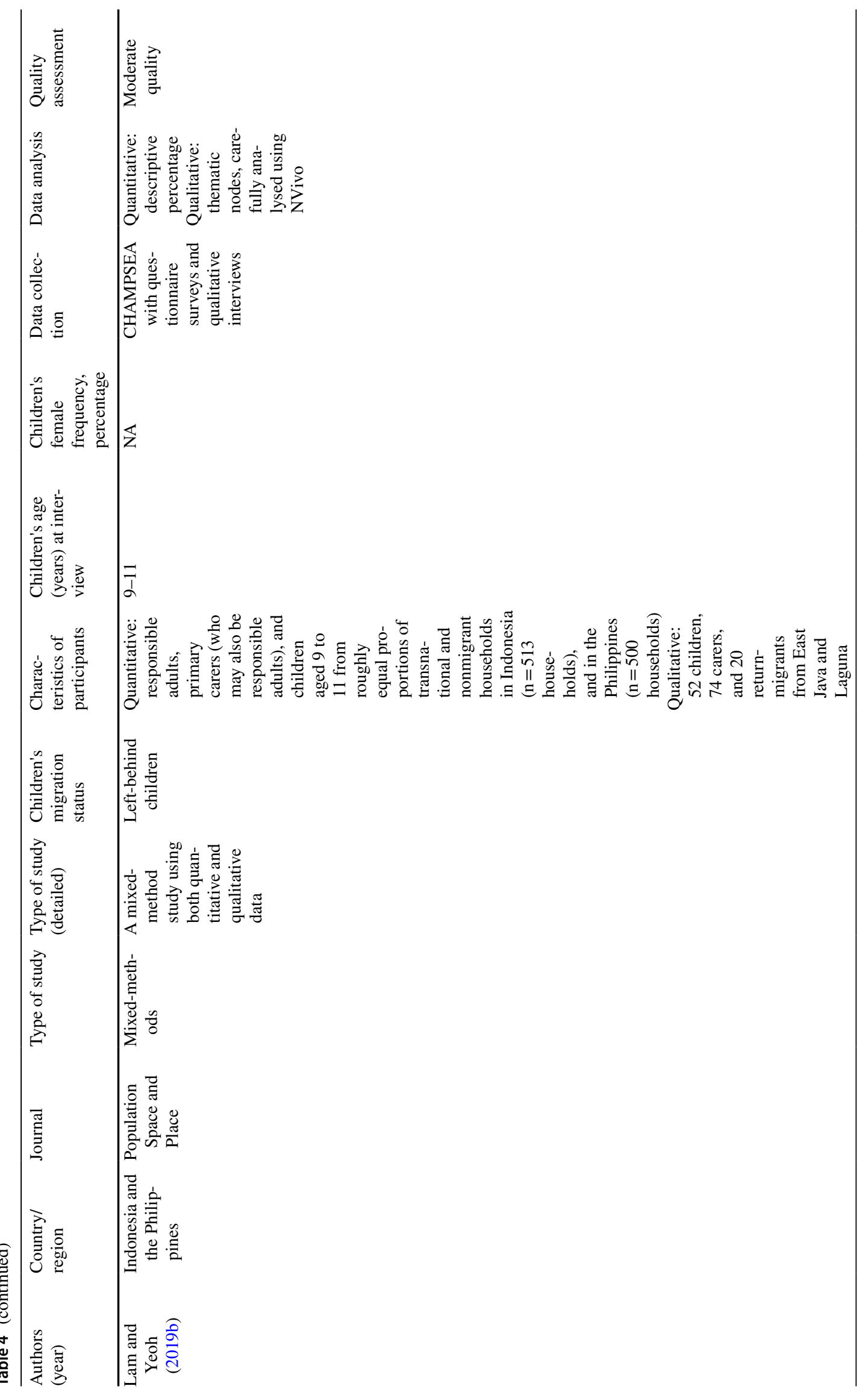




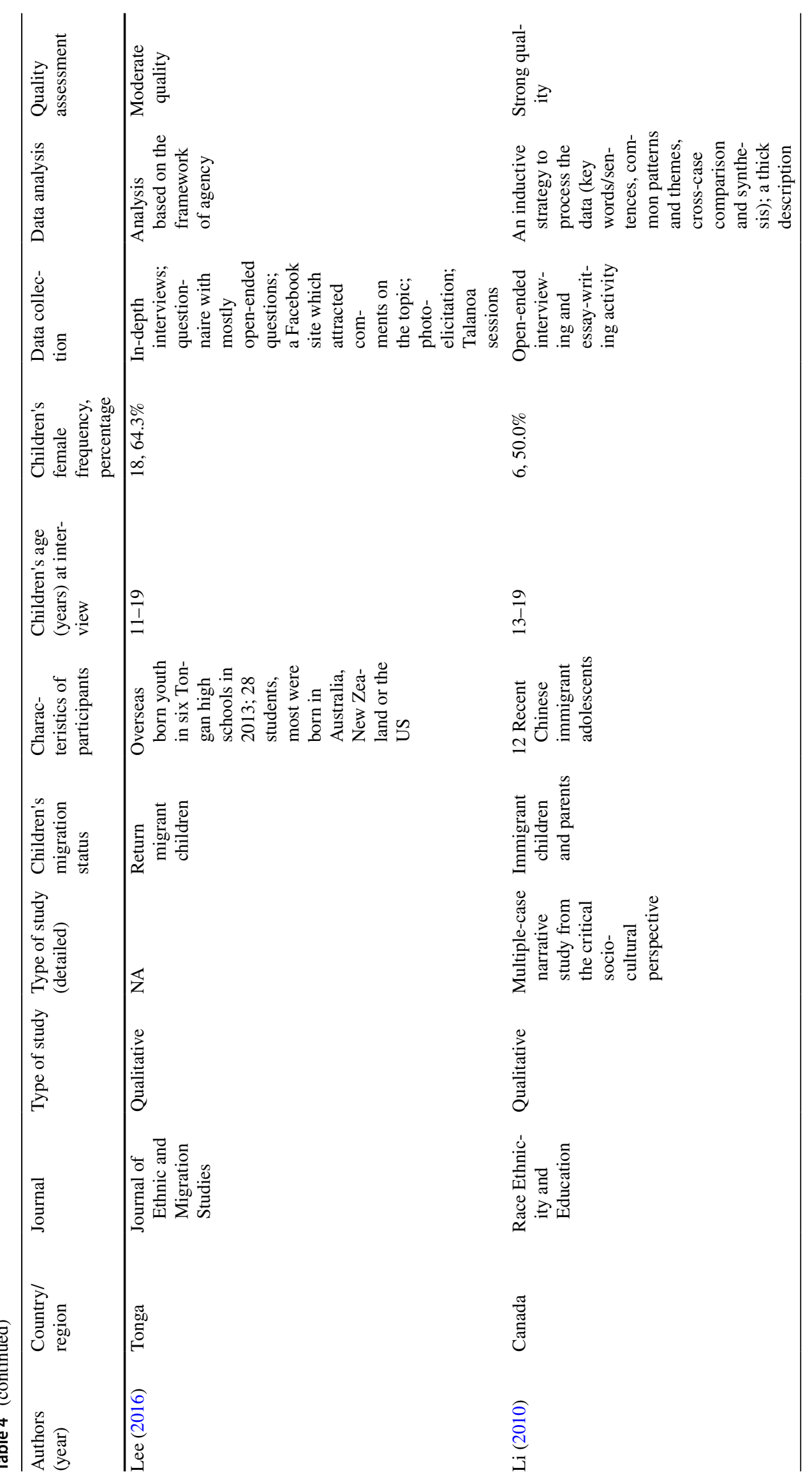




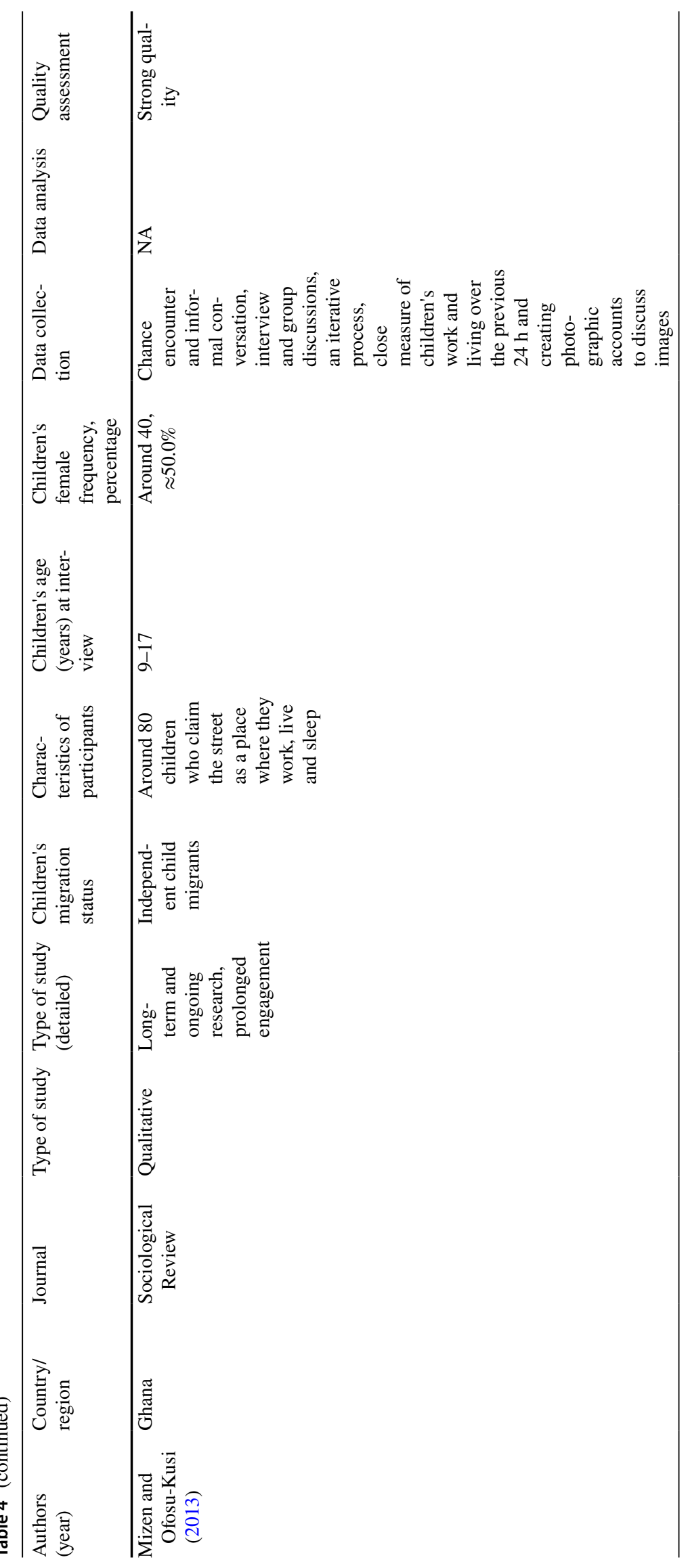




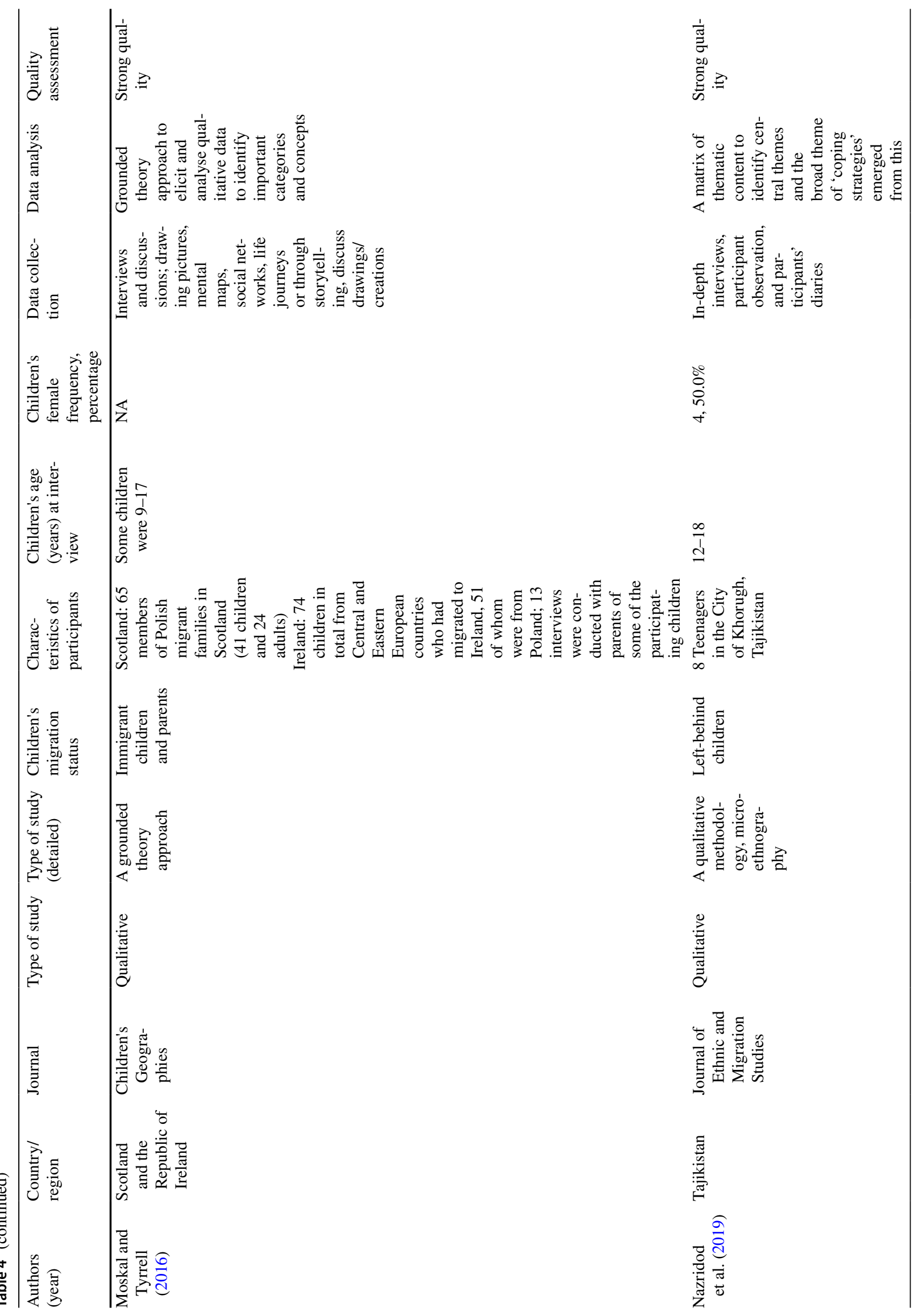




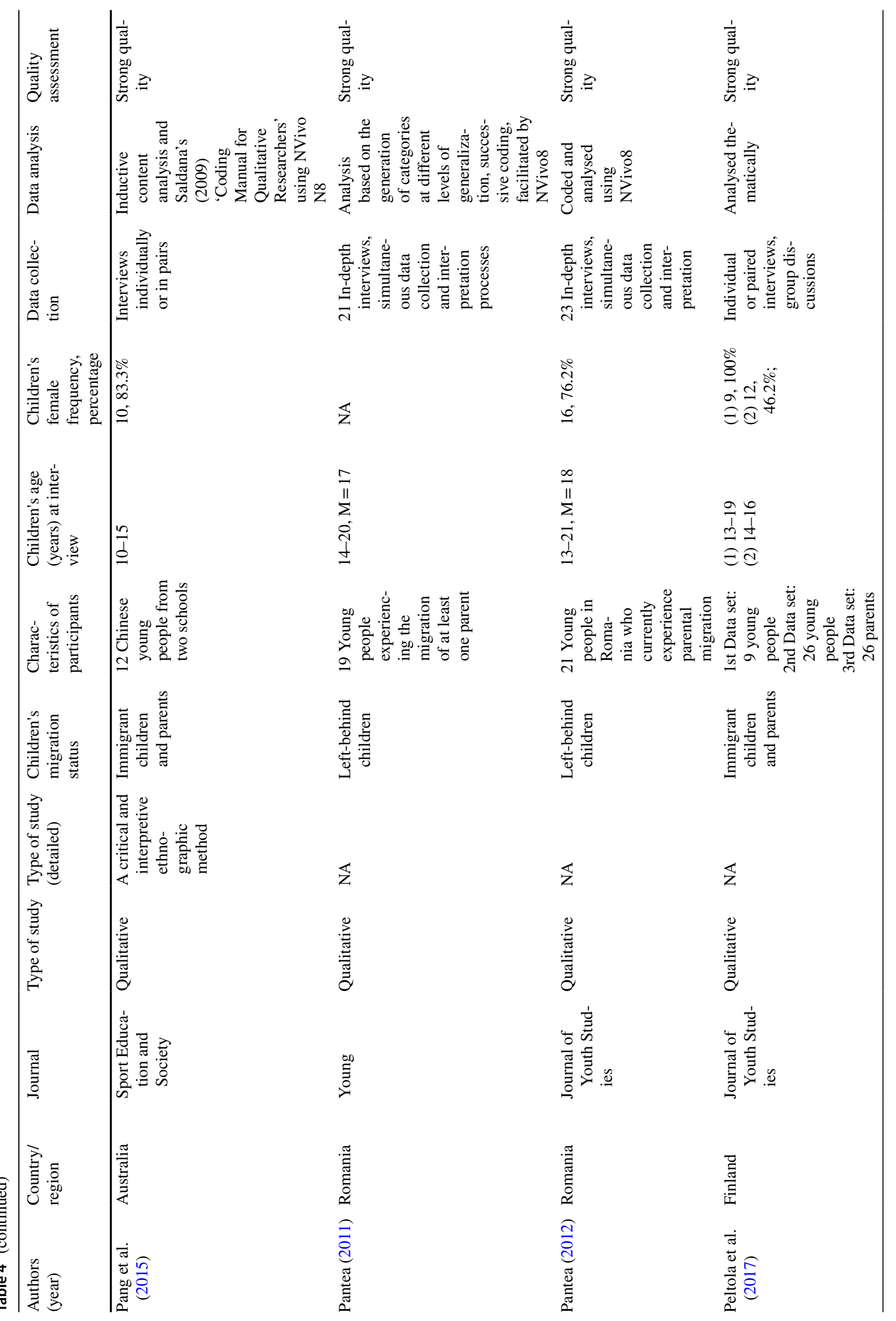




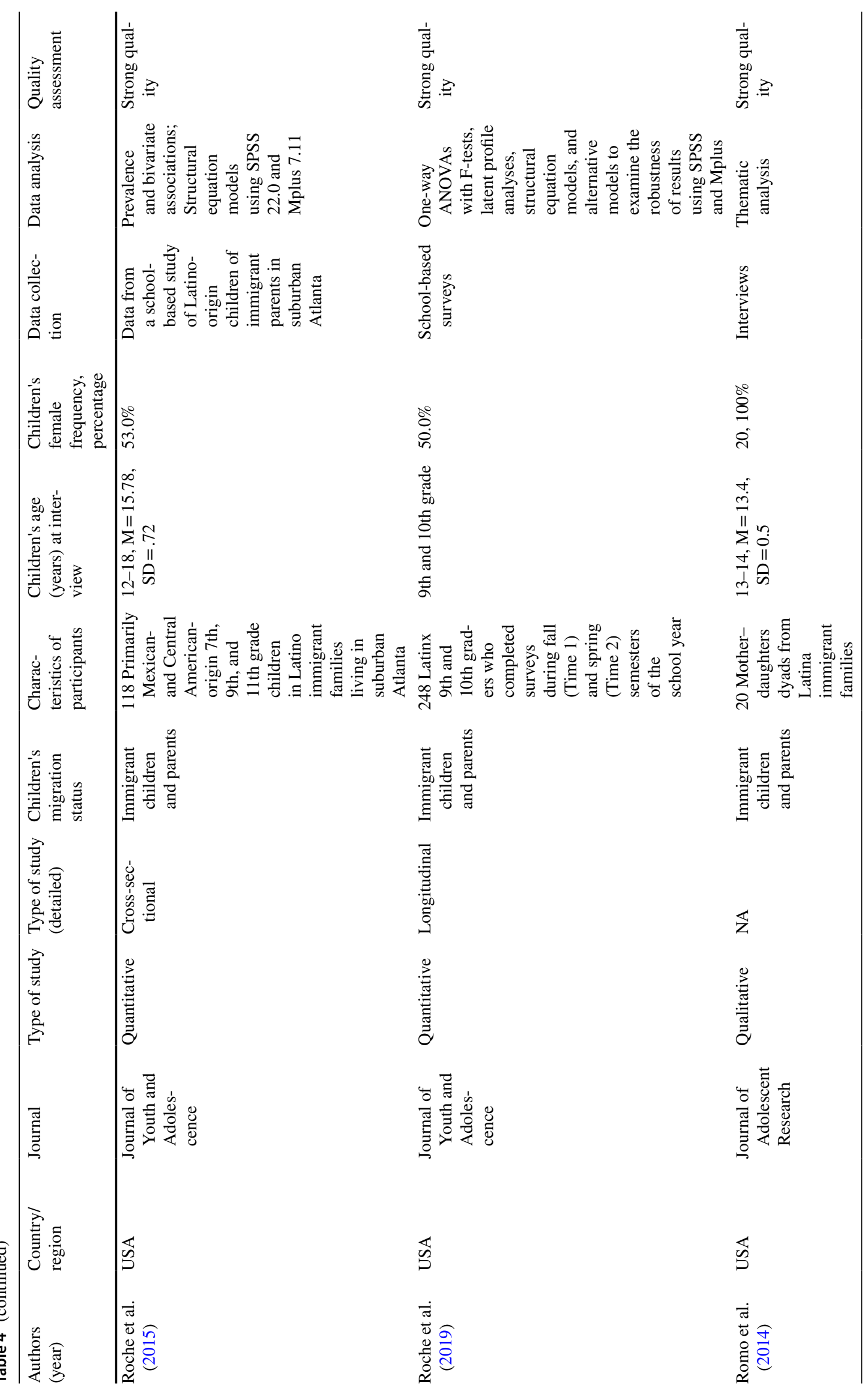




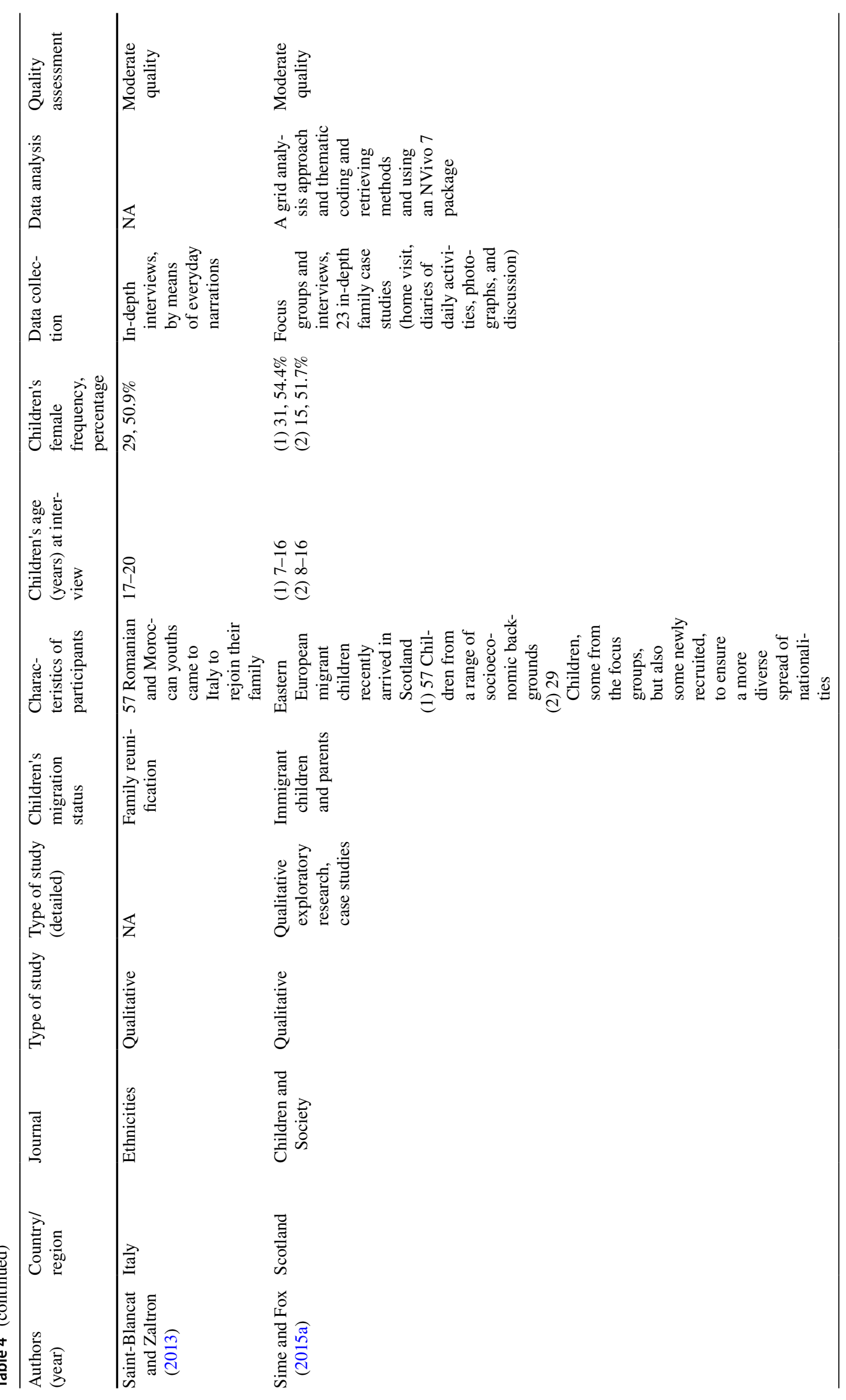




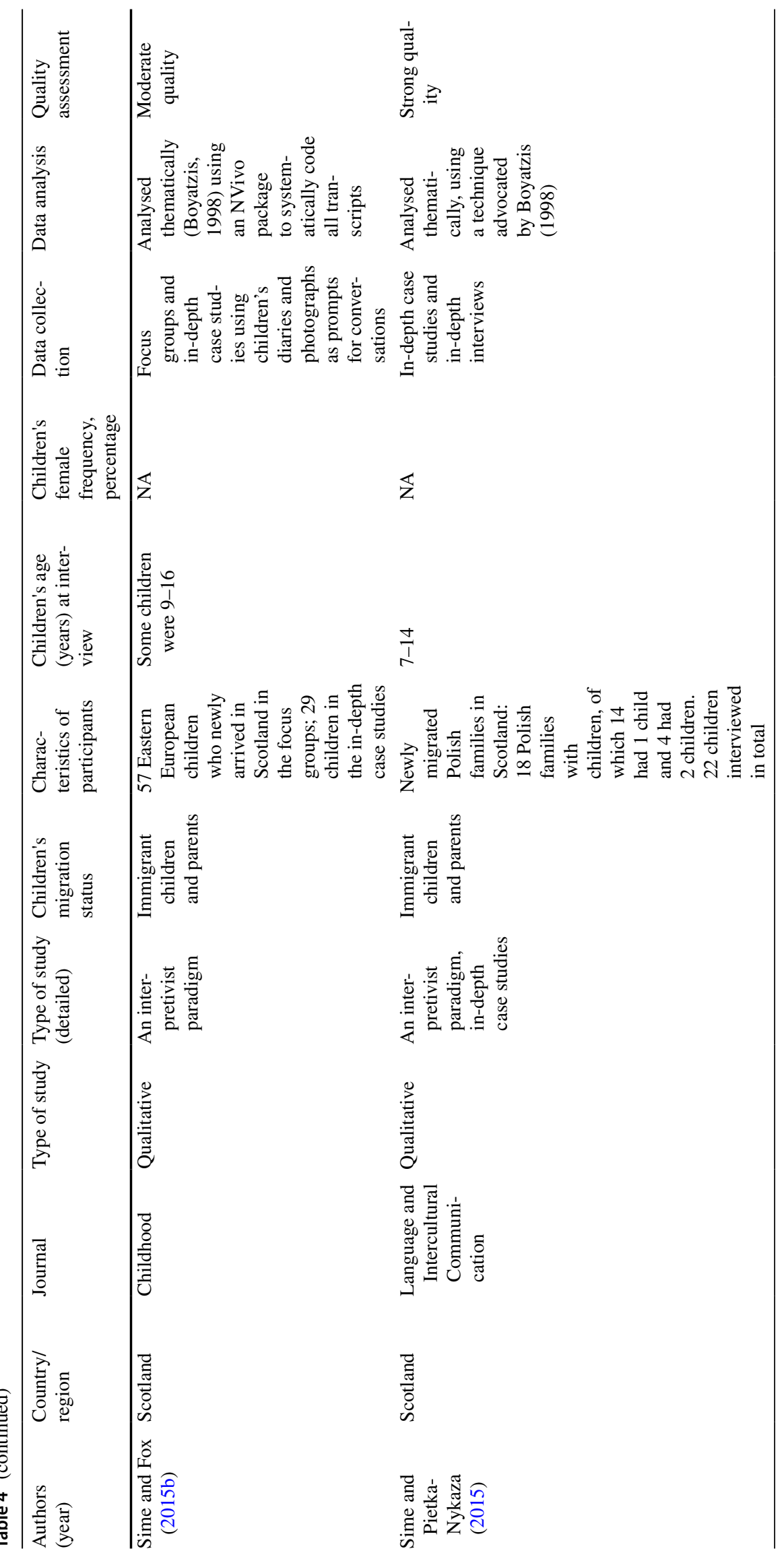




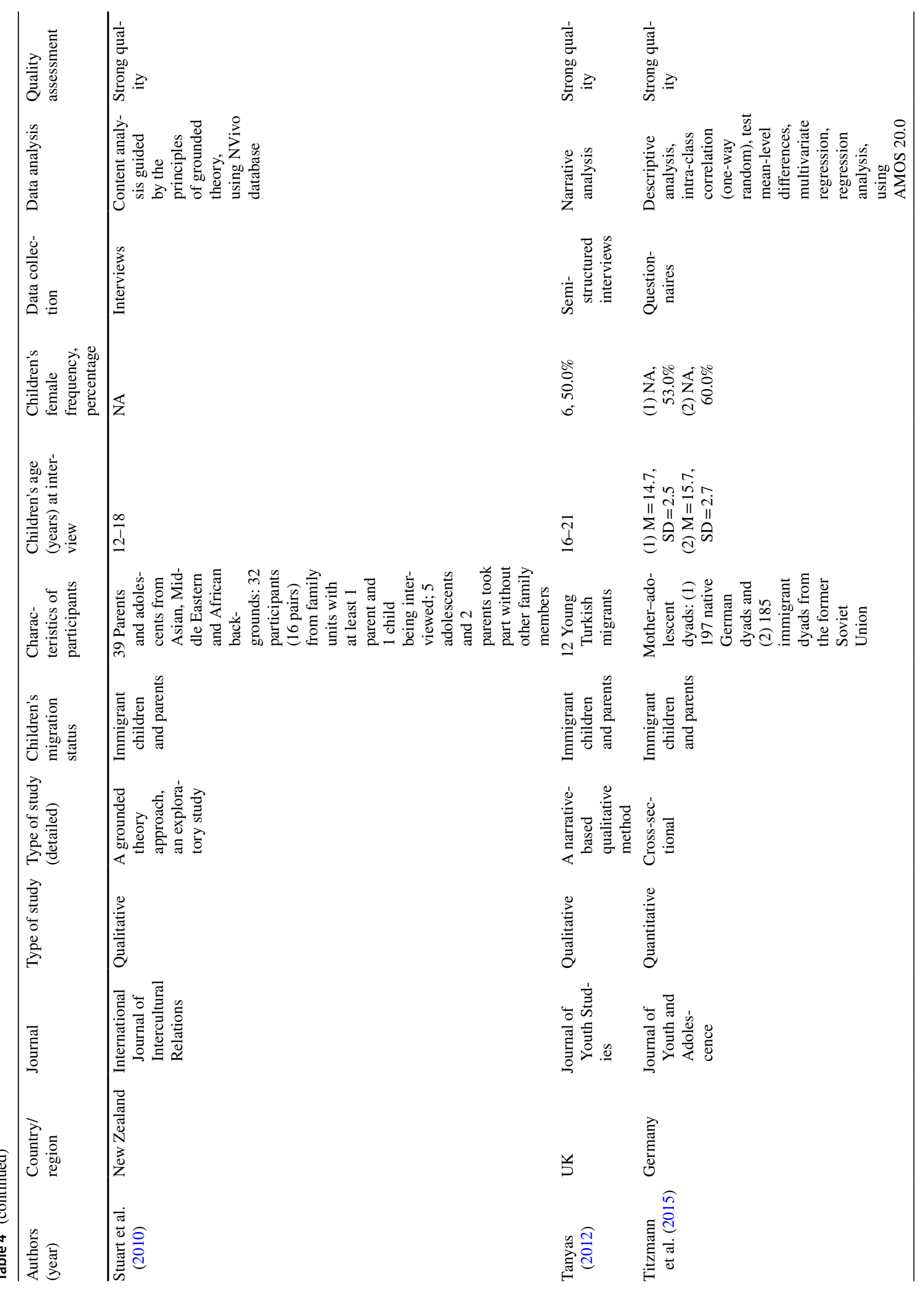




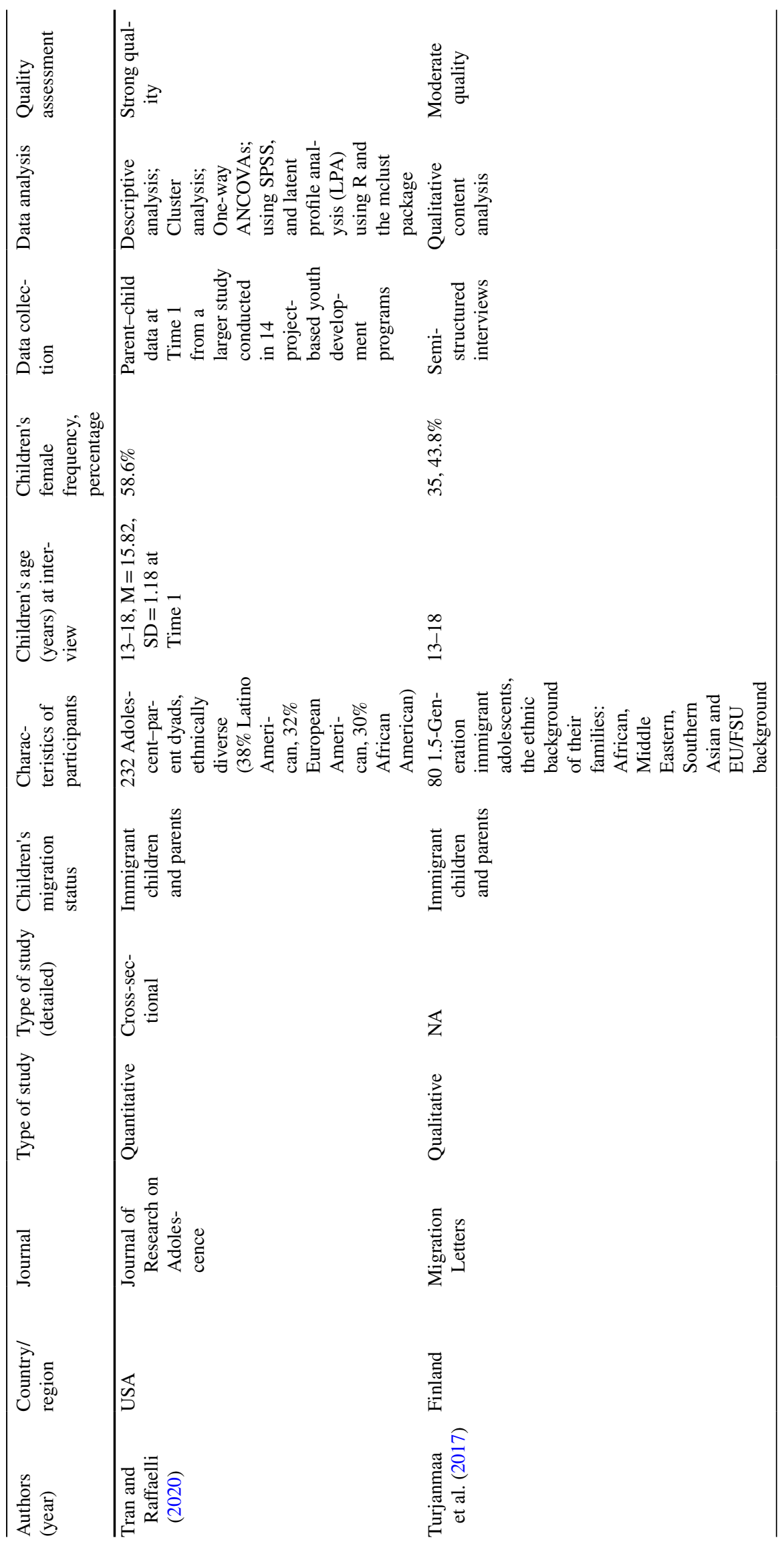




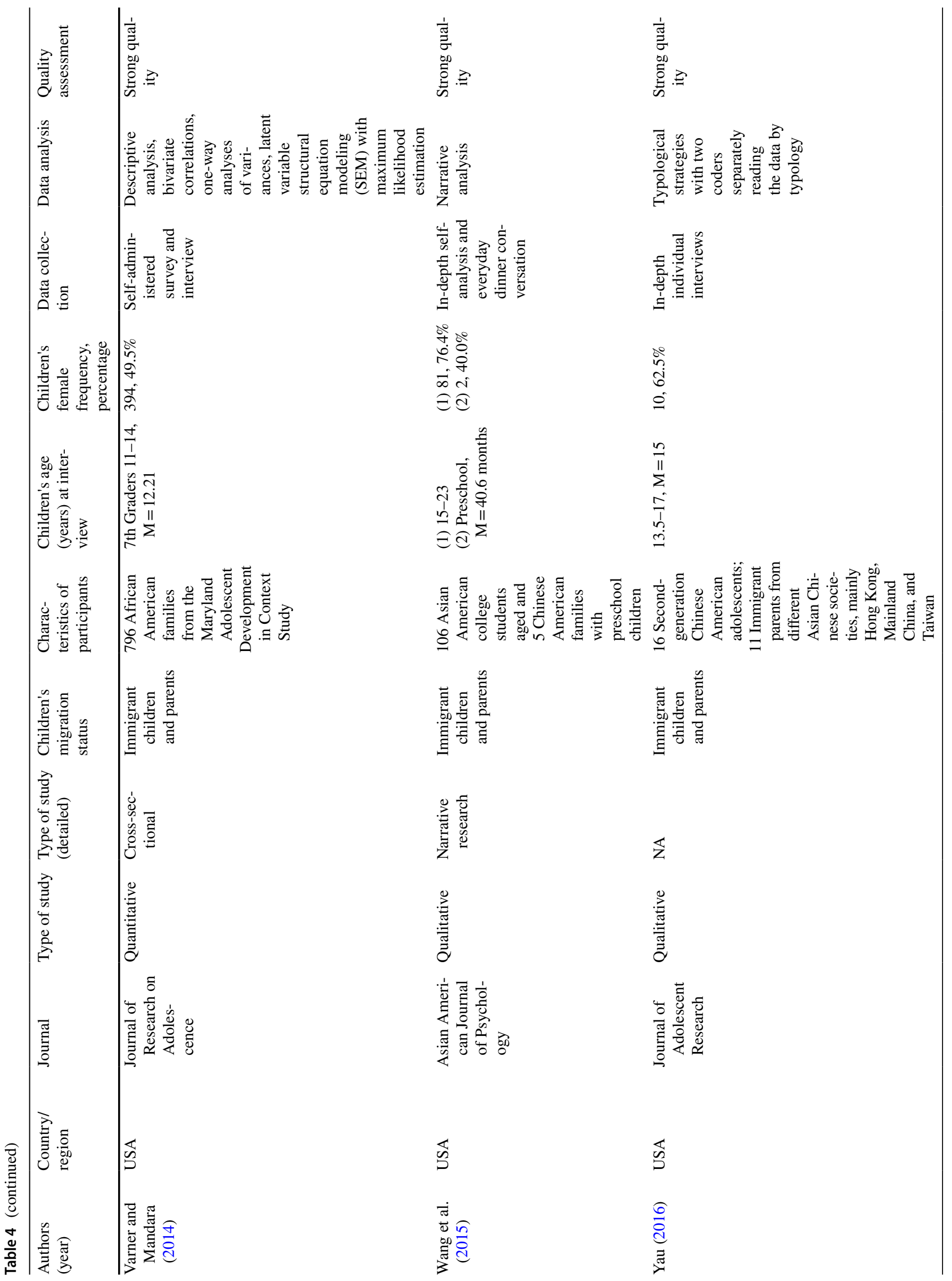




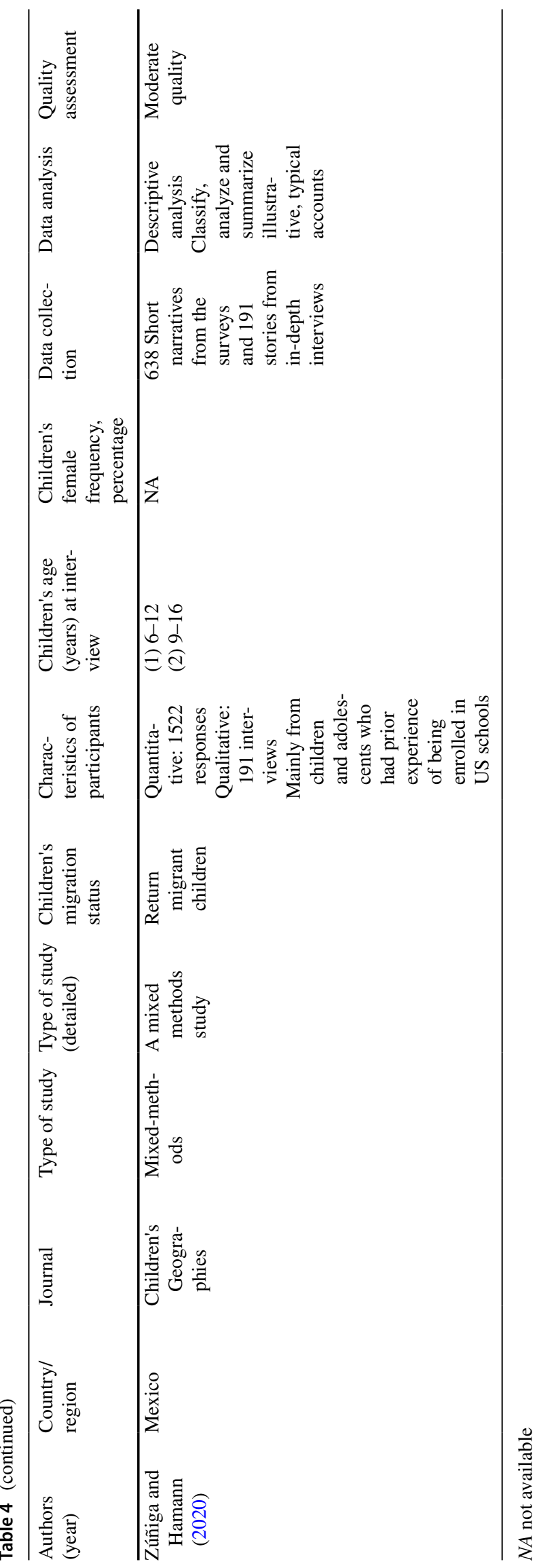




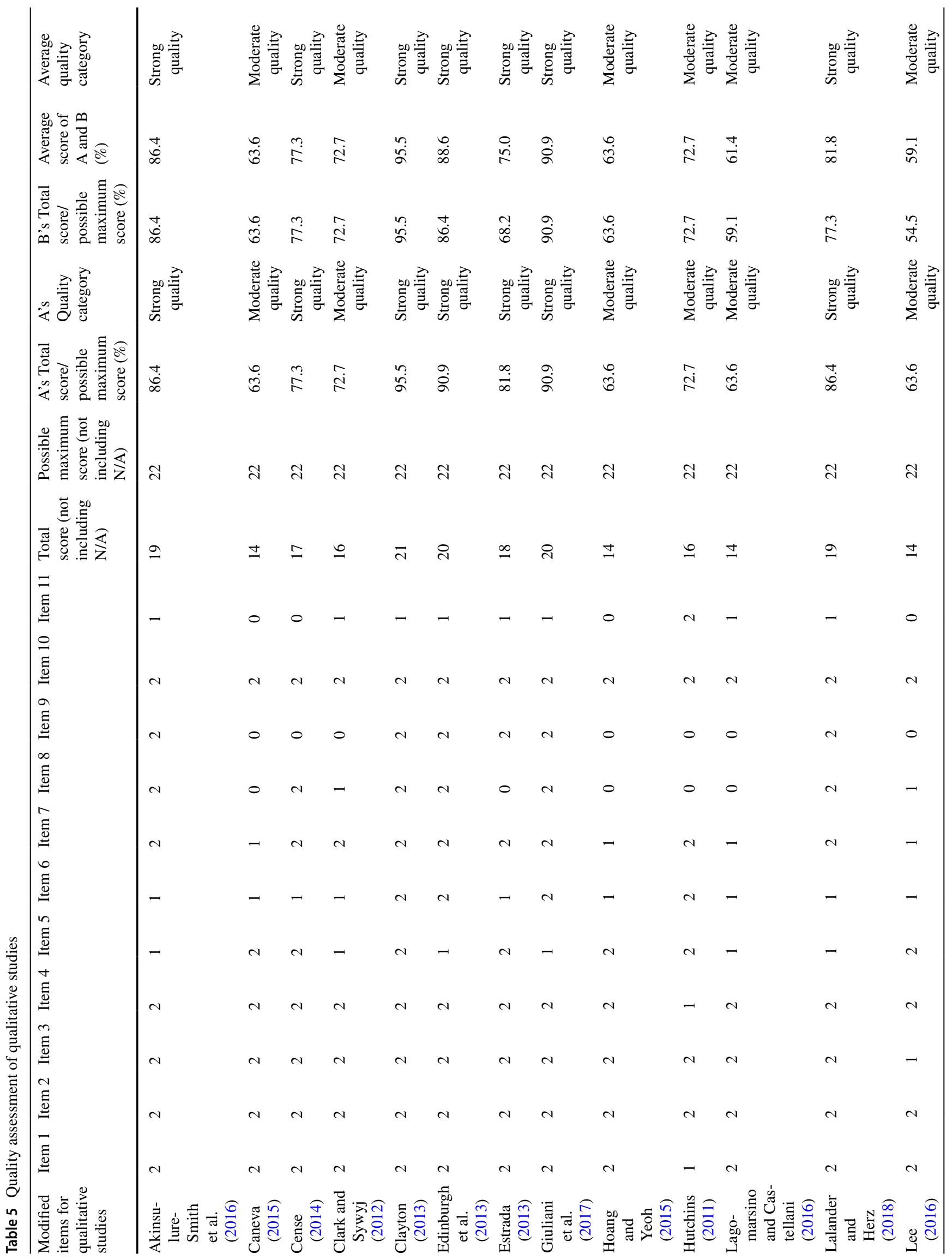




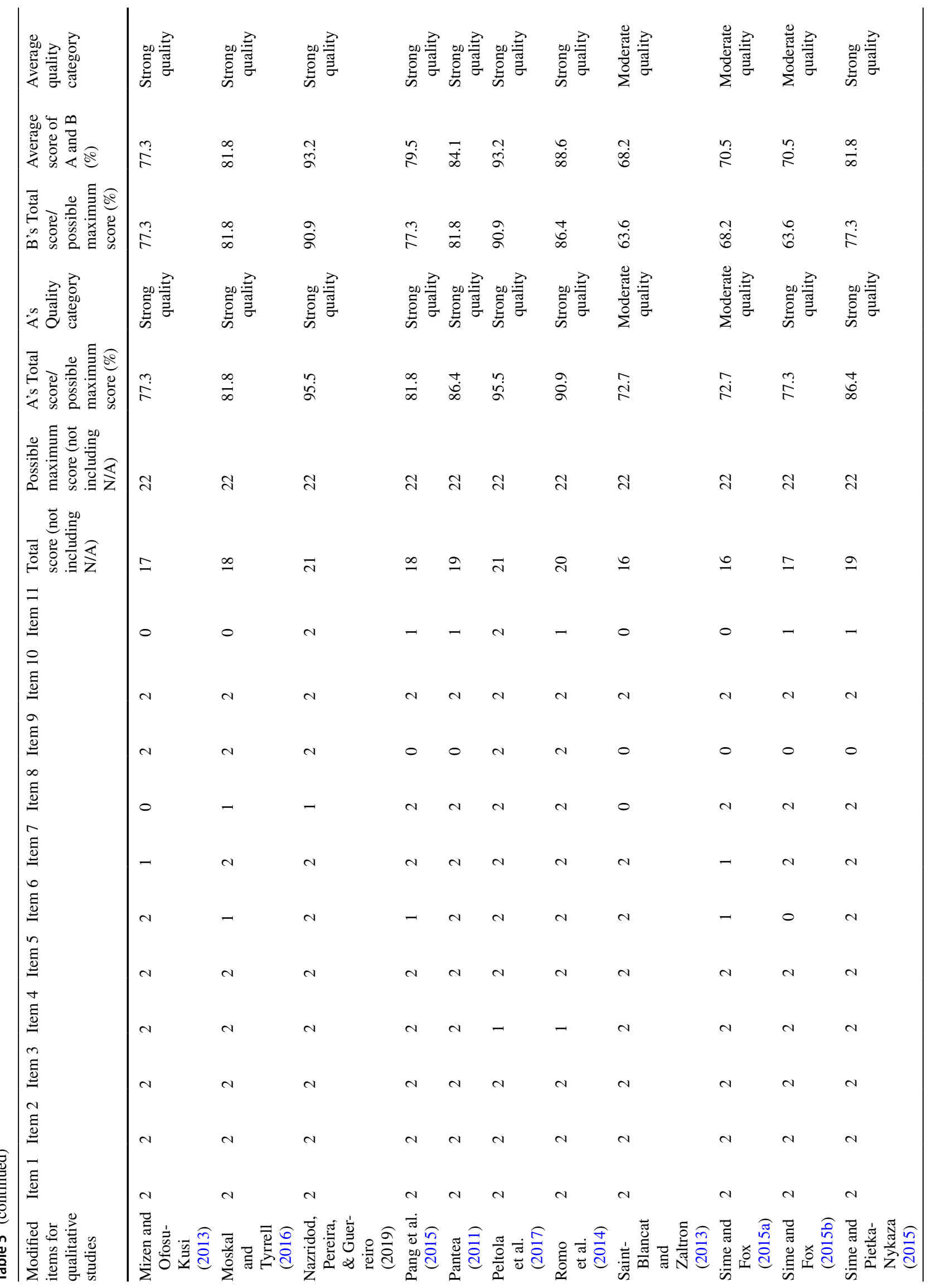




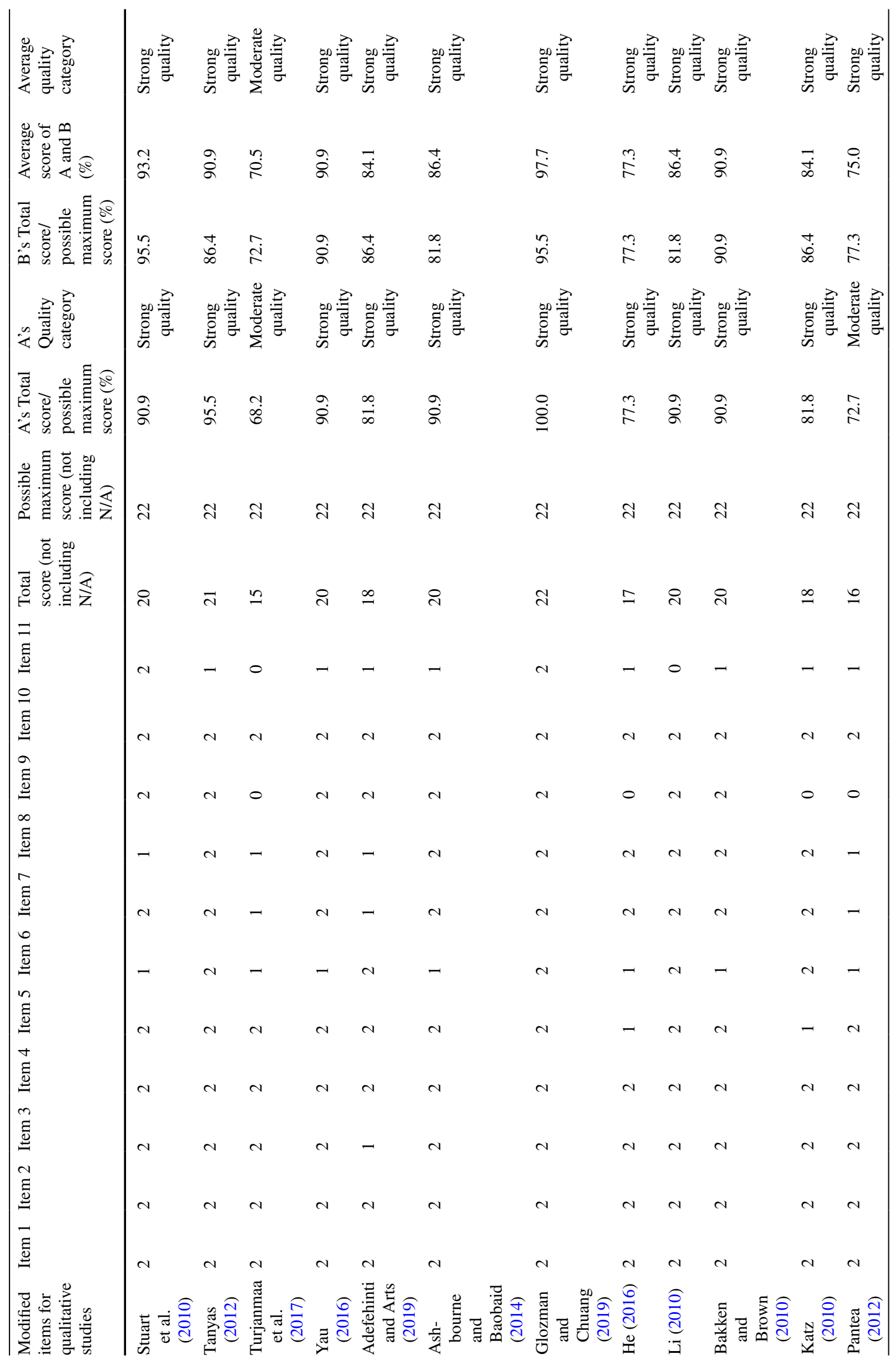




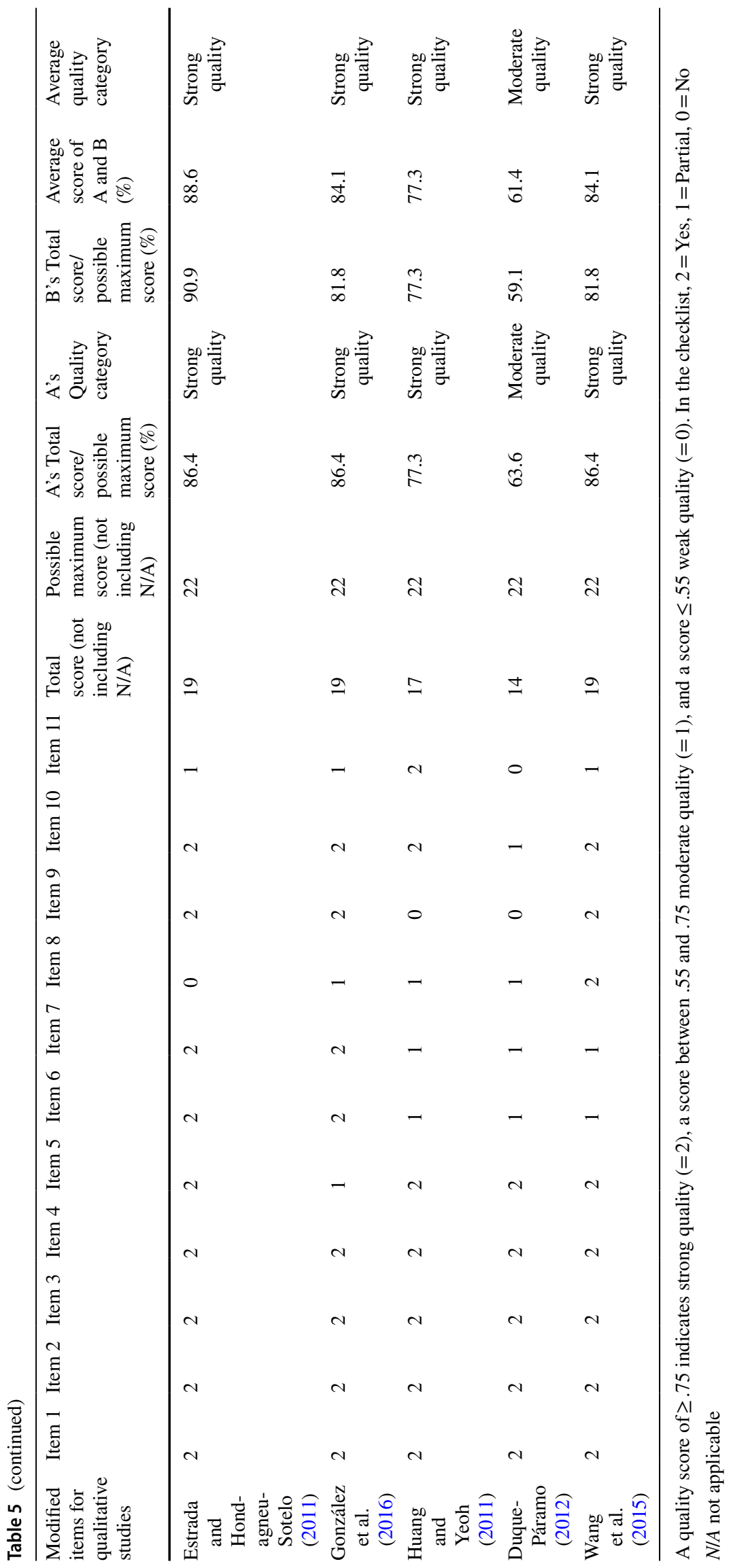




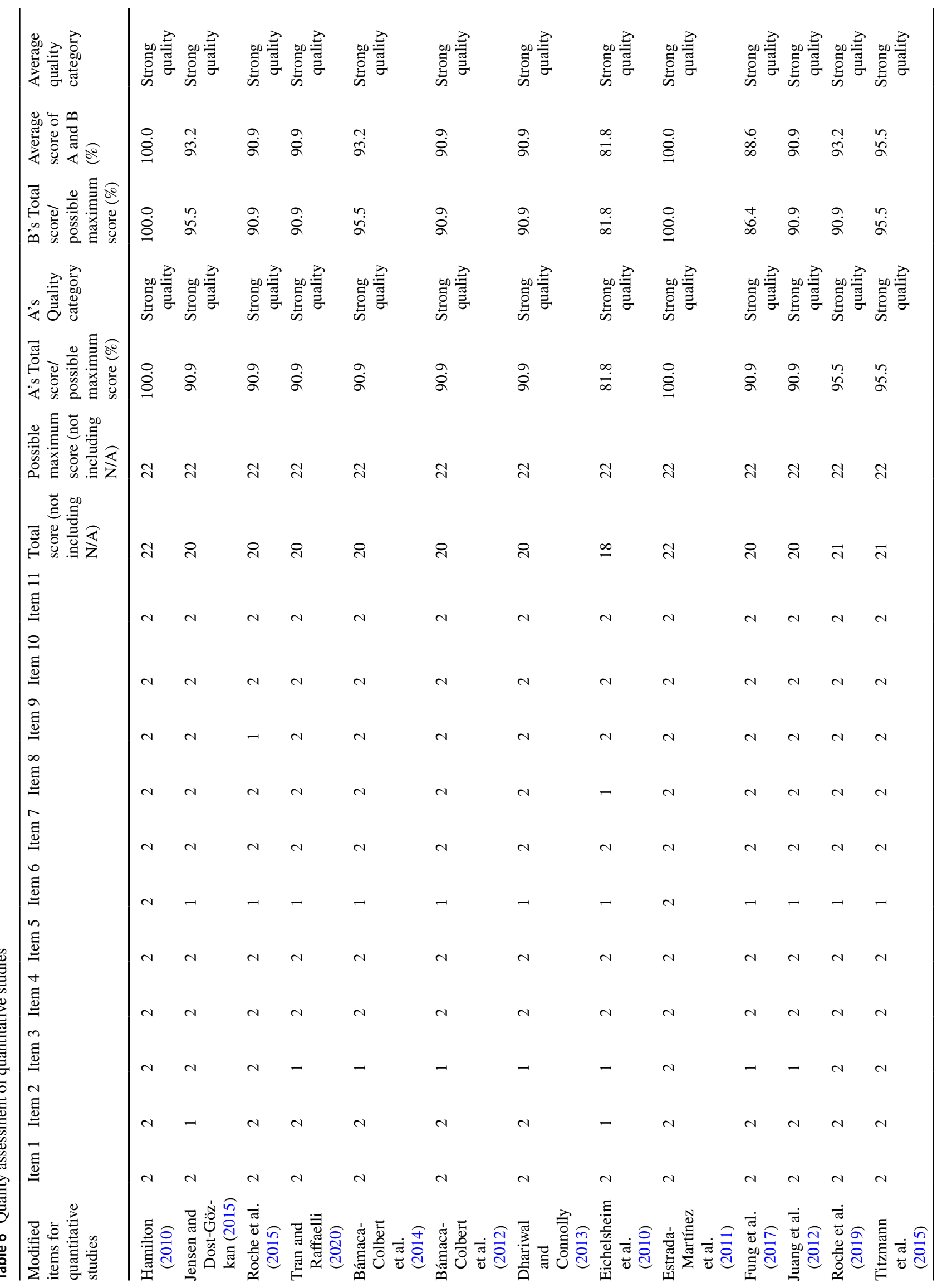




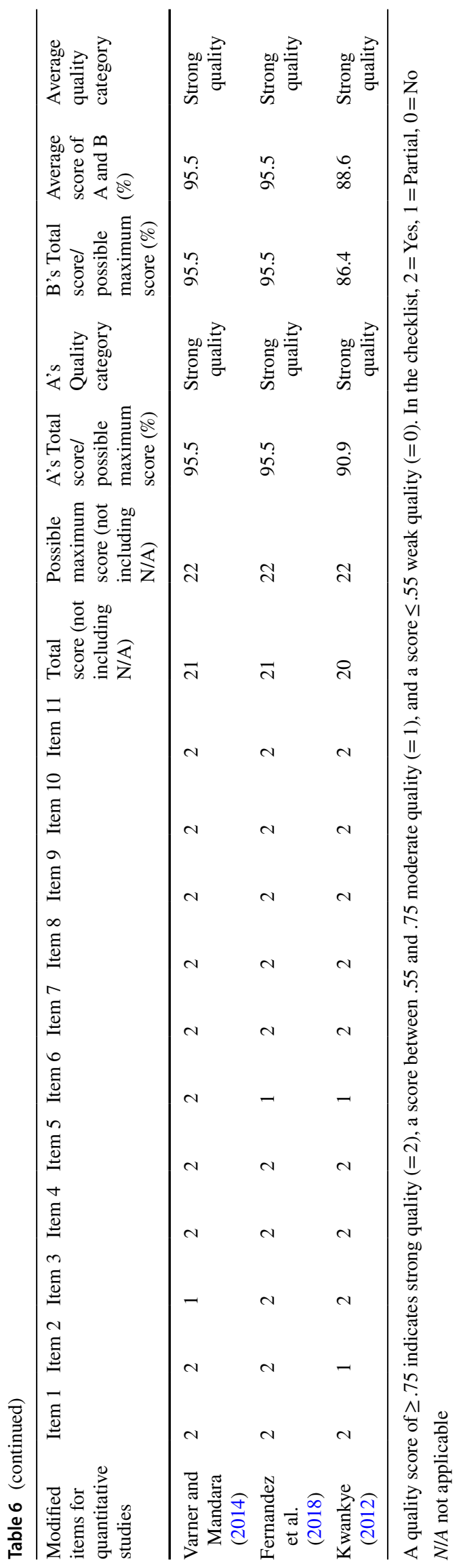

Springer 


$$
\text { L }
$$




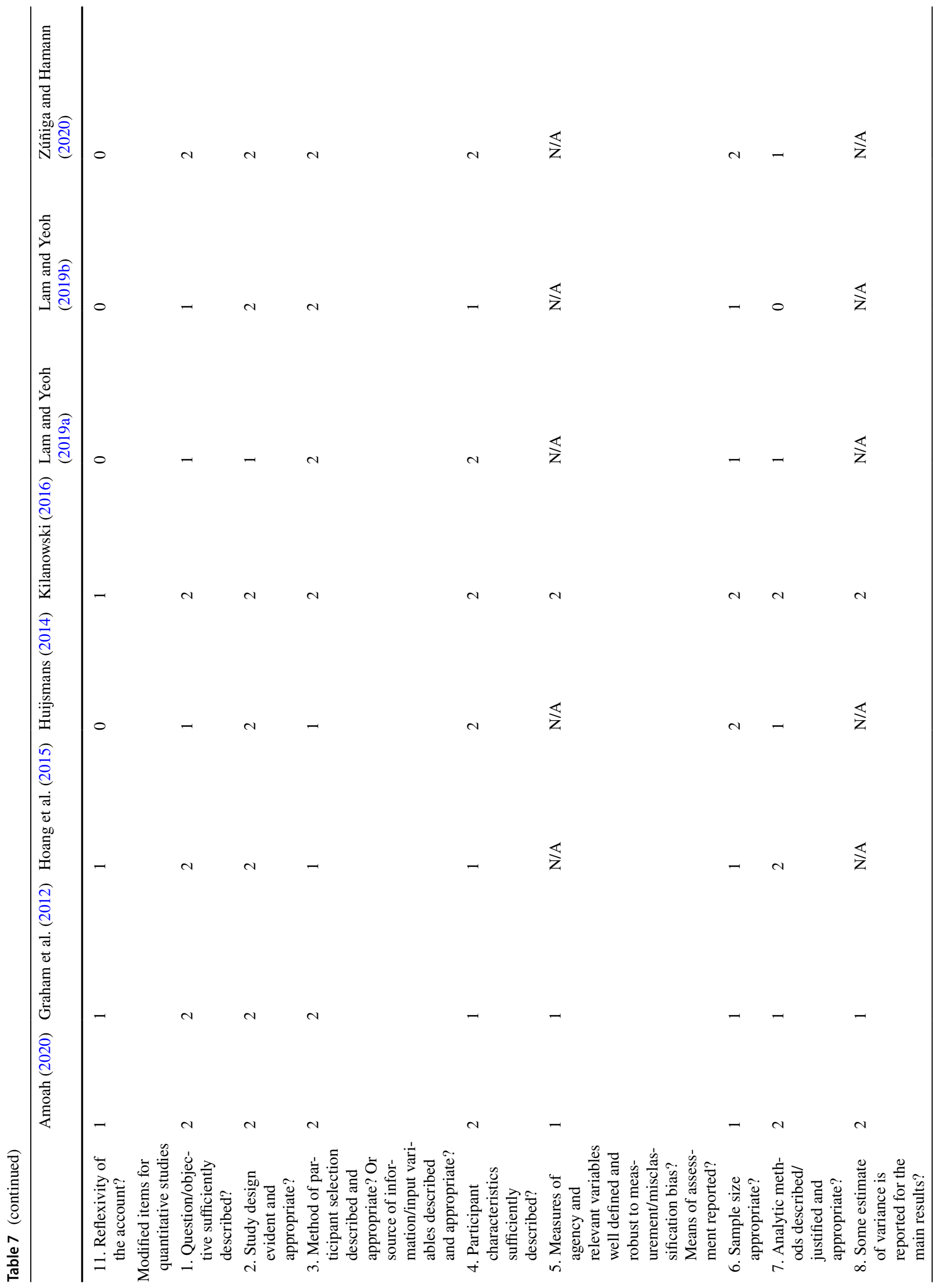




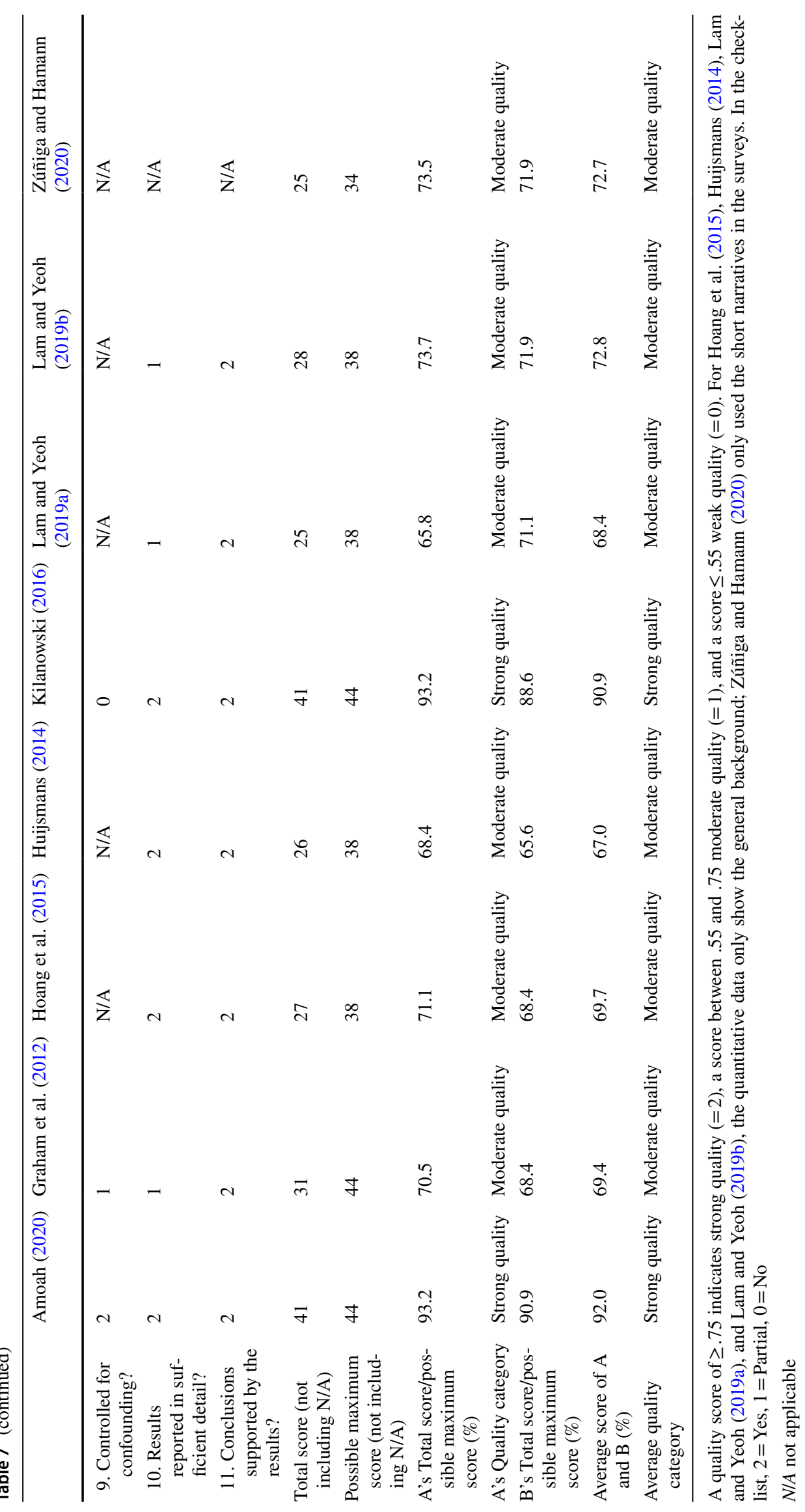


Supplementary Information The online version contains supplementary material available at https://doi.org/10.1007/s40894-021-00175-0.

Acknowledgements The authors would like to express their gratitude to the Editor of Adolescent Research Review, Roger J. R. Levesque, who provided valuable comments on the article. The authors are also grateful to UNSW Library Research Consultation for their advice on conducting systematic reviews.

Author Contributions ZD conceived of the study, designed the review, conducted literature searches, data screening and extraction, quality assessment and formal analysis, wrote the original draft of the manuscript, and reviewed and edited the manuscript; JX participated in the data screening and extraction, conducted quality assessment, and reviewed and edited the manuscript; IK and BL participated in designing the review and data screening, reviewed and edited the manuscript, and provided supervision. All authors read and approved the final manuscript.

Funding The authors did not receive support from any organization for the submitted work

\section{Declarations}

Conflict of interest The authors report no conflict of interests.

\section{Preregistration None.}

Ethical Approval This study has used the existing literature to do the systematic review. The data have no individually identifiable information, and thus this research does not go through a full review by the Ethics Committee.

Informed Consent Using the existing literature, informed consent is not included in this research. The authors do not know who the participants are and do not have access to the participants' contact information.

\section{References}

\section{* References marked with an asterisk indicate studies included in the systematic review}

Abebe, T. (2019). Reconceptualising children's agency as continuum and interdependence. Social Sciences. https://doi.org/10.3390/ socsci8030081

Abrego, L. J. (2014). Sacrificing families: Navigating laws, labor, and love across borders. Stanford University Press.

* Adefehinti, B., \& Arts, K. (2019). Challenging the odds of vulnerability and resilience in lone migration: Coping strategies of Zimbabwean unaccompanied minors in South Africa. Children's Geographies, 17(4), 427-441. https://doi.org/10.1080/14733285. 2018.1536776

Ahearn, L. M. (1999). Agency. Journal of Linguistic Anthropology, 9(1-2), 12-15. https://doi.org/10.1525/jlin.1999.9.1-2.12

* Akinsulure-Smith, A. M., Mirpuri, S., Chu, T., Keatley, E., \& Rasmussen, A. (2016). Made in America: Perspectives on friendship in West African immigrant families. Journal of Child and Family Studies, 25(9), 2765-2777. https://doi.org/10.1007/ s10826-016-0431-8
Alanen, L. (2001). Childhood as a generational condition: Children's daily lives in a central Finland town. In L. Alanen \& B. Mayall (Eds.), Conceptualizing child-adult relations (pp. 129-143). Routledge Falmer.

* Amoah, P. A. (2020). Perceptions of neglect and well-being among independent child migrants in Ghana. Child Indicators Research, 13(2), 455-479. https://doi.org/10.1007/s12187-019-09678-8

* Ashbourne, L. M., \& Baobaid, M. (2014). Parent-adolescent storytelling in Canadian-Arabic immigrant families (Part 2): A narrative analysis of adolescents' stories told to parents. The Qualitative Report, 19(30), 1-18. https://doi.org/10.46743/2160-3715/ 2014.1034

Asis, M. M. B. (2006). Living with migration: Experiences of leftbehind children in the Philippines. Asian Population Studies, 2(1), 45-67.

* Bakken, J. P., \& Brown, B. B. (2010). Adolescent secretive behavior: African American and Hmong adolescents' strategies and justifications for managing parents' knowledge about peers. Journal of Research on Adolescence, 20(2), 359-388. https://doi.org/10. 1111/j.1532-7795.2010.00642.x

* Bámaca-Colbert, M. Y., Greene, K. M., Killoren, S. E., \& Noah, A. J. (2014). Contextual and developmental predictors of sexual initiation timing among Mexican-origin girls. Developmental Psychology, 50(10), 2353-2359. https://doi.org/10.1037/a0037772

* Bámaca-Colbert, M. Y., Umaña-Taylor, A. J., \& Gayles, J. G. (2012). A developmental-contextual model of depressive symptoms in Mexican-origin female adolescents. Developmental Psychology, 48(2), 406-421. https://doi.org/10.1037/a0025666

Bluebond-Langner, M., \& Korbin, J. E. (2007). Challenges and opportunities in the anthropology of childhoods: An introduction to "Children, Childhoods, and Childhood Studies." American Anthropologist, 109(2), 241-246.

Bordonaro, L. I. (2012). Agency does not mean freedom. Cape Verdean street children and the politics of children's agency. Children's Geographies, 10(4), 413-426.

* Caneva, E. (2015). Children's agency and migration: Constructing kinship in Latin American and East European families living in Italy. Childhood, 22(2), 278-292. https://doi.org/10.1177/09075 68214521844

* Cense, M. (2014). Sexual discourses and strategies among minority ethnic youth in the Netherlands. Culture, Health and Sexuality, 16(7), 835-849. https://doi.org/10.1080/13691058.2014.918655

Choi, S. Y. P., Yeoh, B. S. A., \& Lam, T. (2019). Editorial introduction: Situated agency in the context of research on children, migration, and family in Asia. Population, Space and Place, 25(3), e2149.

* Clark, L. S., \& Sywyj, L. (2012). Mobile intimacies in the USA among refugee and recent immigrant teens and their parents. Feminist Media Studies, 12(4), 485-495. https://doi.org/10.1080/ 14680777.2012.741861

* Clayton, C. L. (2013). British Chinese children: Agency and action. The Journal of Early Adolescence, 33(2), 161-183. https://doi. org/10.1177/0272431611429946

Cummings, E. M., \& Schermerhorn, A. C. (2003). A developmental perspective on children as agents in the family. In L. Kuczynski (Ed.), Handbook of dynamics in parent-child relations (pp. 91-108). SAGE Publications, Inc. https://doi.org/10.4135/97814 52229645.n5.

* Dhariwal, A., \& Connolly, J. (2013). Romantic experiences of homeland and diaspora South Asian youth: Westernizing processes of media and friends. Journal of Research on Adolescence, 23(1), 45-56. https://doi.org/10.1111/j.1532-7795.2012.00803.x

Dreby, J. (2010). Divided by borders: Mexican migrants and their children. University of California Press.

* Duque-Páramo, M. C. (2012). Parental migration in Colombia: Children's voices in national and international perspectives. The 
Journal of Latin American and Caribbean Anthropology, 17(3), 472-492. https://doi.org/10.1111/j.1935-4940.2012.01251.x

Durham, D. (2008). Apathy and agency: The romance of agency and youth in Botswana. Figuring the Future: Globalization and the Temporalities of Children and Youth, 151-178.

* Edinburgh, L. D., Garcia, C. M., \& Saewyc, E. M. (2013). It's called "Going out to play": A video diary study of Hmong girls' perspectives on running away. Health Care for Women International, 34(2), 150-168. https://doi.org/10.1080/07399332.2011. 645962

* Eichelsheim, V. I., Buist, K. L., Deković, M., Wissink, I. B., Frijns, T., van Lier, P. A. C., et al. (2010). Associations among the parent-adolescent relationship, aggression and delinquency in different ethnic groups: A replication across two Dutch samples. Social Psychiatry and Psychiatric Epidemiology, 45(3), 293300. https://doi.org/10.1007/s00127-009-0071-z

* Estrada-Martínez, L. M., Padilla, M. B., Caldwell, C. H., \& Schulz, A. J. (2011). Examining the influence of family environments on youth violence: A comparison of Mexican, Puerto Rican, Cuban, Non-Latino Black, and Non-Latino White adolescents. Journal of Youth and Adolescence, 40(8), 1039-1051. https://doi.org/10. 1007/s10964-010-9624-4

* Estrada, E. (2013). Changing household dynamics: Children's American generational resources in street vending markets. Childhood, 20(1), 51-65. https://doi.org/10.1177/0907568212458441

* Estrada, E., \& Hondagneu-Sotelo, P. (2011). Intersectional dignities: Latino immigrant street vendor youth in Los Angeles. Journal of Contemporary Ethnography, 40(1), 102-131. https://doi.org/10. $1177 / 0891241610387926$

Fattore, T., Mason, J., \& Watson, E. (2009). When children are asked about their well-being: Towards a framework for guiding policy. Child Indicators Research, 2(1), 57-77.

* Fernandez, A., Loukas, A., \& Pasch, K. E. (2018). Examining the bidirectional associations between adolescents' disclosure, parents' solicitation, and adjustment problems among non-Hispanic White and Hispanic early adolescents. Journal of Youth and Adolescence, 47(12), 2569-2583. https://doi.org/10.1007/ s10964-018-0896-4

* Fung, J., Kim, J. J., Jin, J., Wu, Q., Fang, C., \& Lau, A. S. (2017). Perceived social change, parental control, and family relations: A comparison of Chinese families in Hong Kong, Mainland China, and the United States. Frontiers in Psychology, 8, 1671. https:// doi.org/10.3389/fpsyg.2017.01671

Gale, N. K., Heath, G., Cameron, E., Rashid, S., \& Redwood, S. (2013). Using the framework method for the analysis of qualitative data in multi-disciplinary health research. BMC Medical Research Methodology, 13(1), 1-8.

* Giuliani, C., Olivari, M. G., \& Alfieri, S. (2017). Being a "Good" son and a "Good" daughter: Voices of Muslim immigrant adolescents. Social Sciences. https://doi.org/10.3390/socsci6040142

* Glozman, J., \& Chuang, S. S. (2019). Multidimensional acculturation and identity of Russian-speaking youth in Canada: The role of parents. Journal of Adolescent Research, 34(4), 464-488. https:// doi.org/10.1177/0743558418767624

Goh, E. C. L., \& Kuczynski, L. (2009). Agency and power of single children in multi-generational families in urban Xiamen, China. Culture and Psychology, 15(4), 506-532. https://doi.org/10. 1177/1354067X09344881

* González, B. R., Cantú, E. C., \& Hernández-León, R. (2016). Moving to the 'Homeland': Children's narratives of migration from the United States to Mexico. Mexican Studies, 32(2), 252-275. https://doi.org/10.1525/mex.2016.32.2.252

* Graham, E., Jordan, L. P., Yeoh, B. S. A., Lam, T., Asis, M., \& SuKamdi. . (2012). Transnational families and the family nexus: Perspectives of Indonesian and Filipino children left behind by migrant parent(s). Environment and Planning a: Economy and Space, 44(4), 793-815. https://doi.org/10.1068/a4445

* Hamilton, H. A. (2010). Adolescent independence across immigrant generations: Age and ethnic variations. Sociological Focus, 43(3), 240-258. https://doi.org/10.1080/00380237.2010.10571 378

Hardman, C. (2001). Can there be an Anthropology of Children? Childhood, 8(4), 501-517. https://doi.org/10.1177/090756820100800 4006

* He, A. W. (2016). Discursive roles and responsibilities: A study of interactions in Chinese immigrant households. Journal of Multilingual and Multicultural Development, 37(7), 667-679. https:// doi.org/10.1080/01434632.2015.1127930

Hernandez, M., \& Iyengar, S. S. (2001). What drives whom? A cultural perspective on human agency. Social Cognition, 19(3), 269-294. https://doi.org/10.1521/soco.19.3.269.21468

* Hoang, L. A., Lam, T., Yeoh, B. S. A., \& Graham, E. (2015). Transnational migration, changing care arrangements and left-behind children's responses in South-east Asia. Children's Geographies, 13(3), 263-277. https://doi.org/10.1080/14733285.2015.972653

* Hoang, L. A., \& Yeoh, B. S. A. (2015). Children's agency and its contradictions in the context of transnational labour migration from Vietnam. Global Networks, 15(2), 180-197. https://doi.org/ 10.1111/glob. 12057

Hoffman, D. M. (2010). Migrant children in Haiti: Domestic labor and the politics of representation. In M. O. Ensor \& E. M. Goździak (Eds.), Children and migration: At the crossroads of resiliency and vulnerability (pp. 36-53). Palgrave Macmillan. https://doi. org/10.1057/9780230297098_3.

* Huang, S., \& Yeoh, B. S. A. (2011). Navigating the terrains of transnational education: Children of Chinese 'study mothers' in Singapore. Geoforum, 42(3), 394-403. https://doi.org/10.1016/j. geoforum.2011.01.010

Huijsmans, R. (2011). Child migration and questions of agency. Development and Change, 42(5), 1307-1321. https://doi.org/10.1111/j. 1467-7660.2011.01729.x

* Huijsmans, R. (2014). Becoming a young migrant or stayer seen through the lens of 'householding': Households 'in flux' and the intersection of relations of gender and seniority. Geoforum, 51, 294-304. https://doi.org/10.1016/j.geoforum.2012.11.007

* Hutchins, T. (2011). 'They Told Us in a Curry Shop': Child-adult relations in the context of family migration decision-making. Journal of Ethnic and Migration Studies, 37(8), 1219-1235. https://doi.org/10.1080/1369183X.2011.590926

James, A., \& James, A. (2004). Constructing childhood: Theory, policy and social practice. Palgrave Macmillan.

Jeffrey, C. (2011). Geographies of children and youth II: Global youth agency. Progress in Human Geography, 36(2), 245-253. https:// doi.org/10.1177/0309132510393316

* Jensen, L. A., \& Dost-Gözkan, A. (2015). Adolescent-parent relations in Asian Indian and Salvadoran immigrant families: A cultural-developmental analysis of autonomy, authority, conflict, and cohesion. Journal of Research on Adolescence, 25(2), 340-351. https://doi.org/10.1111/jora.12116

* Juang, L. P., Syed, M., \& Cookston, J. T. (2012). Acculturation-based and everyday parent-adolescent conflict among Chinese American adolescents: Longitudinal trajectories and implications for mental health. Journal of Family Psychology, 26(6), 916-926. https://doi.org/10.1037/a0030057

Kämpfe, K., \& Westphal, M. (2016). Acquiring agency: Children's perspective within the context of migration in Germany. In C. Hunner-Kreisel \& S. Bohne (Eds.), Childhood, youth and migration (pp. 63-80). Springer.

Kapadia, S., \& Miller, J. (2005). Parent-adolescent relationships in the context of interpersonal disagreements: View from a collectivist 
culture. Psychology and Developing Societies, 17(1), 33-50. https://doi.org/10.1177/097133360501700103

Katz, E. (2015). Domestic violence, children's agency and motherchild relationships: Towards a more advanced model. Children and Society, 29(1), 69-79.

* Katz, V. S. (2010). How children of immigrants use media to connect their families to the community. Journal of Children and Media, 4(3), 298-315. https://doi.org/10.1080/17482798.2010.486136

* Kilanowski, J. F. (2016). Influences on healthy-eating decision making in Latino adolescent children of migrant and seasonal agricultural workers. Journal of Pediatric Health Care, 30(3), 224-230. https://doi.org/10.1016/j.pedhc.2015.07.004

Kmet, L. M., Cook, L. S., \& Lee, R. C. (2004). Standard quality assessment criteria for evaluating primary research papers from a variety of fields (HTA Initia.). Alberta Heritage Foundation for Medical Research (AHFMR).

Kuczynski, L. (2003). Beyond bidirectionality: Bilateral conceptual frameworks for understanding dynamics in parent-child relations. In L. Kuczynski (Ed.), Handbook of dynamics in parentchild relations (pp. 3-24). SAGE Publications, Inc. https://doi. org/10.4135/9781452229645.n1.

* Kwankye, S. O. (2012). Independent north-south child migration as a parental investment in northern Ghana. Population, Space and Place, 18(5), 535-550. https://doi.org/10.1002/psp.682

* Lagomarsino, F., \& Castellani, S. (2016). The unseen protagonists. Ecuadorians' daughters between Ecuador and Southern Europe. Social Identities, 22(3), 291-306. https://doi.org/10.1080/13504 630.2015 .1128813

* Lalander, P., \& Herz, M. (2018). 'I Am Going to Europe Tomorrow': The myth of the anchor child and the decision to flee in the narratives of unaccompanied children. Nordic Journal of Migration Research, 8(2), 91-98. https://doi.org/10.1515/njmr-2018-0001

* Lam, T., \& Yeoh, B. S. A. (2019a). Parental migration and disruptions in everyday life: Reactions of left-behind children in Southeast Asia. Journal of Ethnic and Migration Studies, 45(16), 3085-3104. https://doi.org/10.1080/1369183X.2018.1547022

* Lam, T., \& Yeoh, B. S. A. (2019b). Under one roof? Left-behind children's perspectives in negotiating relationships with absent and return-migrant parents. Population, Space and Place, 25(3), e2151. https://doi.org/10.1002/psp.2151

Landais, L. L., Damman, O. C., Schoonmade, L. J., Timmermans, D. R. M., Verhagen, E. A. L. M., \& Jelsma, J. G. M. (2020). Choice architecture interventions to change physical activity and sedentary behavior: A systematic review of effects on intention, behavior and health outcomes during and after intervention. International Journal of Behavioral Nutrition and Physical Activity, 17(1), 47. https://doi.org/10.1186/s12966-020-00942-7

* Lee, H. (2016). 'I was forced here': Perceptions of agency in second generation 'return' migration to Tonga. Journal of Ethnic and Migration Studies, 42(15), 2573-2588. https://doi.org/10.1080/ 1369183X.2016.1176524

Lee, J. L. C., Lo, T. L. T., \& Ho, R. T. H. (2018). Understanding outdoor gyms in public open spaces: A systematic review and integrative synthesis of qualitative and quantitative evidence. International Journal of Environmental Research and Public Health, 15(4), 590

* Li, J. (2010). 'My home and my school': Examining immigrant adolescent narratives from the critical sociocultural perspective. Race Ethnicity and Education, 13(1), 119-137. https://doi.org/ 10.1080/13613320903550154

Liberati, A., Altman, D. G., Tetzlaff, J., Mulrow, C., Gøtzsche, P. C., Ioannidis, J. P. A., et al. (2009). The PRISMA statement for reporting systematic reviews and meta-analyses of studies that evaluate healthcare interventions: Explanation and elaboration. $B M J, 339$, b2700. https://doi.org/10.1136/bmj.b2700
Luescher, K., \& Pillemer, K. (1998). Intergenerational ambivalence: A new approach to the study of parent-child relations in later life. Journal of Marriage and Family, 60(2), 413-425. https:// doi.org/10.2307/353858

Mahmood, S. (2001). Feminist theory, embodiment, and the docile agent: Some reflections on the Egyptian Islamic revival. Cultural Anthropology, 16(2), 202-236. https://doi.org/10.1525/can.2001. 16.2.202

Mannion, G. (2007). Going Spatial, Going Relational: Why "listening to children" and children's participation needs reframing. Discourse: Studies in the Cultural Politics of Education, 28(3), 405-420. https://doi.org/10.1080/01596300701458970

* Mizen, P., \& Ofosu-Kusi, Y. (2013). Agency as vulnerability: Accounting for children's movement to the streets of Accra. The Sociological Review, 61(2), 363-382. https://doi.org/10.1111/ 1467-954X.12021

* Moskal, M., \& Tyrrell, N. (2016). Family migration decision-making, step-migration and separation: Children's experiences in European migrant worker families. Children's Geographies, 14(4), 453-467. https://doi.org/10.1080/14733285.2015.1116683

* Nazridod, S., da Pereira, C. P., \& C., \& Guerreiro, M. das D. H. . (2019). Adolescents who stay, parents who migrate: Gender inequalities, resilience and coping strategies in Tajikistan. Journal of Ethnic and Migration Studies, . https://doi.org/10.1080/ 1369183X.2019.1662716

Orellana, M. F., Thorne, B., Chee, A., \& Lam, W. S. E. (2001). Transnational childhoods: The participation of children in processes of family migration. Social Problems, 48(4), 572-591.

* Pang, B., Macdonald, D., \& Hay, P. (2015). 'Do I have a choice?' The influences of family values and investments on Chinese migrant young people's lifestyles and physical activity participation in Australia. Sport, Education and Society, 20(8), 1048-1064. https://doi.org/10.1080/13573322.2013.833504

* Pantea, M.-C. (2011). Young people's perspectives on changing families' dynamics of power in the context of parental migration. Young, 19(4), 375-395. https://doi.org/10.1177/1103308811 01900402

* Pantea, M.-C. (2012). 'I have a child and a garden': Young people's experiences of care giving in transnational families. Journal of Youth Studies, 15(2), 241-256. https://doi.org/10.1080/13676 261.2011.634399

* Peltola, M., Keskinen, S., Honkasalo, V., \& Honkatukia, P. (2017). Intergenerational negotiations on (hetero)sexuality and romantic relationships-Views of young people and parents in multi-ethnic contexts. Journal of Youth Studies, 20(5), 533-548. https:// doi.org/10.1080/13676261.2016.1241870

Phạm, Q. N. (2013). Enduring bonds: Politics and life outside freedom as autonomy. Alternatives: Global, Local, Political, 38(1), 29-48. https://doi.org/10.2307/23412518

Punch, S. (2007). Negotiating migrant identities: Young people in Bolivia and Argentina. Children's Geographies, 5(1-2), 95-112. https://doi.org/10.1080/14733280601108213

Reardon, T., Harvey, K., Baranowska, M., O’Brien, D., Smith, L., \& Creswell, C. (2017). What do parents perceive are the barriers and facilitators to accessing psychological treatment for mental health problems in children and adolescents? A systematic review of qualitative and quantitative studies. European Child and Adolescent Psychiatry, 26(6), 623-647.

* Roche, K. M., Lambert, S. F., Ghazarian, S. R., \& Little, T. D. (2015). Adolescent language brokering in diverse contexts: Associations with parenting and parent-youth relationships in a new immigrant destination area. Journal of Youth and Adolescence, 44(1), 77-89. https://doi.org/10.1007/s10964-014-0154-3

* Roche, K. M., Lambert, S. F., White, R. M. B., Calzada, E. J., Little, T. D., Kuperminc, G. P., \& Schulenberg, J. E. (2019). Autonomyrelated parenting processes and adolescent adjustment in Latinx 
immigrant families. Journal of Youth and Adolescence, 48(6), 1161-1174. https://doi.org/10.1007/s10964-019-01010-5

* Romo, L. F., Mireles-Rios, R., \& Lopez-Tello, G. (2014). Latina mothers' and daughters' expectations for autonomy at age 15 (La Quinceañera). Journal of Adolescent Research, 29(2), 271-294. https://doi.org/10.1177/0743558413477199

Rübner Jørgensen, C. (2016). 'The problem is that I don't know'Agency and life projects of transnational migrant children and young people in England and Spain. Childhood, 24(1), 21-35. https://doi.org/10.1177/0907568216646567

* Saint-Blancat, C. M., \& Zaltron, F. (2013). 'Making the most of it ...': How young Romanians and Moroccans in north-eastern Italy use resources from their social networks. Ethnicities, 13(6), 795-817. https://doi.org/10.1177/1468796812472148

Sarausad, M. R. G. A. (2006). Struggles from within: Migrant women in Southeast Asia. Development, 49(1), 134-136. https://doi.org/ 10.1057/palgrave.development.1100217

* Sime, D., \& Fox, R. (2015a). Migrant children, social capital and access to services post-migration: Transitions, negotiations and complex agencies. Children and Society, 29(6), 524-534. https:// doi.org/10.1111/chso.12092

* Sime, D., \& Fox, R. (2015b). Home abroad: Eastern European children's family and peer relationships after migration. Childhood, 22(3), 377-393. https://doi.org/10.1177/0907568214543199

* Sime, D., \& Pietka-Nykaza, E. (2015). Transnational intergenerationalities: Cultural learning in Polish migrant families and its implications for pedagogy. Language and Intercultural Communication, 15(2), 208-223. https://doi.org/10.1080/14708477. 2014.993324

* Stuart, J., Ward, C., Jose, P. E., \& Narayanan, P. (2010). Working with and for communities: A collaborative study of harmony and conflict in well-functioning, acculturating families. International Journal of Intercultural Relations, 34(2), 114-126. https://doi. org/10.1016/j.ijintrel.2009.11.004

Sutterlüty, F., \& Tisdall, E. K. M. (2019). Agency, autonomy and selfdetermination: Questioning key concepts of childhood studies. Global Studies of Childhood, 9(3), 183-187. https://doi.org/10. 1177/2043610619860992

* Tanyas, B. (2012). Making sense of migration: Young Turks' experiences in the United Kingdom. Journal of Youth Studies, 15(6), 693-710. https://doi.org/10.1080/13676261.2012.671931

* Titzmann, P. F., Gniewosz, B., \& Michel, A. (2015). Two sides of a story: Mothers' and adolescents' agreement on child disclosure in immigrant and native families. Journal of Youth and Adolescence, 44(1), 155-169. https://doi.org/10.1007/ s10964-013-0077-4

Tong, A., Flemming, K., McInnes, E., Oliver, S., \& Craig, J. (2012). Enhancing transparency in reporting the synthesis of qualitative research: ENTREQ. BMC Medical Research Methodology, 12(1), 181. https://doi.org/10.1186/1471-2288-12-181

* Tran, S. P., \& Raffaelli, M. (2020). Configurations of autonomy and relatedness in a multiethnic U.S. sample of parent-adolescent dyads. Journal of Research on Adolescence, 30(1), 203-218. https://doi.org/10.1111/jora.12517

* Turjanmaa, E., Alitolppa-Niitamo, A., \& Jasinskaja-Lahti, I. (2017). 1.5-Generation immigrant adolescents' autonomy negotiations in transnational family contexts. Migration Letters, 14(1), 75-87. https://doi.org/10.33182/ml.v14i1.317

UNDESA. (2020). International Migrant Stock 2020. United Nations database. Retrieved September 12, 2021, from https://www.un. org/development/desa/pd/content/international-migrant-stock.

* Varner, F., \& Mandara, J. (2014). Differential parenting of African American adolescents as an explanation for gender disparities in achievement. Journal of Research on Adolescence, 24(4), 667-680. https://doi.org/10.1111/jora.12063

* Wang, Q., Koh, J. B. K., \& Song, Q. (2015). Meaning making through personal storytelling: Narrative research in the Asian American context. Asian American Journal of Psychology, 6(1), 88-96. https://doi.org/10.1037/a0037317

Xiao, L.-N., \& Yuan, Y. (2019). 儿童的能动性: 一个整合的解释框 架 [Children's agency: An integrated interpretive framework]. 学海 [Academia Bimestrie], (2), 72-78.

* Yau, J. (2016). Adolescent nondisclosure in cultural context: Voices of Chinese American adolescents and parents. Journal of Adolescent Research, 31(5), 606-630. https://doi.org/10.1177/07435 58415604218

* Zúñiga, V., \& Hamann, E. T. (2020). Children's voices about 'return' migration from the United States to Mexico: The 0.5 generation. Children's Geographies. https://doi.org/10.1080/14733285.2020. 1743818

Publisher's Note Springer Nature remains neutral with regard to jurisdictional claims in published maps and institutional affiliations. 